\title{
SUBTHRESHOLD CMOS LOGIC DESIGN USING PARALLEL TRANSISTOR STACKS
}

by

\author{
Manmit Muker, B.Eng. (Electrical) \\ Carleton University
}

\begin{abstract}
A thesis submitted to the Faculty of Graduate Studies and Research in partial fulfillment of the requirements for the degree of

Master of Applied Science
\end{abstract}

Ottawa-Carleton Institute for Electrical and Computer Engineering

Department of Electronics

Carleton University

Ottawa, Ontario, Canada

December 2010

(C) 2010 Manmit Muker 
Library and Archives
Canada

Published Heritage

Branch

395 Wellington Street

Ottawa ON K1A ON4

Canada
Bibliotheqque et

Archives Canada

Direction du

Patrimoine de l'édition

395, rue Wellington

Ottawa ON K1A ON4

Canada
Your file Votre référence

ISBN. 978-0-494-79544-6

Our file Notre référence

ISBN: 978-0-494-79544-6
NOTICE:

The author has granted a nonexclusive license allowing Library and Archives Canada to reproduce, publish, archive, preserve, conserve, communicate to the public by telecommunication or on the Internet, loan, distribute and sell theses worldwide, for commercial or noncommercial purposes, in microform, paper, electronic and/or any other formats.

The author retains copyright ownership and moral rights in this thesis. Neither the thesis nor substantial extracts from it may be printed or otherwise reproduced without the author's permission.
AVIS:

L'auteur a accordé une licence non exclusive permettant à la Bibliothèque et Archives Canada de reproduire, publier, archiver, sauvegarder, conserver, transmettre au public par télécommunication ou par l'Internet, prêter, distribuer et vendre des thèses partout dans le monde, à des fins commerciales ou autres, sur support microforme, papier, électronique et/ou autres formats.

L'auteur conserve la propriété du droit d'auteur et des droits moraux qui protège cette thèse. $\mathrm{Ni}$ la thèse ni des extraits substantiels de celle-ci ne doivent être imprimés ou autrement reproduits sans son autorisation.
In compliance with the Canadian Privacy Act some supporting forms may have been removed from this thesis.

While these forms may be included in the document page count, their removal does not represent any loss of content from the thesis.
Conformément à la loi canadienne sur la protection de la vie privée, quelques formulaires secondaires ont été enlevés de cette thèse.

Bien que ces formulaires aient inclus dans la pagination, il n'y aura aucun contenu manquant.

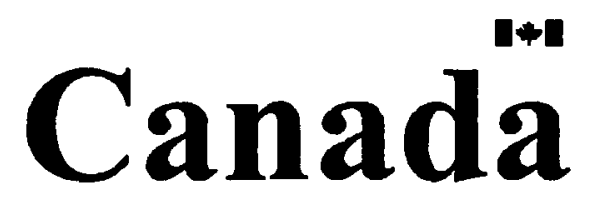




\begin{abstract}
The inverse-narrow-width effect in MOSFETs lowers the threshold voltage at narrower widths. The exponential dependence of the subthreshold current on the threshold voltage can result in higher than expected current at smaller widths. A design methodology that exploits this behavior is developed. A subthreshold optimum width that maximizes the current-to-capacitance ratio can be found. Using transistors of only this size either individually or in parallel stacks, as appropriate, leads to faster circuits in subthreshold. To design a CMOS digital logic circuit, a parallel transistor ratio is found that describes the ratio of PMOS-to-NMOS transistors to use. This is combined with the optimum subthreshold widths to determine an effective beta ratio, or effective PMOS-toNMOS width ratio, to use with sizing strategies such as Logical Effort. Delay reductions of up to $64.5 \%$ have been achieved in post-layout simulations with this design methodology compared to standard superthreshold designs.
\end{abstract}




\section{ACKNOWLEDGEMENTS}

I would like to thank

Prof. Maitham Shams for his guidance and enthusiasm.

Philip Tworzydlo for his assistance in performing certain simulations.

Jacques Lemieux and Scott Bruce for solving all my technical issues.

Professors John Knight and N. Garry Tarr for their careful review and contributions to the thesis.

CMC Microsystems and their technology partners for access to the design tools and technology kits used in the research for this thesis.

Prof. Pavan Gunupudi, Anna Lee and Blazenka Power for their help in bringing the thesis process to an end.

Jatinder and Jagmail Muker for their patience and support. 
TABLE OF CONTENTS

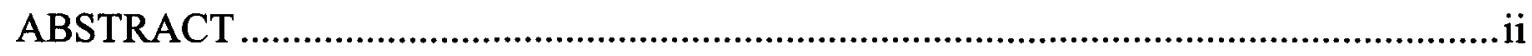

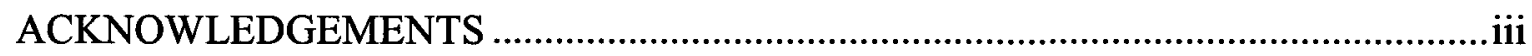

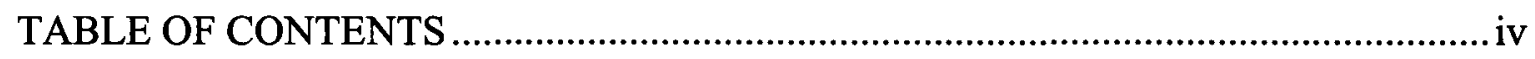

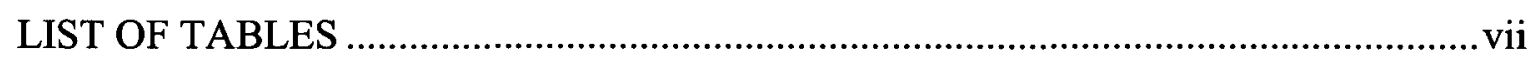

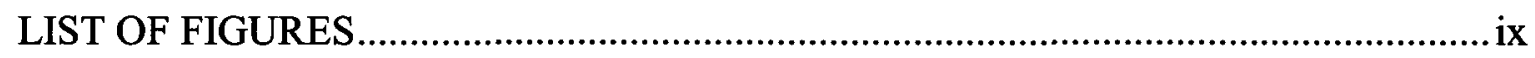

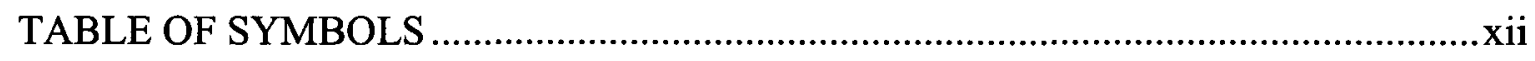

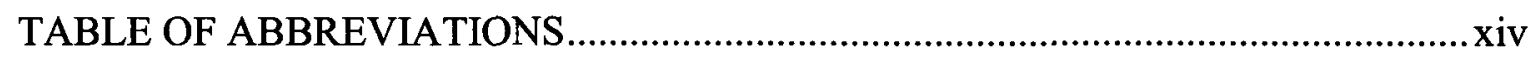

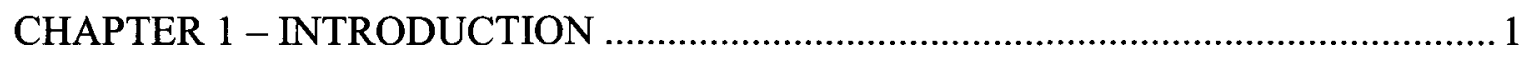

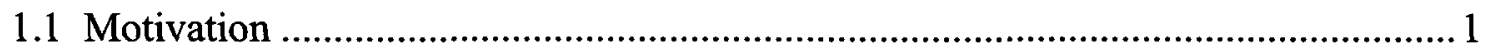

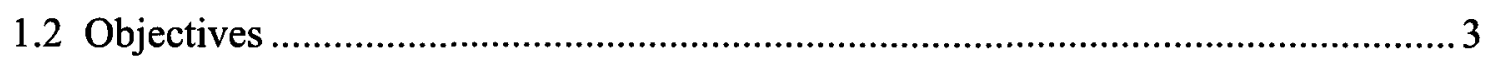

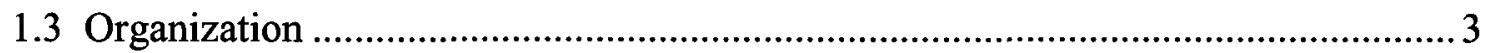

CHAPTER 2 - MOS TRANSISTOR PROPERTIES...................................................

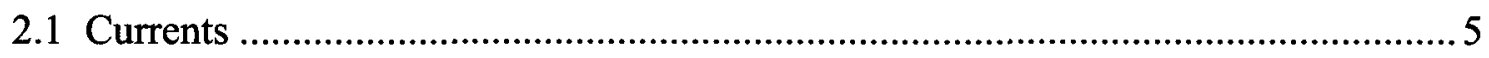

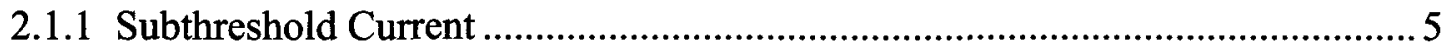

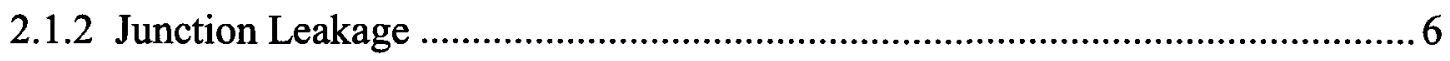

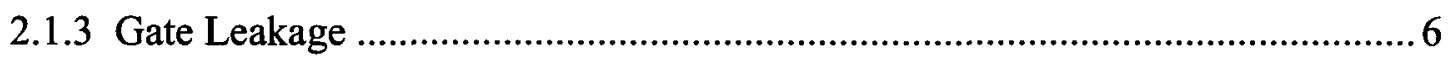

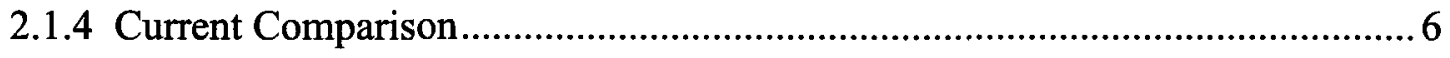

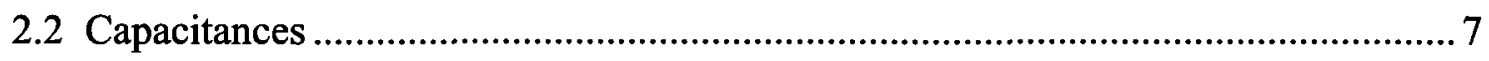




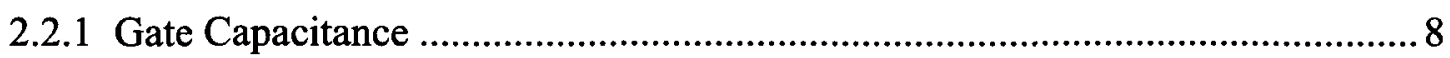

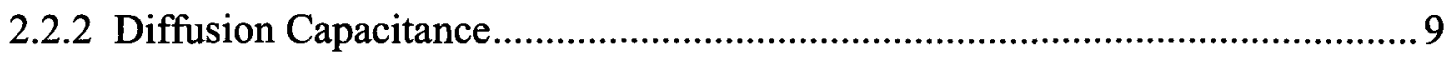

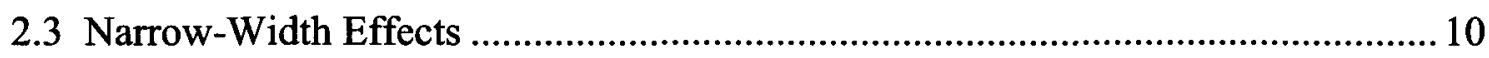

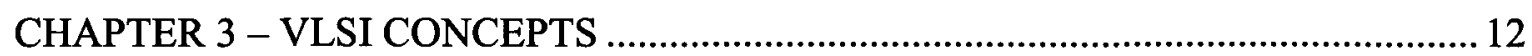

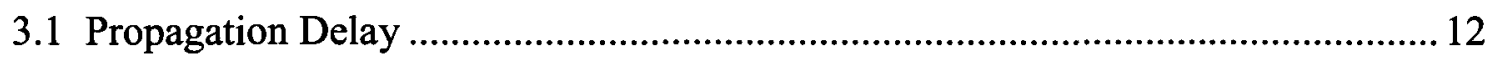

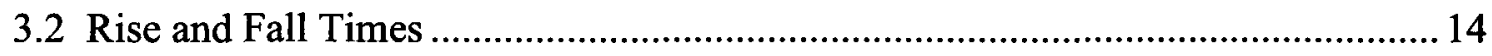

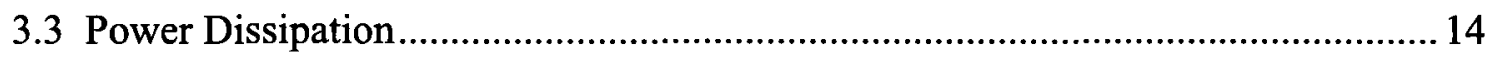

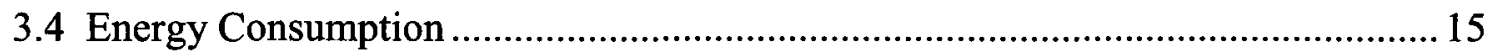

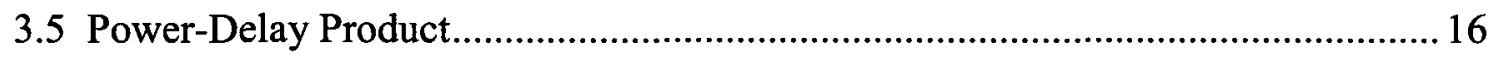

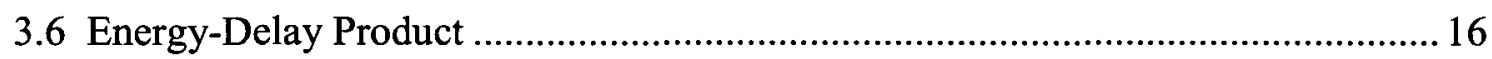

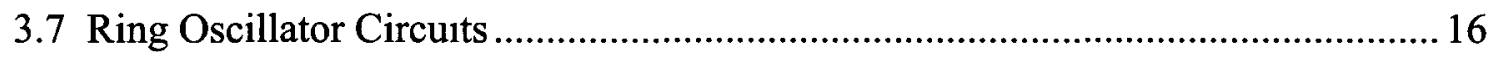

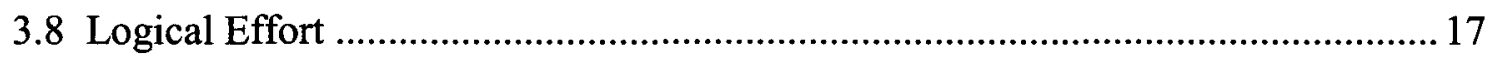

CHAPTER 4 - DESIGN METHODOLOGY DEVELOPMENT ................................... 19

4.1 Subthreshold Optimum Width from Current-to-Capacitance Ratio ........................ 19

4.2 Subthreshold Optimum Width from Ring Oscillator Circuit ................................... 31

4.3 Parallel Transistor Ratio .................................................................................. 43

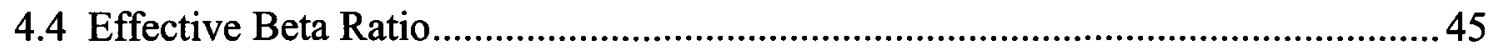

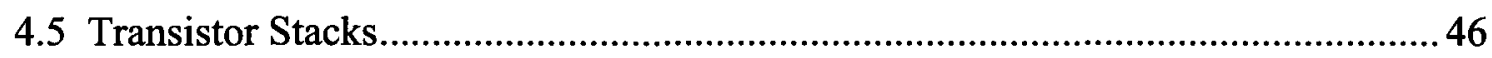

4.6 Ring Oscillator Tests for Parallel Transistor Stacks...............................................53

4.7 Subthreshold Logic Design Methodology …………...........................................5

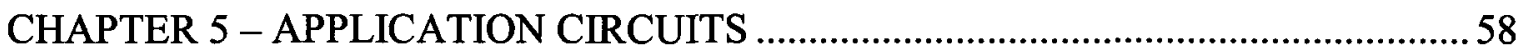




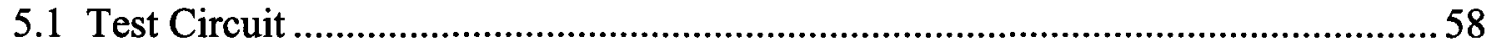

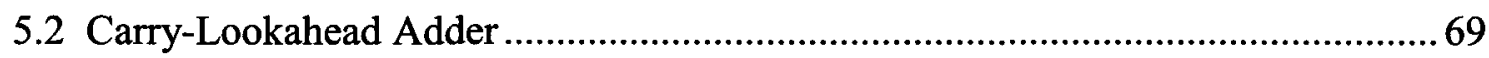

CHAPTER 6 - CONCLUSIONS AND FUTURE WORK …….....................................

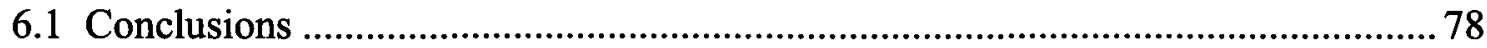

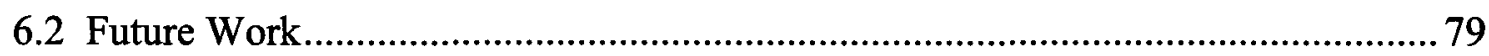

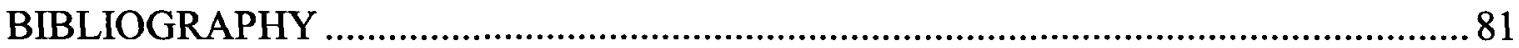




\section{LIST OF TABLES}

Table 1: $65 \mathrm{~nm}, V_{D D}=1 \mathrm{~V}$; currents for $0.12 \mu \mathrm{m}$ width transistors ...............................

Table 2: $65 \mathrm{~nm}, V_{D D}=0.3 \mathrm{~V}$; currents for $0.12 \mu \mathrm{m}$ width transistors ............................... 7

Table 3: $65 \mathrm{~nm} \mathrm{LP}, V_{D D}=1.2 \mathrm{~V}$; 9-inverter ring oscillator maximum frequencies for varying NMOS widths 34

Table 4: $65 \mathrm{~nm} \mathrm{LP}, V_{D D}=0.3 \mathrm{~V}$; 9-inverter ring oscillator maximum frequencies for varying NMOS widths 37

Table 5: $65 \mathrm{~nm} \mathrm{LP}, V_{D D}=0.3 \mathrm{~V}$; 9-inverter ring oscillator schematic and post-layout simulation frequencies 39

Table 6: $65 \mathrm{~nm}$ GP, $V_{D D}=1 \mathrm{~V}$; 9-inverter ring oscillator maximum frequencies for varying NMOS widths 40

Table 7: $65 \mathrm{~nm}$ GP, $V_{D D}=0.3 \mathrm{~V}$; 9-inverter ring oscillator maximum frequencies for varying NMOS widths 40

Table 8: $90 \mathrm{~nm}, V_{D D}=1.2 \mathrm{~V}$; 9-inverter ring oscillator maximum frequencies for varying NMOS widths. 41

Table 9: $90 \mathrm{~nm}, V_{D D}=0.3 \mathrm{~V}$; 9-inverter ring oscillator maximum frequencies for varying NMOS widths. 42

Table 10: Ring oscillator optimum widths. 42

Table 11: Subthreshold optimum widths, parallel transistor ratios and effective beta ratios

Table 12: $65 \mathrm{~nm} \mathrm{LP}, V_{D D}=1.2 \mathrm{~V}$; stack currents and scaled widths 48 
Table 13: $65 \mathrm{~nm} \mathrm{LP}, V_{D D}=0.3 \mathrm{~V}$; stack currents and scaled widths............................. 48

Table 14: $65 \mathrm{~nm} \mathrm{GP}, V_{D D}=1 \mathrm{~V}$; stack currents and scaled widths ................................. 49

Table 15: $65 \mathrm{~nm}$ GP, $V_{D D}=0.3 \mathrm{~V}$; stack currents and scaled widths ...............................49

Table 16: $90 \mathrm{~nm}, V_{D D}=1.2 \mathrm{~V}$; stack currents and scaled widths ..................................50

Table 17: $90 \mathrm{~nm}, V_{D D}=0.3 \mathrm{~V}$; stack currents and scaled widths ................................50

Table 18: $65 \mathrm{~nm} \mathrm{LP}, V_{D D}=0.3 \mathrm{~V}$; standard versus parallel configuration ring oscillator simulation results. .55

Table 19: $65 \mathrm{~nm}$ GP; parallel configuration with effective beta ratio under large load design example 62

Table 20: $65 \mathrm{~nm} \mathrm{LP}, V_{D D}=1.2 \mathrm{~V}$; test circuit propagation delays

Table 21: $65 \mathrm{~nm} \mathrm{LP}, V_{D D}=0.3 \mathrm{~V}$; test circuit propagation delays 64

Table 22: $65 \mathrm{~nm} \mathrm{GP}, V_{D D}=1 \mathrm{~V}$; test circuit propagation delays .65

Table 23: $65 \mathrm{~nm} \mathrm{GP}, V_{D D}=0.3 \mathrm{~V}$; test circuit propagation delays .66

Table 24: $90 \mathrm{~nm}, V_{D D}=1.2 \mathrm{~V}$; test circuit propagation delays 67

Table 25: $90 \mathrm{~nm}, V_{D D}=0.3 \mathrm{~V}$; test circuit propagation delays .68

Table 26: $65 \mathrm{~nm} \mathrm{LP}, V_{D D}=1.2 \mathrm{~V}$; carry-lookahead adder results (post-layout simulation results in brackets). .75

Table 27: $65 \mathrm{~nm} \mathrm{LP}, V_{D D}=0.3 \mathrm{~V}$; carry-lookahead adder results (post-layout simulation results in brackets). 


\section{LIST OF FIGURES}

Figure 1: Cross section of a MOSFET fabricated with (a) shallow trench isolation and (b) local oxidation of silicon

Figure 2: Transistor configuration for maximum current drive for (a) PMOS and (b) NMOS .20

Figure 3: $65 \mathrm{~nm} \mathrm{LP}, V_{D D}=1.2 \mathrm{~V}$; current $\left(I_{D}\right)$, threshold voltage $\left(V_{T}\right)$ and gate $\left(C_{G}\right)$ and source $\left(C_{S B}\right)$ diffusion capacitances versus width for (a) PMOS and (b) NMOS 20 Figure 4: $65 \mathrm{~nm} \mathrm{LP}, V_{D D}=0.3 \mathrm{~V}$; current $\left(I_{D}\right)$, threshold voltage $\left(V_{T}\right)$ and gate $\left(C_{G}\right)$ and source $\left(C_{S B}\right)$ diffusion capacitances versus width for (a) PMOS and (b) NMOS 21

Figure 5: $65 \mathrm{~nm} \mathrm{GP}, V_{D D}=1.0 \mathrm{~V}$; current $\left(I_{D}\right)$, threshold voltage $\left(V_{T}\right)$ and gate $\left(C_{G}\right)$ and source $\left(C_{S B}\right)$ diffusion capacitances versus width for (a) PMOS and (b) NMOS 22

Figure 6: $65 \mathrm{~nm} \mathrm{GP}, V_{D D}=0.3 \mathrm{~V}$; current $\left(I_{D}\right)$, threshold voltage $\left(V_{T}\right)$ and gate $\left(C_{G}\right)$ and source $\left(C_{S B}\right)$ diffusion capacitances versus width for (a) PMOS and (b) NMOS 23

Figure 7: $90 \mathrm{~nm}, V_{D D}=1.2 \mathrm{~V}$; current $\left(I_{D}\right)$, threshold voltage $\left(V_{T}\right)$ and gate $\left(C_{G}\right)$ and source $\left(C_{S B}\right)$ diffusion capacitances versus width for (a) PMOS and (b) NMOS 24

Figure 8: $90 \mathrm{~nm}, V_{D D}=0.3 \mathrm{~V}$; current $\left(I_{D}\right)$, threshold voltage $\left(V_{T}\right)$ and gate $\left(C_{G}\right)$ and source $\left(C_{S B}\right)$ diffusion capacitances versus width for (a) PMOS and (b) NMOS 25

Figure 9: $65 \mathrm{~nm} \mathrm{LP}, V_{D D}=1.2 \mathrm{~V}$; current-to-capacitance ratio $\left(I_{D} /\left(C_{G}+C_{S B}\right)\right)$ versus width for (a) PMOS and (b) NMOS 26

Figure 10: $65 \mathrm{~nm} \mathrm{LP}, V_{D D}=0.3 \mathrm{~V}$; current-to-capacitance ratio $\left(I_{D} /\left(C_{G}+C_{S B}\right)\right)$ versus width for (a) PMOS and (b) NMOS 27 
Figure 11: $65 \mathrm{~nm} \mathrm{GP}, V_{D D}=1.0 \mathrm{~V}$; current-to-capacitance ratio $\left(I_{D} /\left(C_{G}+C_{S B}\right)\right)$ versus width for (a) PMOS and (b) NMOS...

Figure 12: $65 \mathrm{~nm} \mathrm{GP}, V_{D D}=0.3 \mathrm{~V}$; current-to-capacitance ratio $\left(I_{D} /\left(C_{G}+C_{S B}\right)\right)$ versus width for (a) PMOS and (b) NMOS 29

Figure 13: $90 \mathrm{~nm}, V_{D D}=1.2 \mathrm{~V}$; current-to-capacitance ratio $\left(I_{D} /\left(C_{G}+C_{S B}\right)\right)$ versus width for (a) PMOS and (b) NMOS 30

Figure 14: $90 \mathrm{~nm}, V_{D D}=0.3 \mathrm{~V}$; current-to-capacitance ratio $\left(I_{D} /\left(C_{G}+C_{S B}\right)\right)$ versus width for (a) PMOS and (b) NMOS 31

Figure 15: $65 \mathrm{~nm} \mathrm{LP}, V_{D D}=1.2 \mathrm{~V}$; 9-inverter ring oscillator frequency versus PMOS width for $0.12 \mu \mathrm{m}$ NMOS width. 33

Figure 16: $65 \mathrm{~nm} \mathrm{LP}, \mathrm{V}_{\mathrm{DD}}=1.2 \mathrm{~V}$; 9-inverter ring osc1llator frequency as a function of PMOS and NMOS width 33

Figure 17: $65 \mathrm{~nm} \mathrm{LP}, V_{D D}=0.3 \mathrm{~V}$; 9-inverter ring oscillator frequency versus PMOS width for $0.12 \mu \mathrm{m}$ NMOS width .35

Figure 18: $65 \mathrm{~nm} \mathrm{LP}, V_{D D}=0.3 \mathrm{~V}$; 9-inverter ring oscillator frequency versus PMOS width for $1.08 \mu \mathrm{m}$ NMOS width. .36

Figure 19: $65 \mathrm{~nm} \mathrm{LP}, \mathrm{V}_{\mathrm{DD}}=0.3 \mathrm{~V}$; 9-inverter ring oscillator frequency as a function of PMOS and NMOS width 36

Figure 20: 9-inverter ring oscillator layout where each inverter consists of one $0.15 \mu \mathrm{m}$ width PMOS and one $0.12 \mu \mathrm{m}$ width NMOS 38

Figure 21: $65 \mathrm{~nm} \mathrm{LP}, V_{D D}=0.3 \mathrm{~V}$; 9-inverter ring oscillator frequency versus PMOS multiplicity for an NMOS multiplicity of 20 44 
Figure 22: Inverter split into parallel transistors

Figure 23: Double stack configurations for (a) PMOS and (b) NMOS

Figure 24: $65 \mathrm{~nm} \mathrm{LP}, V_{D D}=0.3 \mathrm{~V}$; current-to-capacitance ratio $\left(I_{D} /\left(C_{G}+C_{S B}\right)\right)$ versus width for two series (a) PMOS and (b) NMOS.

Figure 25: $65 \mathrm{~nm} \mathrm{GP}, V_{D D}=0.3 \mathrm{~V}$; current-to-capacitance ratio $\left(I_{D} /\left(C_{G}+C_{S B}\right)\right)$ versus width for two series (a) PMOS and (b) NMOS. 52

Figure 26: $90 \mathrm{~nm}, V_{D D}=0.3 \mathrm{~V}$; current-to-capacitance ratio $\left(I_{D} /\left(C_{G}+C_{S B}\right)\right)$ versus width for two series (a) PMOS and (b) NMOS 53

Figure 27: (a) Standard and (b) parallel inverter configurations for 9-inverter ring oscillator 54

Figure 28: (a) Standard and (b) parallel 3-input NAND gate configurations for 9-gate ring oscillator 54

Figure 29: NAND split into parallel transistors 57

Figure 30: Test circuit schematic 59

Figure 31: Valency-4 carry-lookahead adder block 70

Figure 32: NAND gate from parallel stacks design 72

Figure 33: Standard design carry-lookahead adder layout 73 


\section{TABLE OF SYMBOLS}

\begin{tabular}{|l|l|}
\hline Symbol & Definition \\
\hline$\alpha$ & Activity factor \\
\hline$b$ & Branching effort \\
\hline$\beta$ & Beta ratio, or PMOS-to-NMOS width ratio \\
\hline$\beta_{e f f}$ & Effective beta ratio \\
\hline$\beta_{o p t}$ & Optimum beta ratio \\
\hline$C$ & Capacitance \\
\hline$C_{\text {bottom }}$ & Diffusion bottom-plate capacitance \\
\hline$C_{D B}$ & Drain-to-body diffusion capacitance \\
\hline$C_{d \text { dff }}$ & Diffusion capacitance \\
\hline$C_{e f f}$ & Effective capacitance \\
\hline$C_{G}$ & Gate capacitance \\
\hline$C_{G C}$ & Gate-to-channel capacitance \\
\hline$C_{G D O}$ & Drain overlap capacitance \\
\hline$C_{G S O}$ & Source overlap capacitance \\
\hline$C_{J}$ & Junction capacitance per unit area \\
\hline$C_{I S w}$ & Junction capacitance per unit perimeter \\
\hline$C_{L}$ & Load capacitance \\
\hline$C_{o}$ & Overlap capacitance per unit width \\
\hline$C_{O x}$ & Gate oxide capacitance per unit area \\
\hline$C_{S B}$ & Source-to-body diffusion capacitance \\
\hline$C_{S w}$ & Diffusion side-wall capacitance \\
\hline$E$ & Energy \\
\hline$f$ & (i) Frequency; (ii) stage effort \\
\hline$f_{\max }$ & Maximum frequency \\
\hline$g$ & Logical effort \\
\hline$h$ & Electrical effort, or fanout \\
\hline$G N D$ & Ground \\
\hline$I$ & Current \\
\hline$I_{a v g}$ & Average current \\
\hline$I_{D}$ & Drain current \\
\hline$I_{D S O}$ & Current at threshold \\
\hline$I_{\text {leakage }}$ & Leakage current \\
\hline$i_{\text {peak }}$ & Supply voltage maximum current \\
\hline$I_{S t a t i c}$ & Static current \\
\hline$i_{s u p p l y}$ & Supply voltage instantaneous current \\
\hline$\lambda_{d}$ & Transistor length \\
\hline$L_{S}$ & Drain-induced barrier lowering coefficient \\
\hline & Junction side-wall length \\
\hline & \\
\hline & \\
\hline
\end{tabular}




\begin{tabular}{|l|l|}
\hline$m$ & Empirical parameter \\
\hline$N$ & Number of gates in ring oscillator \\
\hline$n$ & Process-dependent term describing depletion region characteristics \\
\hline$p$ & (i) instantaneous power; (ii) parasitic delay \\
\hline$P_{\text {avg }}$ & Average power \\
\hline$P_{\text {dynamic }}$ & Dynamic power \\
\hline$P_{\text {peak }}$ & Peak power \\
\hline$P_{\text {statc }}$ & Static power \\
\hline$R$ & Resistance \\
\hline$R_{e q}$ & Equivalent resistance \\
\hline$R_{\text {eqn }}$ & NMOS equivalent resistance \\
\hline$R_{\text {eqp }}$ & PMOS equivalent resistance \\
\hline$T$ & (i) Period of oscillation; (ii) time interval \\
\hline$t$ & Time \\
\hline$\tau$ & RC time constant \\
\hline$t_{f}$ & Fall time \\
\hline$t_{p}$ & Propagation delay \\
\hline$t_{p H L}$ & High-to-low propagation delay \\
\hline$t_{p L H}$ & Low-to-high propagation delay \\
\hline$t_{r}$ & Rise time \\
\hline$V_{D D}$ & Supply voltage \\
\hline$V_{D S}$ & Drain-to-source voltage \\
\hline$V_{G S}$ & Gate-to-source voltage \\
\hline$V_{\text {out }}$ & Output voltage \\
\hline$V_{\text {supply }}$ & Supply voltage \\
\hline$V_{\text {swing }}$ & Logic low to logic high voltage range \\
\hline$V_{T}$ & Threshold voltage \\
\hline$v_{T}$ & Thermal voltage \\
\hline$W$ & Transistor width \\
\hline$W_{\text {eff }}$ & Effective width \\
\hline$W_{n}$ & NMOS width \\
\hline$W_{n, o p t}$ & Optimum NMOS width \\
\hline$W_{p}$ & PMOS width \\
\hline$W_{p, o p t}$ & Optimum PMOS width \\
\hline$x_{d}$ & Lateral diffusion \\
\hline & \\
\hline
\end{tabular}


TABLE OF ABBREVIATIONS

\begin{tabular}{|l|l|}
\hline Abbreviations & Definition \\
\hline AC & Alternating current \\
\hline CMOS & Complementary metal-oxide-semiconductor \\
\hline DIBL & Drain-induced barrier lowering \\
\hline EDP & Energy-delay product \\
\hline GP & General purpose transistor variant \\
\hline INV & Logic gate that implements negation \\
\hline INWE & Inverse-narrow-width effect \\
\hline LOCOS & Local oxidation of silicon \\
\hline LP & Low-power transistor variant \\
\hline MOSFET & Metal-oxide-semiconductor field-effect transistor \\
\hline NAND & Logic gate that implements negated AND \\
\hline NAND2 & 2-input logic gate that implements negated AND \\
\hline NAND3 & 3-input logic gate that implements negated AND \\
\hline NMOS & n-channel MOSFET \\
\hline NOR & Logic gate that implements negated OR \\
\hline NOR2 & 2-input logic gate that implements negated OR \\
\hline NOR3 & 3-input logic gate that implements negated OR \\
\hline NWE & Narrow-width effect \\
\hline PDP & Power-delay product \\
\hline PG & Propagate and generate \\
\hline PLS & Post-layout simulations \\
\hline PMOS & p-channel MOSFET \\
\hline PTR & Parallel transistor ratio \\
\hline STI & Shallow trench isolation \\
\hline VLSI & Very-large-scale integration \\
\hline
\end{tabular}




\section{CHAPTER 1 - INTRODUCTION}

Low-power design is an important concept for today's digital circuits. The driving motivator may be different but the goals of lower power dissipation and energy consumption are the same. A desktop computer, for example, is plugged into an AC outlet and so there is no worry about running out of power. However, much effort is placed into the cooling of components to keep them reliable in the heat generated through ever-increasing power dissipation. Laptop computers, smartphones and other portable devices add the need for increased battery life to avoid being tethered to one location for frequent charging. Increasing the battery life can provide a competitive advantage in the marketplace.

In this chapter, the motivations for pursuing a design methodology for subthreshold CMOS logic design are discussed. Also, the objectives of the thesis are presented alongside an outline of how the thesis is organized to meet these objectives.

\subsection{Motivation}

Certain applications, such as battery-operated wireless and medical devices, stress the importance of reduced power dissipation over high speed. Digital subthreshold circuit design has shown promise for low-power applications. In these circuits, the parasitic subthreshold leakage current of superthreshold circuits is used as the primary operation current [1]. It has been demonstrated that the minimum energy operation for CMOS circuits typically occurs in the subthreshold regime [2]. 
While researching the performance of asynchronous circuits $[3,4]$ in subthreshold, an interesting property of the transistor's subthreshold current was noted: the current was not a linear function of the transistor width. In fact, it appeared that the current would decrease with width as expected, but then increase once again at the narrowest widths. It was later observed that the specific current-width relationship would vary between PMOS and NMOS transistors and between technologies. Regardless, it was decided to investigate how to best take advantage of this anomaly.

The primary source of this anomaly is the inverse-narrow-width effect (INWE), the lowering of the threshold voltage as the width of the transistor narrows [5]. This translates into an increase in the subthreshold current due to its exponential dependence on the threshold voltage. Whether a transistor experiences INWE or its opposite, narrowwidth effect (NWE), where the threshold voltage increases with decreasing widths, depends on the method of device isolation (e.g., local oxidation of silicon (LOCOS), shallow trench isolation (STI)) and the p- or n-type dopant implants [6-9]. The effects of different process steps such as additional implants, varied device structures and oxidation methods have also been investigated in regards to the narrow-width effects [10-12].

While analytical expressions for the threshold voltage that account for the narrowwidth effects have been derived, they may prove unwieldy for subthreshold digital design. Also, access to specific process details for a given technology kit may be unavailable. Thus this thesis only demonstrates a design methodology based on the transistor performance that takes advantage of the INWE present in deep submicron technology kits. 


\subsection{Objectives}

The objectives of this thesis are summarized below.

- Understand the transistor subthreshold behavior, in particular with regard to the currents and capacitances.

- Determine how to exploit the subthreshold behavior to maximize performance by reducing the propagation delay of CMOS digital logic circuits.

- Create a design methodology that maximizes subthreshold CMOS logic design performance.

- Demonstrate the effectiveness of the proposed design methodology through the use of application circuits.

\subsection{Organization}

The early chapters detail the relevant theory with which to formulate a design methodology. Chapter 2 focuses on the properties of the transistor such as current and how it is affected by the threshold voltage. A qualitative description of the cause of the narrow-width effects is provided. Leakage currents and the relevant capacitances are also examined. In Chapter 3, important VLSI concepts are discussed. These include propagation delay, power dissipation and energy consumption, as well as other quality metrics such as the power-delay product and the energy-delay product. A description of ring oscillator circuits follows, and the chapter concludes with a brief discussion of the use of Logical Effort as a gate sizing strategy to reduce delay. 
For Chapter 4, the simulation results of actual technology kits are presented and a design methodology is developed. Starting from the transistor properties and their current-to-capacitance ratio, the simulations advance to ring oscillators which validate the optimum widths of transistors to use in subthreshold. The concept of the parallel transistor ratio is introduced. From the subthreshold optimum widths and the parallel transistor ratio, an effective beta ratio, or effective PMOS-to-NMOS width ratio, can be found for use in sizing strategies such as Logical Effort. Simulations of transistor stacks reveal the ineffectiveness of transistor scaling to replicate the current of a single transistor, reinforcing the usage of parallel stacks. Finally, all the steps of the design methodology are summarized.

In Chapter 5, the performance of a circuit designed with the custom methodology is compared to that of a standard design. Results from a minimum-sized circuit are also included. The circuits used for the comparison are a custom test circuit and a 32-bit carry-lookahead adder.

Chapter 6 summarizes the conclusions of this thesis and suggests possible future directions in which to expand upon this work. 


\section{CHAPTER 2 - MOS TRANSISTOR PROPERTIES}

In this chapter, the relevant transistor properties such as current and capacitance are detailed, a qualitative explanation of the inverse-narrow-width effect is given, and sources of leakage are discussed.

\subsection{Currents}

Since operation in the subthreshold regime is the focus of this thesis, the reader is encouraged to read [13] and [15] for details on the transistor superthreshold drain current. While typically undesirable in superthreshold operation, subthreshold current is the name given to the drain current that occurs in subthreshold, and is discussed in the next section. The junction leakage and gate leakage currents are also discussed.

\subsubsection{Subthreshold Current}

When the gate-to-source voltage $\left(V_{G S}\right)$ is less than the threshold voltage $\left(V_{T}\right)$, current between the drain and source of a MOSFET is still possible due to weakinversion conduction. An expression for this subthreshold current is given by

$$
I_{D}=I_{D S 0} e^{\frac{V_{G S}-V_{T}}{n \nu_{T}}}\left(1-e^{\frac{-V_{D S}}{v_{T}}}\right)
$$

where $I_{D S O}$ is the current at the threshold that depends on process and device geometry, $n$ is a process-dependent term describing the depletion region characteristics with a value typically in the range of 1.4 to $1.5, v_{T}$ is the thermal voltage and $V_{D S}$ is the drain-tosource voltage [13]. 
Equation (1) shows the exponential dependence of the subthreshold current on the threshold voltage. Contrast this to the superthreshold current where the dependence on the threshold voltage is between linear and square depending on the severity of the carrier velocity saturation [14]. In superthreshold operation, the subthreshold current is called leakage and is undesirable. Here, it is the primary operation current $[1,13]$.

\subsubsection{Junction Leakage}

The p-n junctions between the source and drain diffusions and the substrate or well form diodes. Another diode is formed between a well and the substrate. The wells and substrate are biased to $V_{D D}$ or $G N D$ to ensure a reverse-bias. Yet reverse-biased diodes still conduct a small amount of current referred to as junction leakage [13]. The low-power transistor variant in modern technology kits reduces this leakage.

\subsubsection{Gate Leakage}

Through a process called tunneling, charge carriers can pass through the gate oxide. Thinner oxides exponentially increase the opportunity for tunneling to occur [13]. As with the junction leakage, the low-power transistor variants reduce the gate leakage current.

\subsubsection{Current Comparison}

Tables 1 and 2 compare the drain, junction leakage and gate leakage currents that occur within a typical $65 \mathrm{~nm}$ process with general purpose (GP) and low-power (LP) 
transistor variants at $1 \mathrm{~V}$ and $0.3 \mathrm{~V}$. At both voltages, the junction and gate leakage currents are several orders of magnitude below the drain current thus supporting the use of the subthreshold drain current as the primary operation current. Also worth noting is that in superthreshold, the GP transistors conduct approximately twice the current of the LP variants, whereas in subthreshold, they conduct at least 30 times the current. This is an indication of how the low-power variant is effective at reducing the subthreshold current which in typical superthreshold applications is undesired.

Table 1: $65 \mathrm{~nm}, V_{D D}=1 \mathrm{~V}$; currents for $0.12 \mu \mathrm{m}$ width transistors

\begin{tabular}{|l|c|c|c|c|}
\hline \multicolumn{1}{|c|}{ Current } & $\begin{array}{c}\text { PMOS GP } \\
{[\boldsymbol{\mu A}]}\end{array}$ & $\begin{array}{c}\text { NMOS GP } \\
{[\boldsymbol{\mu A}]}\end{array}$ & $\begin{array}{c}\text { PMOS LP } \\
{[\boldsymbol{\mu A}]}\end{array}$ & $\begin{array}{c}\text { NMOS LP } \\
{[\boldsymbol{\mu A}]}\end{array}$ \\
\hline Drain & $6.0 \times 10^{1}$ & $1.1 \times 10^{2}$ & $3.1 \times 10^{1}$ & $5.3 \times 10^{1}$ \\
\hline Junction leakage & $4.0 \times 10^{-7}$ & $3.8 \times 10^{-5}$ & $6.0 \times 10^{-9}$ & $1.2 \times 10^{-5}$ \\
\hline Gate leakage & $3.7 \times 10^{-4}$ & $4.6 \times 10^{-4}$ & $7.6 \times 10^{-8}$ & $2.7 \times 10^{-7}$ \\
\hline
\end{tabular}

Table 2: $65 \mathrm{~nm}, V_{D D}=0.3 \mathrm{~V}$; currents for $0.12 \mu \mathrm{m}$ width transistors

\begin{tabular}{|l|c|c|c|c|}
\hline \multicolumn{1}{|c|}{ Current } & $\begin{array}{c}\text { PMOS GP } \\
{[\boldsymbol{\mu A}]}\end{array}$ & $\begin{array}{c}\text { NMOS GP } \\
{[\boldsymbol{\mu} \mathbf{A}]}\end{array}$ & $\begin{array}{c}\text { PMOS LP } \\
{[\boldsymbol{\mu A}]}\end{array}$ & $\begin{array}{c}\text { NMOS LP } \\
{[\boldsymbol{\mu} \mathbf{A}]}\end{array}$ \\
\hline Drain & 2.0 & 1.2 & $1.9 \times 10^{-2}$ & $3.6 \times 10^{-2}$ \\
\hline Junction leakage & $2.3 \times 10^{-9}$ & $1.2 \times 10^{-7}$ & $7.3 \times 10^{-11}$ & $1.9 \times 10^{-11}$ \\
\hline Gate leakage & $1.8 \times 10^{-5}$ & $8.2 \times 10^{-6}$ & $3.9 \times 10^{-9}$ & $2.4 \times 10^{-9}$ \\
\hline
\end{tabular}

\subsection{Capacitances}

The gate and diffusion capacitances, discussed in the next two sections, are the capacitances relevant to digital logic design. 


\subsubsection{Gate Capacitance}

The gate capacitance $\left(C_{G}\right)$ consists of three components: the gate-to-channel capacitance $\left(C_{G C}\right)$ and the two overlap capacitances $\left(C_{G S O}\right.$ for the source and $C_{G D O}$ for the drain). The gate-to-channel capacitance can be divided into three components that change in magnitude with the terminal voltages or operation region. These are the gateto-source, gate-to-drain and gate-to-body capacitances. In superthreshold, the values of these component capacitances are often estimated for the operation region to ease analysis. If considering the gate capacitance as a load, these components may not matter since only the lumped total value will matter. The value of the gate capacitance does not vary much between the operation regions. If operating in subthreshold, this variation is further reduced.

The gate-to-channel capacitance can be approximated as

$$
C_{G C}=C_{o x} W L
$$

where $C_{o x}$ is the gate oxide capacitance per unit area, $W$ is the width and $L$ is the length of the channel. The other components of the gate capacitance, the overlap capacitances, are only a function of the structure of the transistor. They are caused by the extension of the source and drain diffusions under the gate. The polysilicon gate and the source or drain form the plates of the capacitor while the thin oxide is the dielectric. They are expressed as

$$
C_{G S o}=C_{G D o}=C_{o x} x_{d} W=C_{o} W
$$


where $x_{d}$ is the length of the extension of the source or drain under the gate oxide. Since $x_{d}$ is process dependent, the overlap capacitance is often expressed in terms of the overlap capacitance per unit width $C_{o}$. Summing Equations (2) and (3), the gate capacitance can be estimated as [15]

$$
C_{G}=C_{G C}+C_{G S O}+C_{G D O}=C_{o x} W L+2 C_{o} W
$$

From Equation (4), it can be seen that the gate capacitance scales with transistor width.

\subsubsection{Diffusion Capacitance}

The diffusions for the source and drain withın the substrate form reverse-biased p-n junctions which contribute the $C_{S B}$ (source-to-body) and the $C_{D B}$ (drain-to-body) parasitic capacitances, respectively. The depletion region width increases with the reverse-bias voltage, thus the capacitance is maximized when the source or drain and body are at the same voltage. Since the diffusion sits as a tub within the substrate, its capacitance is expressed as the sum of two junction capacitances: bottom-plate area and side-wall perimeter. The bottom-plate capacitance is given as

$$
C_{\text {bottom }}=C_{J} W L_{S}
$$

where $C_{J}$ is the junction capacitance per unit area, $W$ is the diffusion width (same as transistor width) and $L_{S}$ is the side-wall length. The side-wall capacitance is

$$
C_{s w}=C_{j s w}\left(W+2 L_{S}\right)
$$

where $C_{J s w}$ is the capacitance per unit perimeter. Note that for a first-order analysis, the diffusion-to-channel capacitance is ignored. 
The total diffusion capacitance is then [15]

$$
C_{d \imath f f}=C_{b o t t o m}+C_{s w}=C_{J} W L_{S}+C_{J s w}\left(W+2 L_{S}\right)
$$

When transistors are in series, it is common to share the diffusion areas. The drain of one transistor is merged with the source of another. Depending on whether a contact is required, the side-wall length may vary [13]. Similar to the gate capacitance, the diffusion capacitance scales almost linearly with the width.

\subsection{Narrow-Width Effects}

The inverse-narrow-width effect (INWE) is the reduction of the threshold voltage as the width of a transistor narrows. Conversely, the narrow-width effect (NWE) increases the threshold voltage as the transistor width narrows. As seen in Section 2.1.1, this could have a large effect on the subthreshold current because of its exponential dependence on the threshold voltage. Only a qualitative description of the narrow-width effects will be provided here since the subthreshold design methodology of this thesis does not depend on the exact mechanism or modeling of the INWE.

The predominant cause of the narrow-width effects is the type of device isolation used. Due to the limitations of the fabrication process, the polysilicon gate of a MOSFET extends beyond the channel width on both sides. This excess polysilicon overlaps the field oxide, creating a fringing capacitance. In the case of a transistor where shallow trench isolation (STI) is used, the fringing electric field lines terminate on charge located within the device channel, as shown in Figure 1(a). Thus the total gate-to-channel 
capacitance consists of the typical parallel plate thin oxide capacitance plus the fringing capacitance. As the transistor width narrows, the fringing capacitance becomes more dominant in proportion to the parallel plate capacitance. Consequently, at narrower widths, the creation of an inversion layer is facilitated at a lower applied gate voltage, resulting in the INWE.

The fringing electric field lines of a transistor where local oxidation of silicon (LOCOS) is used terminate on charge located in the substrate, as seen in Figure 1(b). Also, the "bird's beak" encroachment of the silicon dioxide $\left(\mathrm{SiO}_{2}\right)$ effectively reduces the designed width of the transistor, leading to a reduction in the gate-to-channel capacitance. At narrower widths, the proportion of charge accumulated in the channel is reduced compared to the charge accumulated in the substrate, thus necessitating a higher gate voltage to produce an inversion layer. This is termed the NWE $[5,8]$.

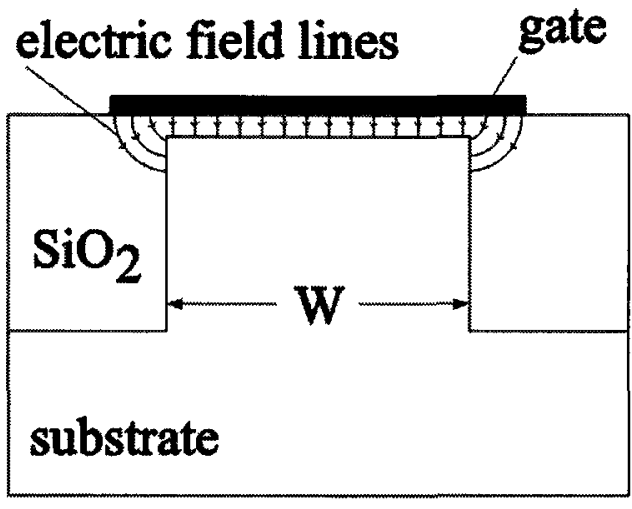

(a)

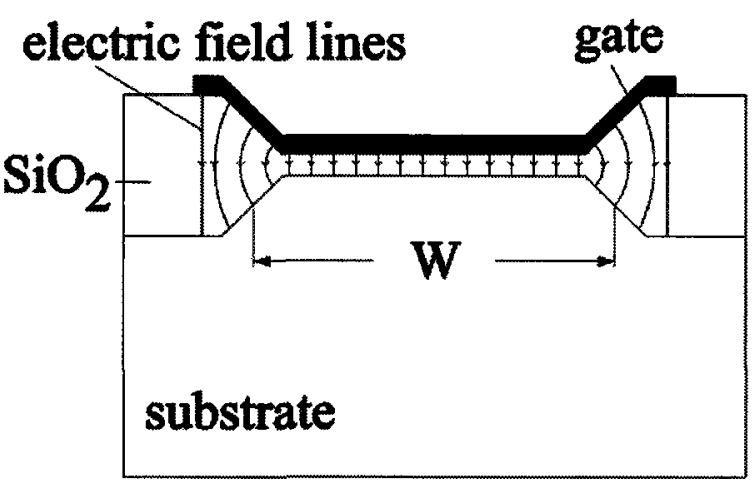

(b)

Figure 1: Cross section of a MOSFET fabricated with (a) shallow trench isolation and (b) local oxidation of silicon 


\section{CHAPTER 3 - VLSI CONCEPTS}

Metrics and methods for characterizing and evaluating the performance of CMOS circuits such as propagation delay and power dissipation are discussed in this chapter. The relevant properties of ring oscillator circuits are detailed and Logical Effort, a delay estimation and circuit optimization technique, is also reviewed.

\subsection{Propagation Delay}

The propagation delay $t_{p}$ defines how quickly a logic gate responds to a change at its input(s). It is measured as the time interval between the $50 \%$ transition points of the input and output waveforms. Since a gate's response varies for rising and falling signals, two definitions of the propagation delay are required. $t_{p H L}$ represents the propagation delay when the output transitions from high-to-low, while $t_{p L H}$ covers the low-to-high transition. The propagation delay is then the average of the two:

$$
t_{p}=\frac{t_{p L H}+t_{p H L}}{2}
$$

In order to estimate the propagation delay, digital circuits are typically modeled as first-order $R C$ networks. The transistor current is modeled by a constant linear resistance whose value is averaged over the interval of interest. The load capacitance is also modeled as a constant factor over this interval. While only diffusion and gate capacitances are typically considered for hand calculations, in reality, interconnect capacitances will also affect the performance. 
If we assume a step input going from 0 to $V$ is applied to a first-order $R C$ network, the transient response is given by

$$
v_{\text {out }}(t)=\left(1-e^{-t / \tau}\right) V
$$

where $\tau=R C$, the time constant of the network.

From Equation (9), the time for the output to reach $50 \%$ of its final value can be determined as

$$
t=\ln (2) R C=0.69 R C
$$

For digital circuits, a first-order estimate of the propagation delay is simply a matter of replacing $R$ with the equivalent resistance $R_{e q}$ of the transistor(s) and $C$ with the load capacitance $C_{L}$ in Equation (10). In most cases, the PMOS pull-up network and the NMOS pull-down network of a gate will have different equivalent resistances, represented by $R_{e q p}$ and $R_{e q n}$, used to calculate $t_{p L H}$ and $t_{p H L}$, respectively. Equation (8) can then be used to estimate the propagation delay.

Another equation for the propagation delay derived from the definition of current is

$$
t_{p}=\frac{c_{L}\left(V_{\text {swing }} / 2\right)}{I_{\text {avg }}}
$$

where $V_{\text {swing }}$ is the voltage range from logic low to logic high and $I_{\text {avg }}$ is the average current over the period of interest [15]. 


\subsection{Rise and Fall Times}

Rise and fall times are properties of individual signals rather than gates. In the previous section, the estimate for propagation delay assumed a step input signal. The actual delay will be longer for inputs with lower slopes. Rise and fall times are used to quantify the transition rate of a signal. To avoid any uncertainty over where a transition begins and ends, the rise and fall times use the $10 \%$ and $90 \%$ points of a waveform. Thus the rise time, $t_{r}$, is the time for a signal to reach the $90 \%$ point from the $10 \%$ point in a rising transition, while the fall time, $t_{f}$, is the time for a $90 \%$ to $10 \%$ signal transition in a falling transition [15].

\subsection{Power Dissipation}

The peak power dissipation is important to consider when sizing supply lines. If the instantaneous power is $p(t)$, the peak power, $P_{\text {peak }}$, is defined as

$$
P_{\text {peak }}=i_{\text {peak }} V_{\text {supply }}=\max [p(t)]
$$

where $V_{\text {supply }}$ is the supply voltage and $i_{\text {peak }}$ is the maximum current drawn from it.

More important is the average power, $P_{a v g}$, since this relates directly to battery and cooling requirements. It is defined as

$$
P_{\text {avg }}=\frac{1}{T} \int_{0}^{T} p(t) d t=\frac{V_{\text {supply }}}{T} \int_{0}^{T} i_{\text {supply }}(t) d t
$$

where $i_{\text {supply }}$ is the instantaneous current from the supply voltage and $T$ is the interval of interest. 
Power dissipation can be further broken down into dynamic and static components. The dynamic power dissipation occurs during transients, i.e., when gates are switching. It is caused by the charging of capacitors and temporary current paths between the supply rails. The dynamic power is proportional to the number of switching events. The dynamic power dissipation is

$$
P_{\text {dynamic }}=\alpha \times f \times C_{e f f} \times V_{D D}^{2}
$$

where $\alpha$ is the activity factor, $f$ is the switching frequency, $C_{\text {eff }}$ is the effective capacitance being charged and discharged and $V_{D D}$ is the supply voltage. The static power dissipation occurs at all times, even when the circuit is in steady-state. It is caused by the permanent conductive paths between the supply rails and by leakage currents such as gate leakage and junction leakage, and is described by

$$
P_{\text {statıc }}=I_{\text {leakage }} \times V_{D D}
$$

Operation in subthreshold will clearly have a significant impact in reducing both dynamıc and static power dissipation by lowering the supply voltage seen in Equations (14) and (15). Moreover, many of the leakage currents have a very strong dependence on the terminal voltages. For example, gate leakage current, with its strong dependence on the gate voltage, becomes nearly negligible in subthreshold $[1,15]$.

\subsection{Energy Consumption}

For a first-order $R C$ network with a step input going from 0 to $V$, the total energy $E$ delivered is [15] 


$$
E=C V^{2}
$$

For digital circuits, $C$ will be the load capacitance $C_{L}$ and $V$ is the supply voltage $V_{D D}$. From Equation (14), more energy will be consumed the greater the number of switching events.

\subsection{Power-Delay Product}

The power-delay product (PDP) is a quality measure for a switching device. The unit for power dissipation is the watt, equivalent to joules per second, and time is the measurement for propagation delay. Thus their product will be expressed in joules, the unit of energy. The PDP is therefore defined as the energy consumed by the gate per switching event [15].

\subsection{Energy-Delay Product}

Ideally, logic gates should be fast and consume little energy. The energy-delay product (EDP) can be considered an important quality metric since it combines both of these elements. The EDP can be found by multiplying the PDP by the propagation delay, and will thus have units of joule-seconds [15]. When discussing the efficiency of different circuit implementations, EDP is a reasonable parameter to consider.

\subsection{Ring Oscillator Circuits}

Ring oscillators typically consist of an odd number of inverter gates in a closed loop, where the output of one gate is fed directly as the input of the next. The circuit will 
continue to oscillate due to the odd number of inversions. The period of oscillation can be found as

$$
T=2 \times t_{p} \times N
$$

where $\mathrm{N}$ is the number of inverters. Note the factor of two arises since a complete cycle requires both high-to-low and low-to-high transitions [15].

Ring oscillators can also be built from other gate types. For example, if using NAND gates, both inputs can be tied together or one of the inputs can be tied to $V_{D D}$, depending upon the purpose of the circuit.

\subsection{Logical Effort}

Logical Effort is a method for estimating the delay of a logic circuit, and more importantly, optimizing it. This refers to the sizing of the gates as well as potentially adding buffers to minimize the delay. Logical Effort makes use of two factors that are normalized to an inverter: the parasitic delay, $p$, and the logical effort, $g$. The parasitic delay describes the delay inherent to the gate itself with no load attached. Logical effort, when referring to the factor $g$, describes the increase in input capacitance of a gate to produce the same current as an inverter, or equivalently, the reduction in output current for a gate having the same input capacitance as an inverter. Three other factors are the electrical effort, or fanout, $h$, the stage effort, $f$, and the branching effort, $b$. While these factors apply to individual gates, the same factors are defined separately for a path, or a series of gates. 
A typical application of Logical Effort is sizing the gates of a logic path with a known load and a fixed-sized driving gate. By using equations that relate the path factors to the gate factors, the sizes for the gates that reduce the delay can be found. Also, the optimum number of stages can be determined, thus prompting the addition of buffers if desired $[13,15]$. 


\section{CHAPTER 4 - DESIGN METHODOLOGY DEVELOPMENT}

The subthreshold performance of transistors is process dependent and is unique to each technology kit to some degree. Variations in performance between PMOS and NMOS transistors are also prevalent. In this chapter, data from actual technology kits will be presented. A subthreshold design methodology that takes advantage of the particulars of a given technology kit will be developed.

The main technology kit in use, in particular for the applications of Chapter 5 , is a $65 \mathrm{~nm} 1.2 \mathrm{~V}$ low-power (LP) process. Data from other kits such as a $65 \mathrm{~nm} 1 \mathrm{~V}$ general purpose (GP) process and a $90 \mathrm{~nm} 1.2 \mathrm{~V}$ technology will also be presented to demonstrate that while the specifics may change between kits, the method being developed can still be used to maximize the subthreshold performance of a given technology. Comparisons to superthreshold operation will also feature extensively to highlight the differences in performance at the two supply voltage ranges. It should be noted that the minimum transistor width for these three kits is $0.12 \mu \mathrm{m}$.

\subsection{Subthreshold Optimum Width from Current-to-Capacitance Ratio}

The transistors are configured such that the gate and drain are tied together and connected to the appropriate power supply for maximum current drive as shown in Figure 2. The current, threshold voltage and gate and source diffusion capacitances versus width of both the PMOS and NMOS $65 \mathrm{~nm}$ LP transistors at $1.2 \mathrm{~V}$ are plotted in Figure 3 . The currents and capacitances appear to be nearly linear with width. The threshold voltage drops as the width narrows, consistent with the inverse-narrow-width effect. 


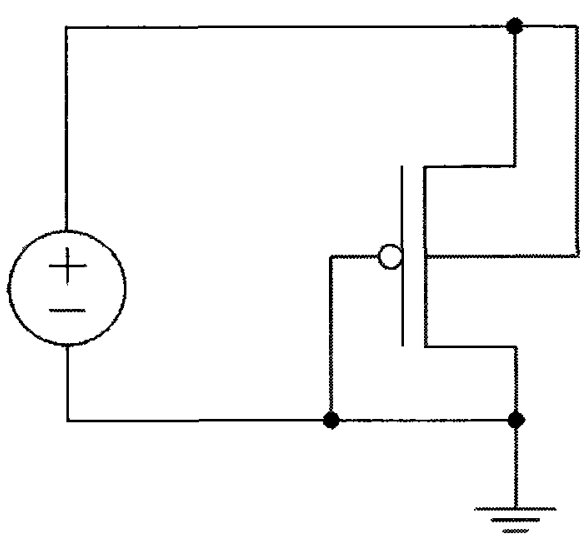

(a)

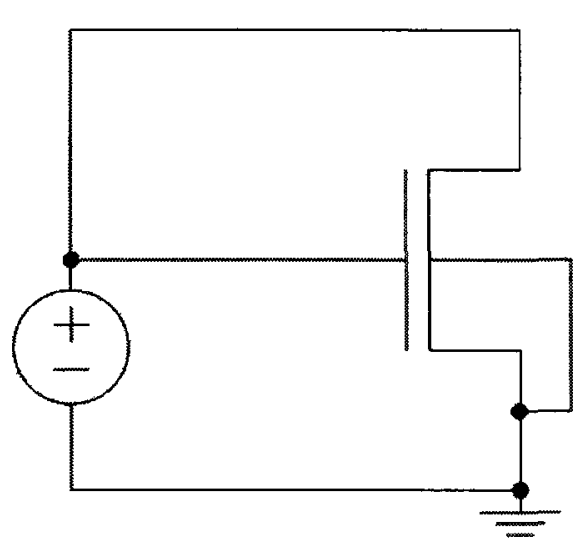

(b)

Figure 2: Transistor configuration for maximum current drive for (a) PMOS and (b) NMOS
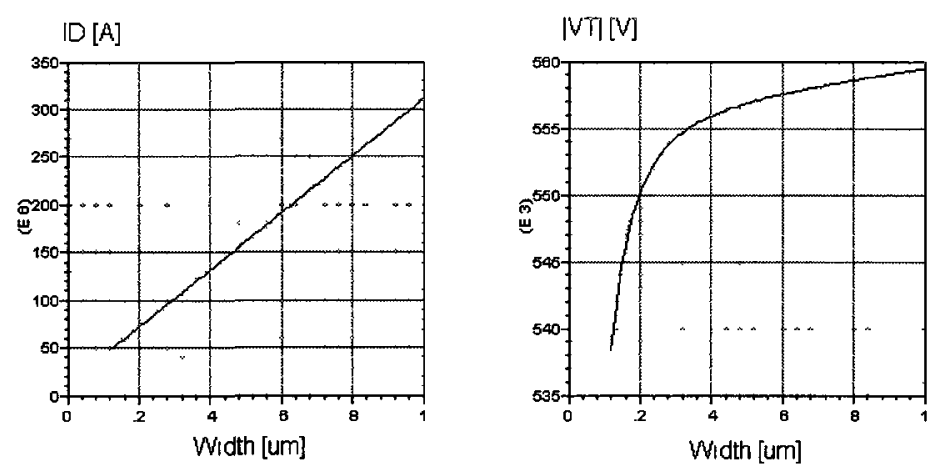

(a)

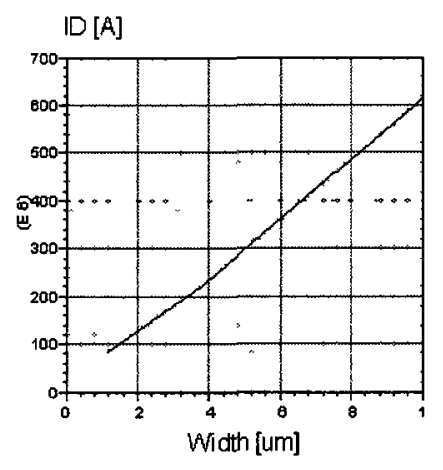

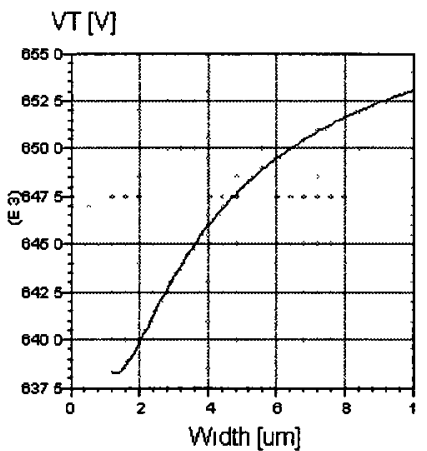

(b)
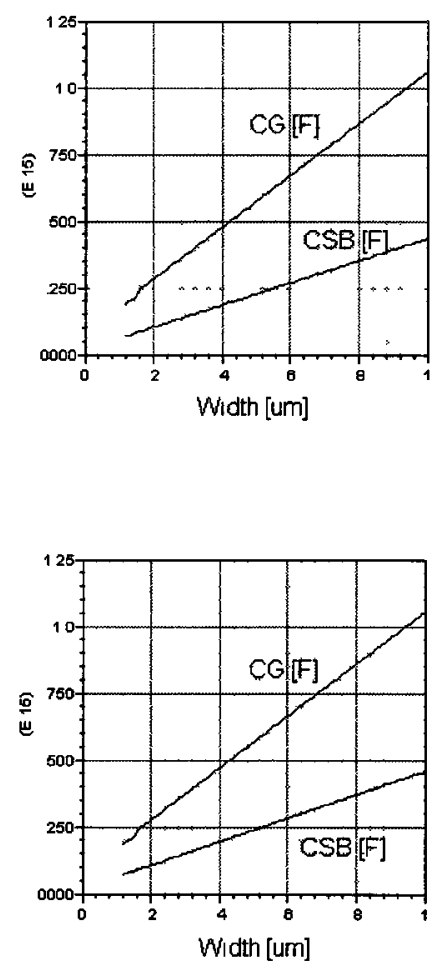

Figure 3: $65 \mathrm{~nm} \mathrm{LP}, V_{D D}=1.2 \mathrm{~V}$; current $\left(I_{D}\right)$, threshold voltage $\left(V_{T}\right)$ and gate $\left(C_{G}\right)$ and source $\left(C_{S B}\right)$ diffusion capacitances versus width for (a) PMOS and (b) NMOS 
The same plots but for a power supply voltage of $0.3 \mathrm{~V}$ are shown in Figure 4 . While the actual values of the threshold voltages and the capacitances vary, they follow the same trend with the width as compared to the superthreshold voltage. The big change is in the current. As expected, the current has dropped significantly, here by over three orders of magnitude. The real surprise is the way in which the current appears to be higher than what would be expected at the narrow widths. While this can be seen in the NMOS transistor, the extreme case is the PMOS transistor where the lowest current does not occur at the narrowest width.
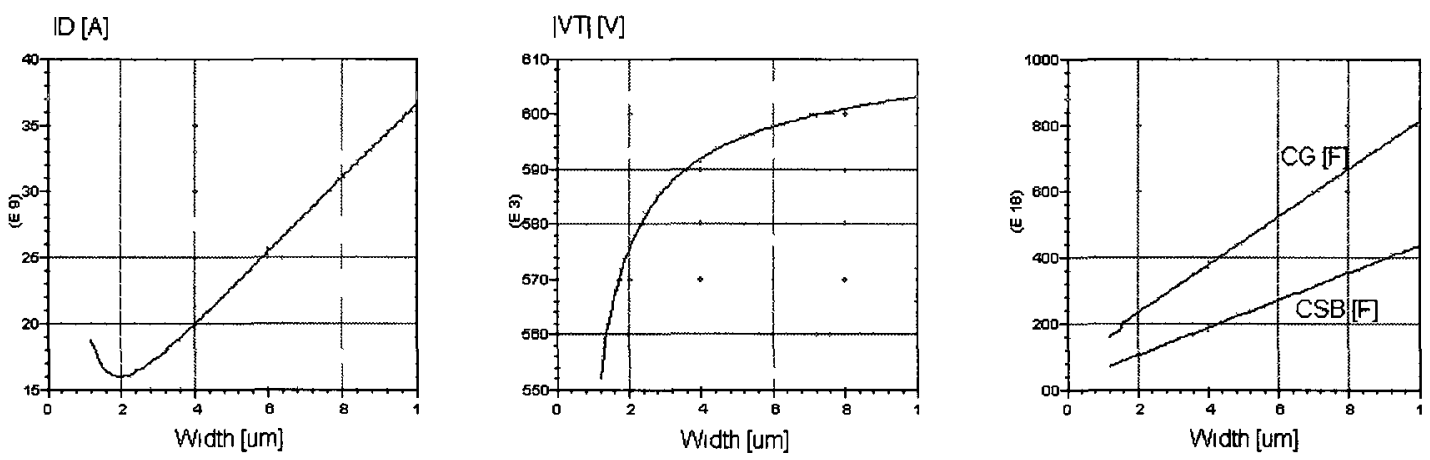

(a)
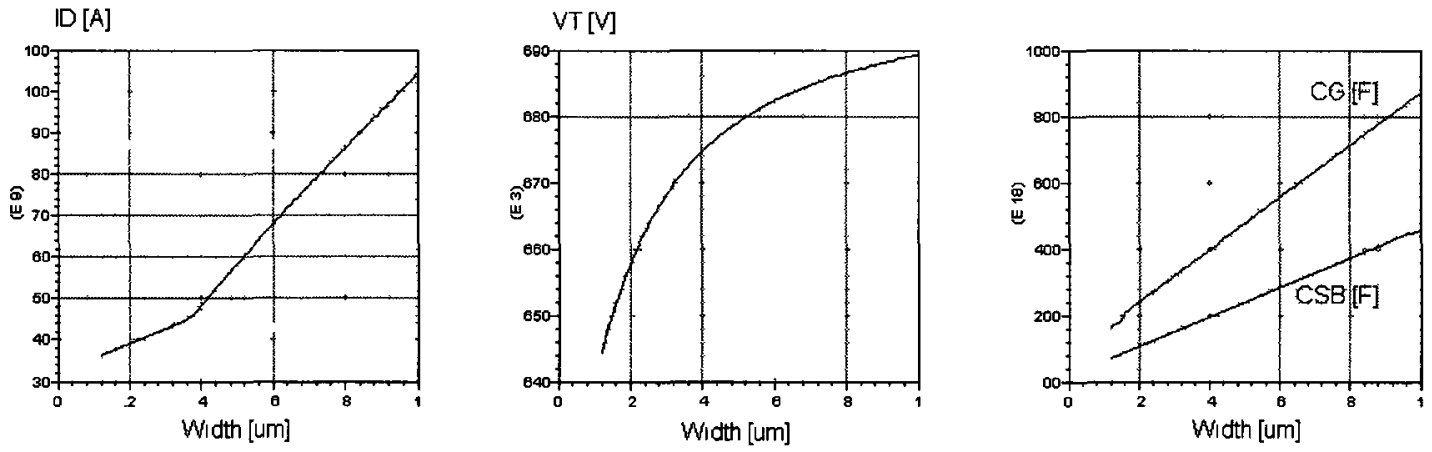

(b)

Figure 4: $65 \mathrm{~nm} \mathrm{LP}, V_{D D}=0.3 \mathrm{~V}$; current $\left(I_{D}\right)$, threshold voltage $\left(V_{T}\right)$ and gate $\left(C_{G}\right)$ and source $\left(C_{S B}\right)$ diffusion capacitances versus width for (a) PMOS and (b) NMOS 
The superthreshold and subthreshold plots for the $65 \mathrm{~nm}$ GP technology are shown in Figures 5 and 6. The subthreshold currents are only between one and two orders of magnitude smaller than the superthreshold case. Again, it is the PMOS transistor that displays the surprising current plot. In fact, at the narrowest widths, the NMOS current is weaker than that of the PMOS.
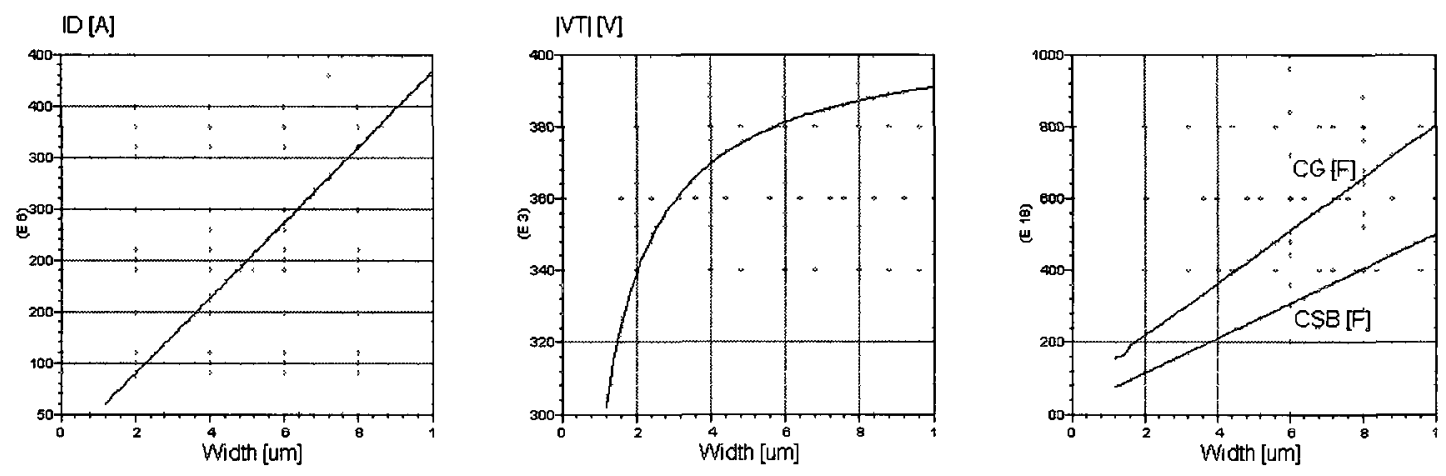

(a)
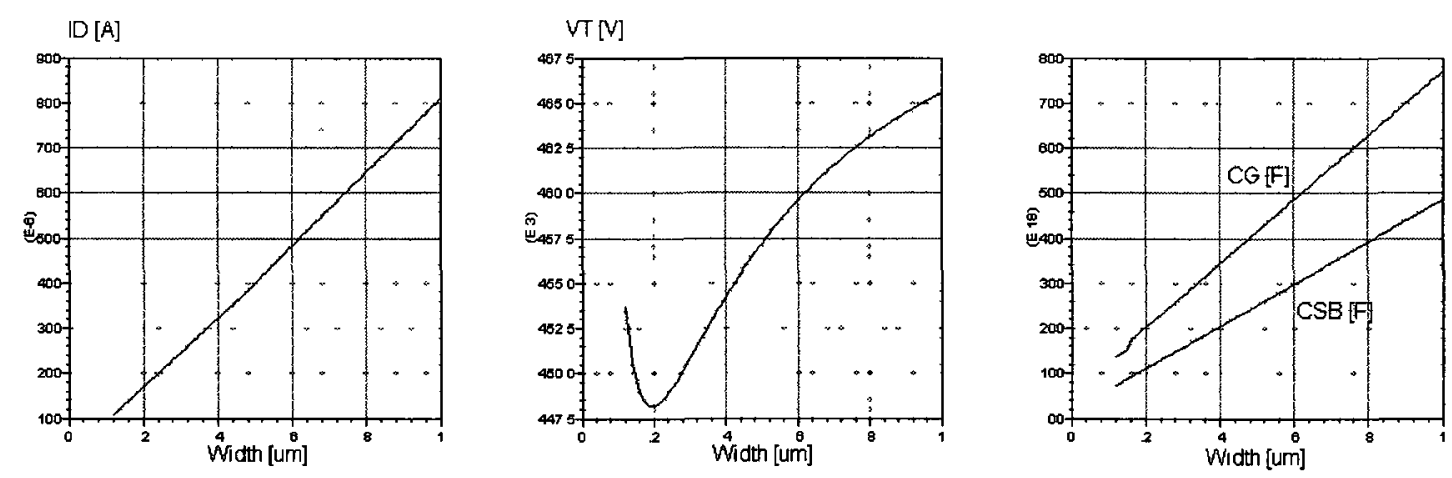

(b)

Figure 5: $65 \mathrm{~nm} \mathrm{GP,} V_{D D}=1.0 \mathrm{~V}$; current $\left(I_{D}\right)$, threshold voltage $\left(V_{T}\right)$ and gate $\left(C_{G}\right)$ and source $\left(C_{S B}\right)$ diffusion capacitances versus width for (a) PMOS and (b) NMOS 

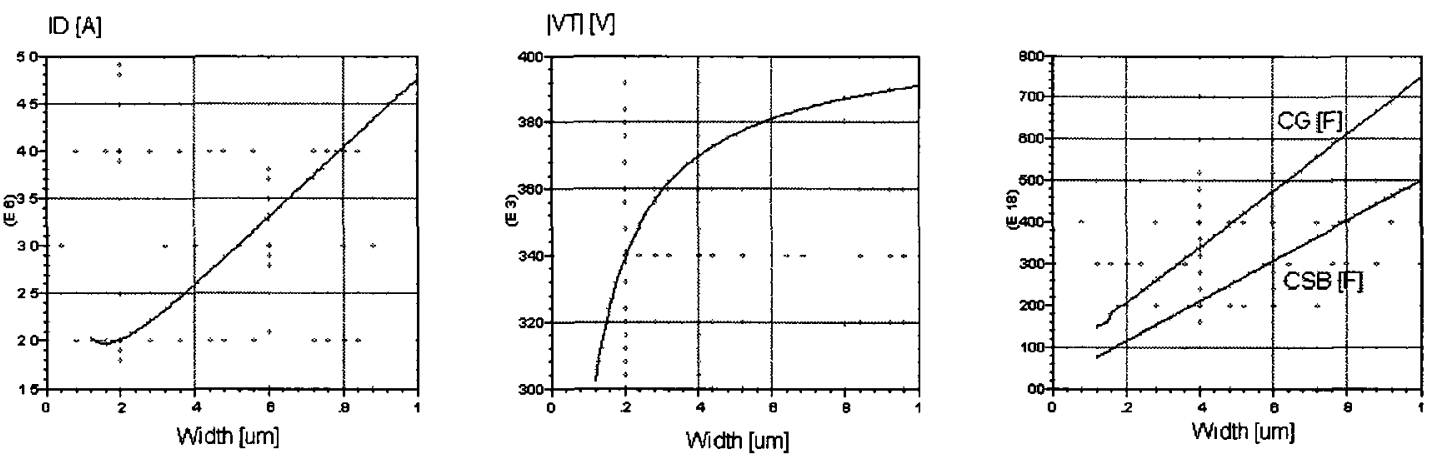

(a)
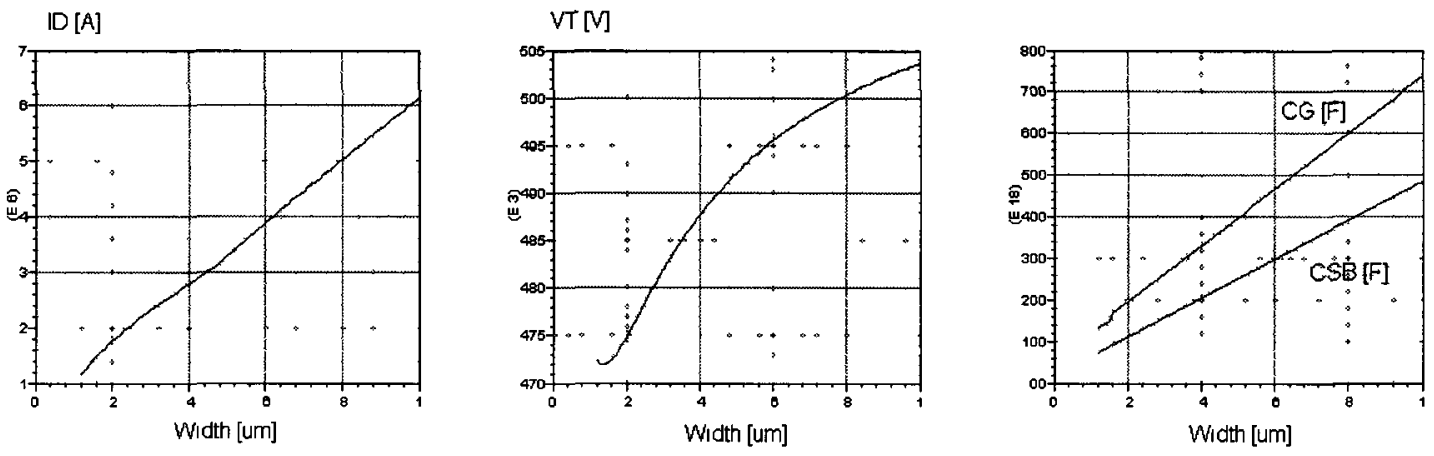

(b)

Figure 6: $65 \mathrm{~nm} \mathrm{GP,} V_{D D}=0.3 \mathrm{~V}$; current $\left(I_{D}\right)$, threshold voltage $\left(V_{T}\right)$ and gate $\left(C_{G}\right)$ and source $\left(C_{S B}\right)$ diffusion capacitances versus width for (a) PMOS and (b) NMOS 
Figures 7 and 8 are plots for the $90 \mathrm{~nm}$ technology kit. The big change here is the PMOS transistor exhibiting narrow-width effect where the threshold voltage increases at the narrowest widths. It is the NMOS transistor that displays the upturn in current at the narrowest widths in subthreshold. Finally, the PMOS subthreshold current is much weaker compared to that of the NMOS.
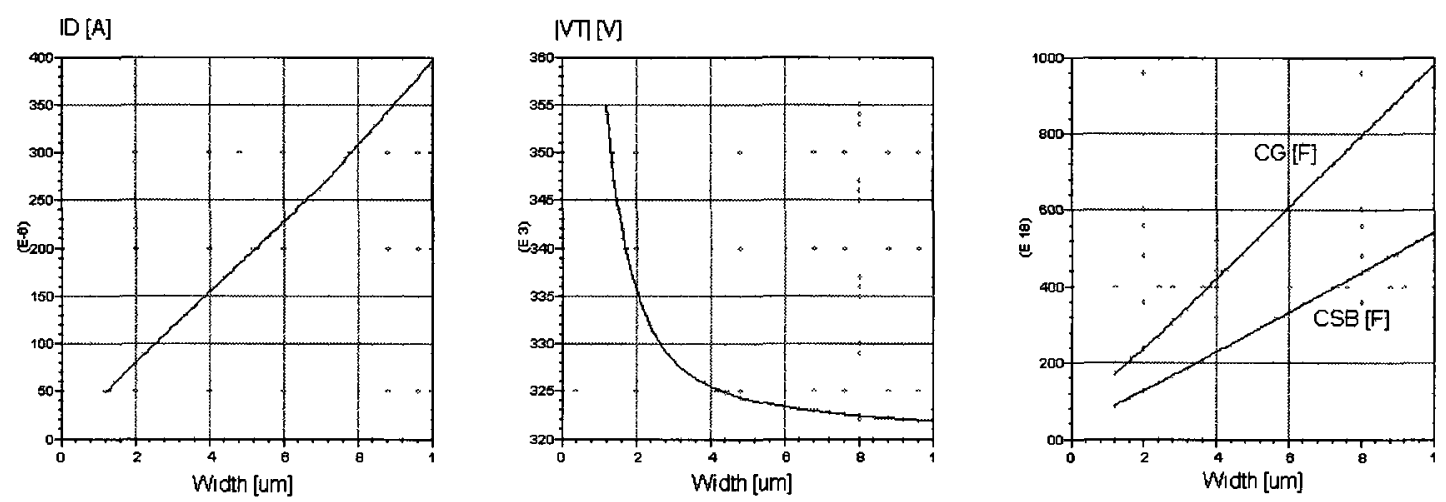

(a)
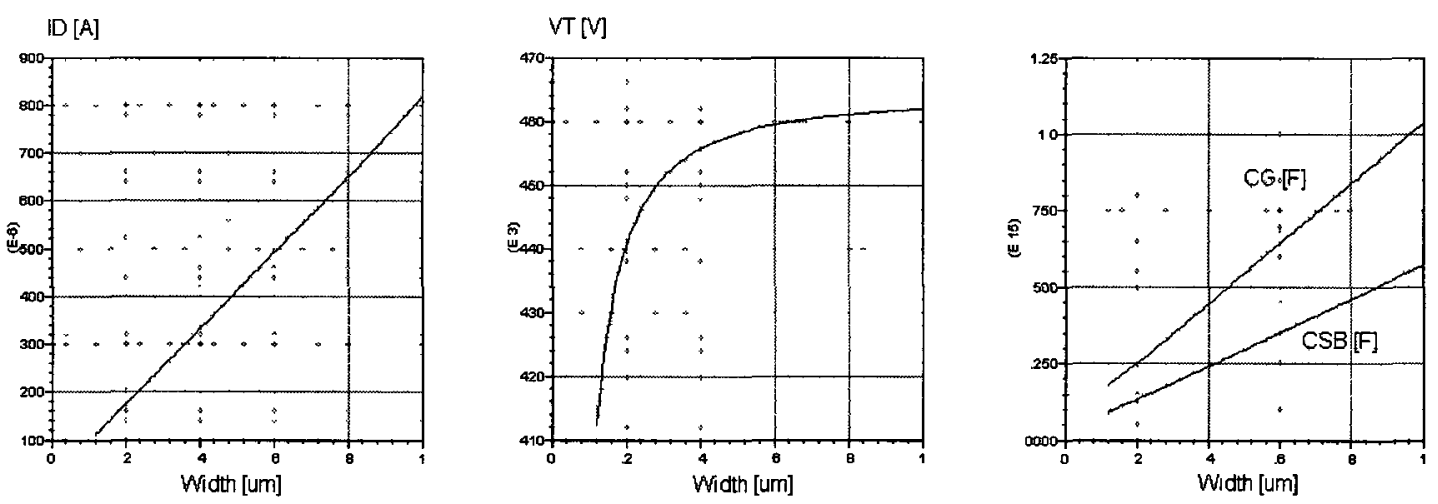

(b)

Figure 7: $90 \mathrm{~nm}, V_{D D}=1.2 \mathrm{~V}$; current $\left(I_{D}\right)$, threshold voltage $\left(V_{T}\right)$ and gate $\left(C_{G}\right)$ and source $\left(C_{S B}\right)$ diffusion capacitances versus width for (a) PMOS and (b) NMOS 

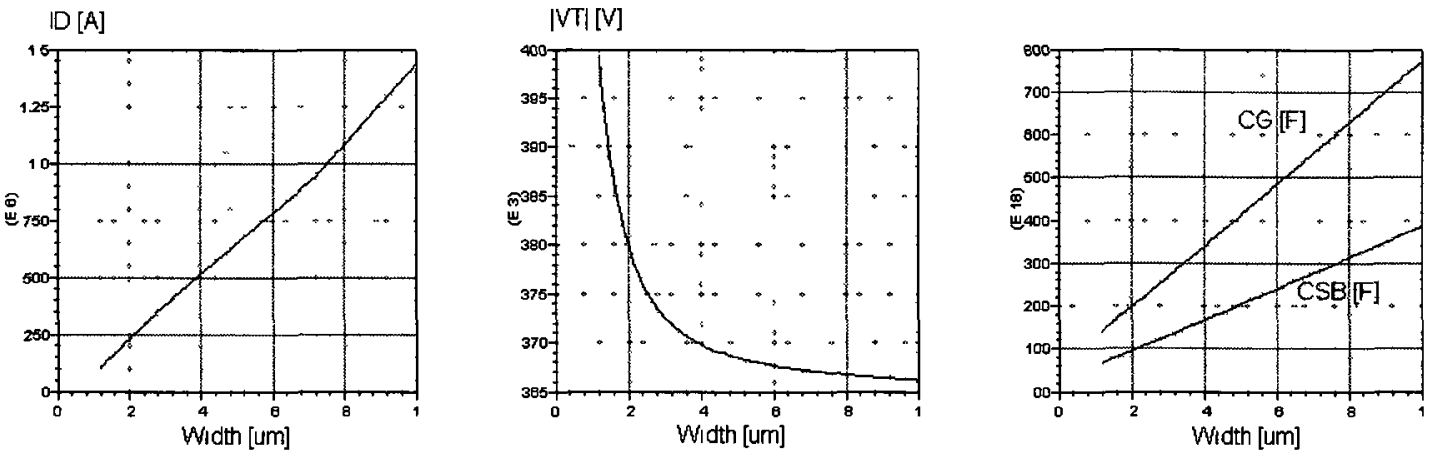

(a)
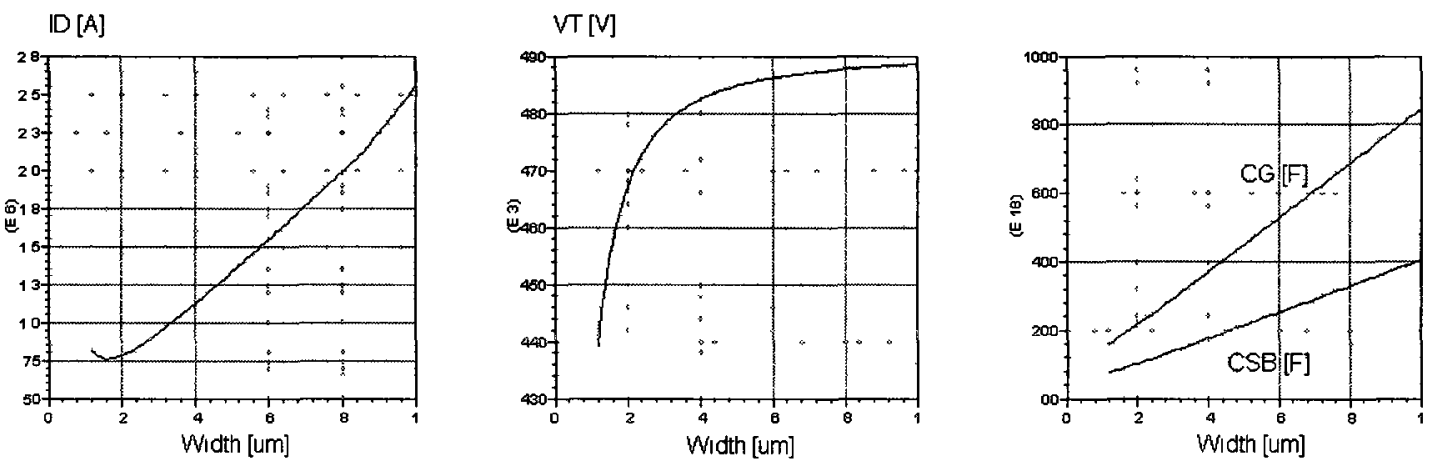

(b)

Figure 8: $90 \mathrm{~nm}, V_{D D}=0.3 \mathrm{~V}$; current $\left(I_{D}\right)$, threshold voltage $\left(V_{T}\right)$ and gate $\left(C_{G}\right)$ and source $\left(C_{S B}\right)$ diffusion capacitances versus width for (a) PMOS and (b) NMOS

As seen in Equation (11), a reduction in propagation delay can be achieved by either increasing the current of the relevant transistors or by reducing the load capacitance. Consistent with what is shown in [16], both goals can be achieved by using transistor widths that maximize the current-to-capacitance ratio. In Figure 9, the currentto-capacitance ratio has been plotted for both the PMOS and NMOS $65 \mathrm{~nm}$ LP transistors in superthreshold. For the capacitance, the sum of the gate and source diffusion capacitances has been used. The gate capacitance is representative of a typical load and 
the diffusion capacitance is representative of the self-loading that occurs in logic gates. The source diffusion capacitance has been used since it represents the worst case diffusion capacitance. As seen in the plot, the ratio continues to increase as the width approaches $1 \mu \mathrm{m}$.

$\mathrm{ID} /(\mathrm{CG}+\mathrm{CSB})[\mathrm{A} / \mathrm{F}]$

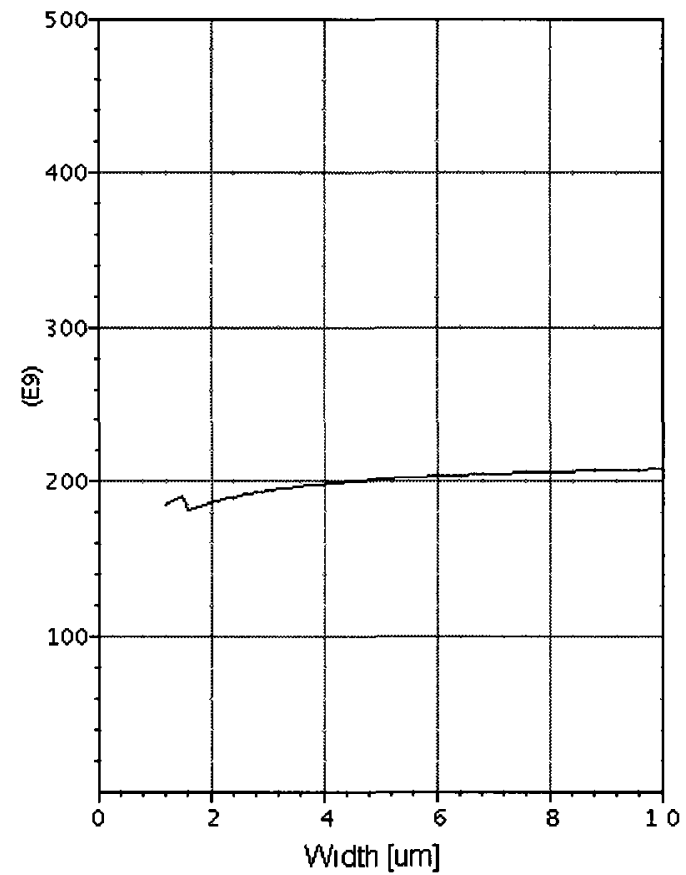

(a)
$\mathrm{ID} /(\mathrm{CG}+\mathrm{CSB})[\mathrm{A} / \mathrm{F}]$

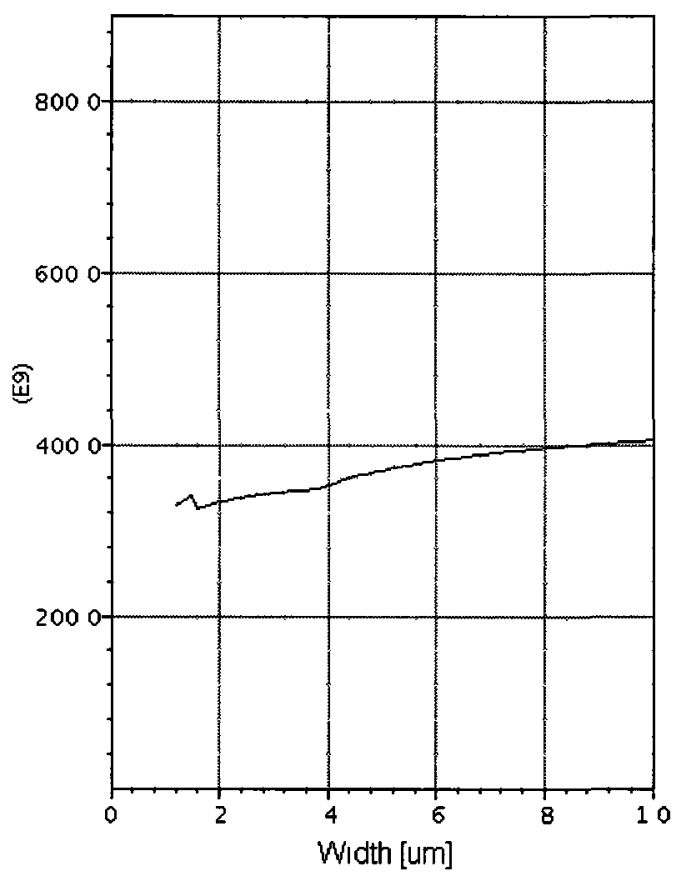

(b)

Figure 9: $65 \mathrm{~nm} L P, V_{D D}=1.2 \mathrm{~V}$; current-to-capacitance ratio $\left(I_{D} /\left(C_{G}+C_{S B}\right)\right)$ versus width for (a) PMOS and (b) NMOS 
Figure 10 plots the ratio for subthreshold. It is clear that the ratio is maximized at the minimum width of $0.12 \mu \mathrm{m}$ for both transistor types.

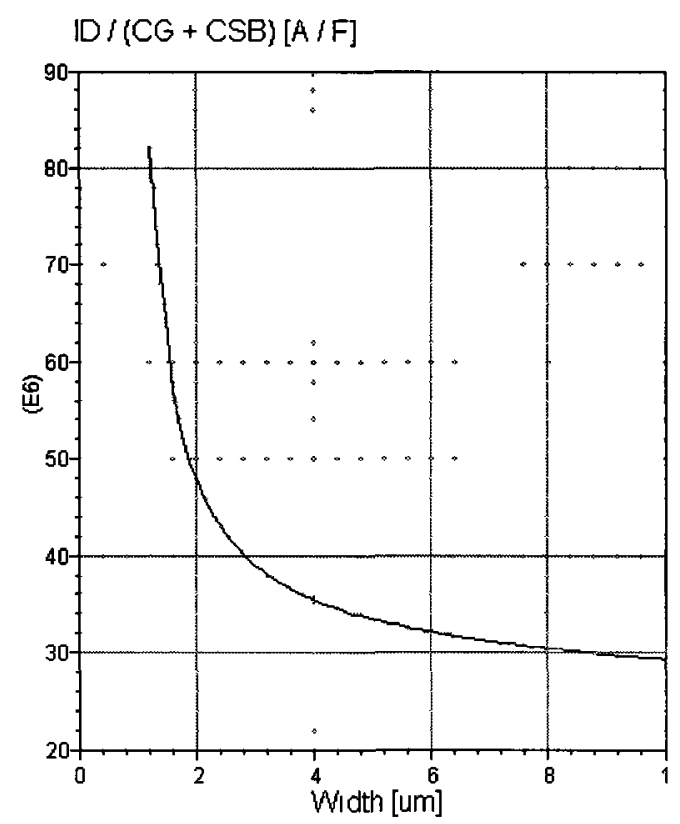

(a)

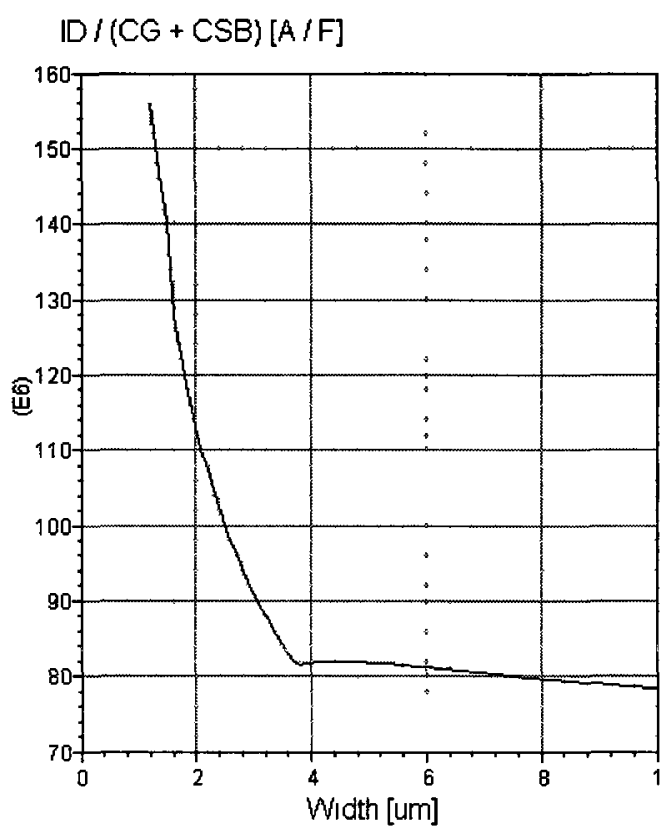

(b)

Figure 10: $65 \mathrm{~nm} \mathrm{LP}, V_{D D}=0.3 \mathrm{~V}$; current-to-capacitance ratio $\left(I_{D} /\left(C_{G}+C_{S B}\right)\right)$ versus width for (a) PMOS and (b) NMOS 
Figures 11 and 12 plot the $65 \mathrm{~nm}$ GP superthreshold and subthreshold current-tocapacitance ratios. They are similar to the $65 \mathrm{~nm}$ LP case, except that the optimum width in subthreshold for the NMOS transistor is slightly larger than minimum at $0.15 \mu \mathrm{m}$.

$\mathrm{DD} /(\mathrm{CG}+\mathrm{CSB})[\mathrm{A} / \mathrm{F}]$

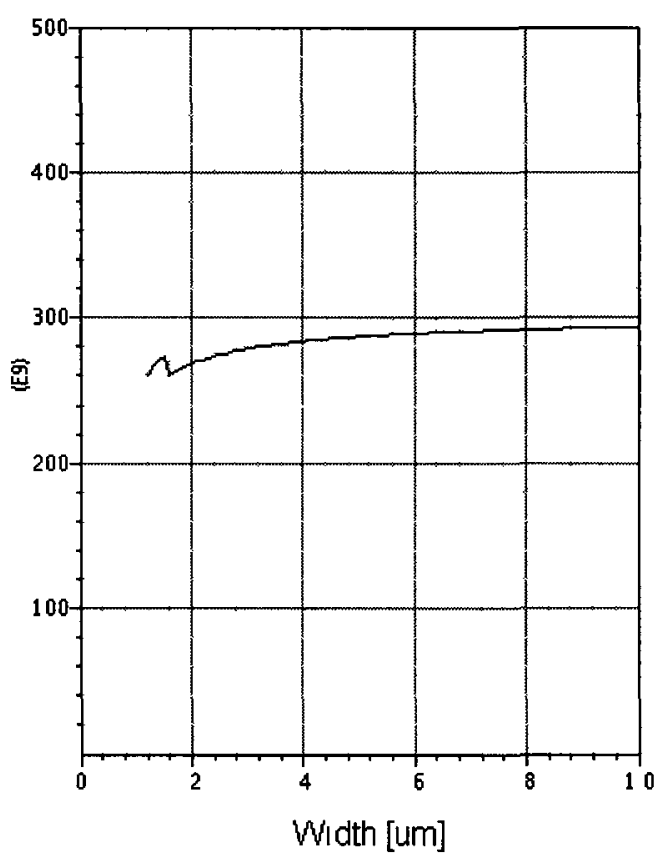

(a)
$\mathrm{DD} /(\mathrm{CG}+\mathrm{CSB})[\mathrm{A} / \mathrm{F}]$

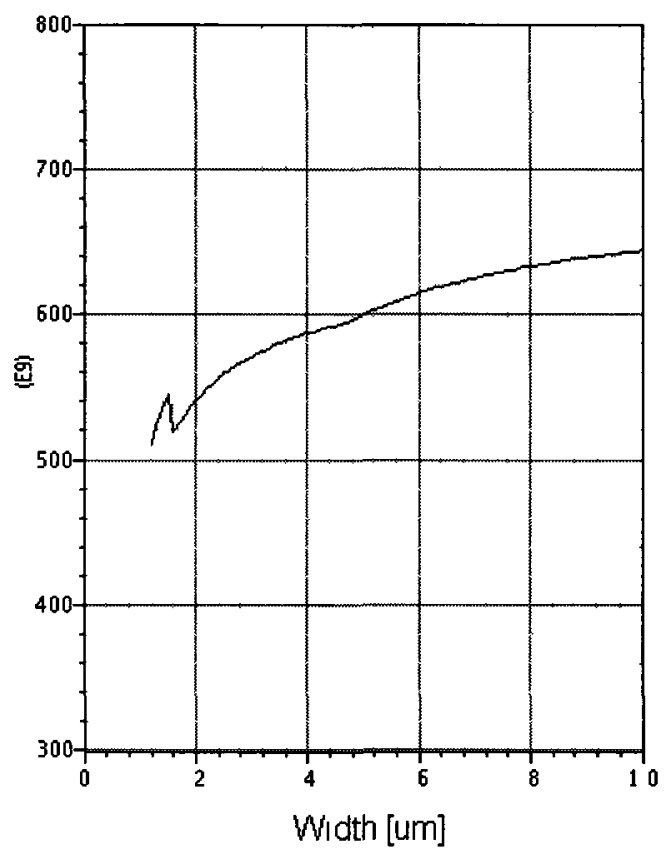

(b)

Figure 11: $65 \mathrm{~nm}$ GP, $V_{D D}=1.0 \mathrm{~V}$; current-to-capacitance ratio $\left(I_{D} /\left(C_{G}+C_{S B}\right)\right)$ versus width for (a) PMOS and (b) NMOS 
$\mathrm{ID} /\{\mathrm{CG}+\mathrm{CSB}\}[\mathrm{A} / \mathrm{F}]$

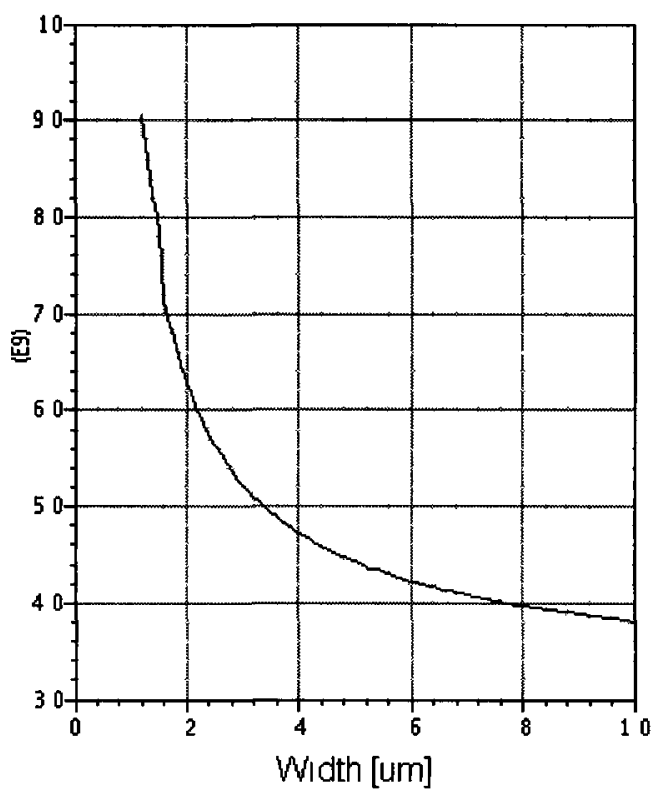

(a)
$\mathbb{D D} /(\mathrm{CG}+\mathrm{CSB})[\mathrm{A} / \mathrm{F}]$

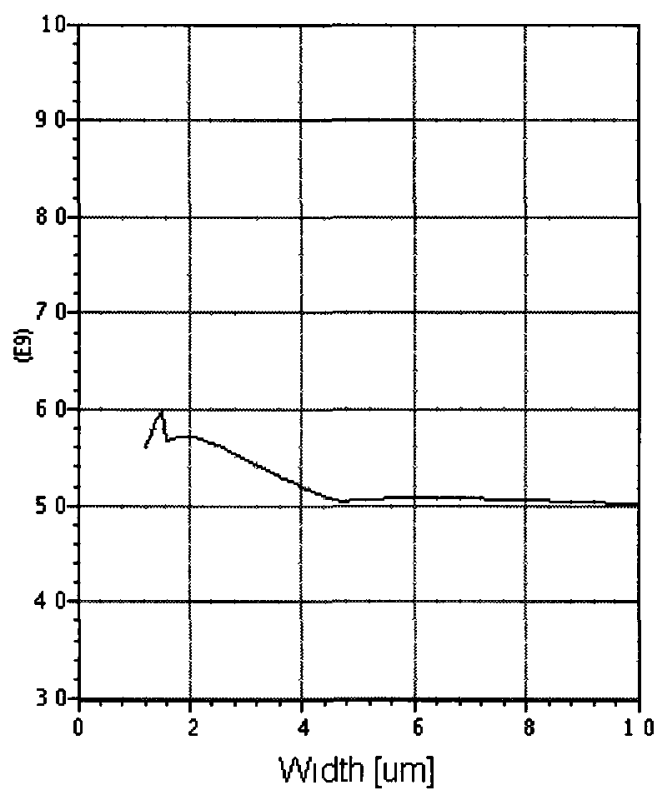

(b)

Figure 12: $65 \mathrm{~nm}$ GP, $V_{D D}=0.3 \mathrm{~V}$; current-to-capacitance ratio $\left(I_{D} /\left(C_{G}+C_{S B}\right)\right)$ versus width for (a) PMOS and (b) NMOS 
The $90 \mathrm{~nm}$ superthreshold current-to-capacitance ratios are shown in Figure 13. Again, they do not reach a maximum at less than $1 \mu \mathrm{m}$. Figure 14 shows how the $90 \mathrm{~nm}$ kit differs from both $65 \mathrm{~nm}$ kits as the PMOS transistor does not appear to have a subthreshold optimum width at less than $1 \mu \mathrm{m}$. The NMOS transistor is optimized at the minimum width of $0.12 \mu \mathrm{m}$.

In the next section, ring oscillator circuits will be used for confirming the subthreshold optimum widths found in a circuit context where both PMOS and NMOS transistors are employed together. Also, it will lead to finding a suitable width to use for the $90 \mathrm{~nm}$ PMOS transistor.

$\mathrm{DD} /(\mathrm{CG}+\mathrm{CSB})[\mathrm{A} / \mathrm{F}]$

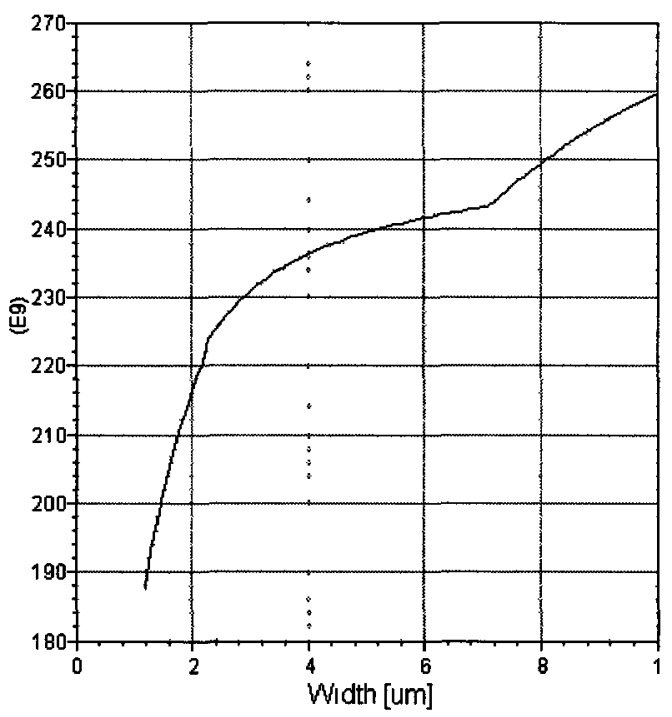

(a)

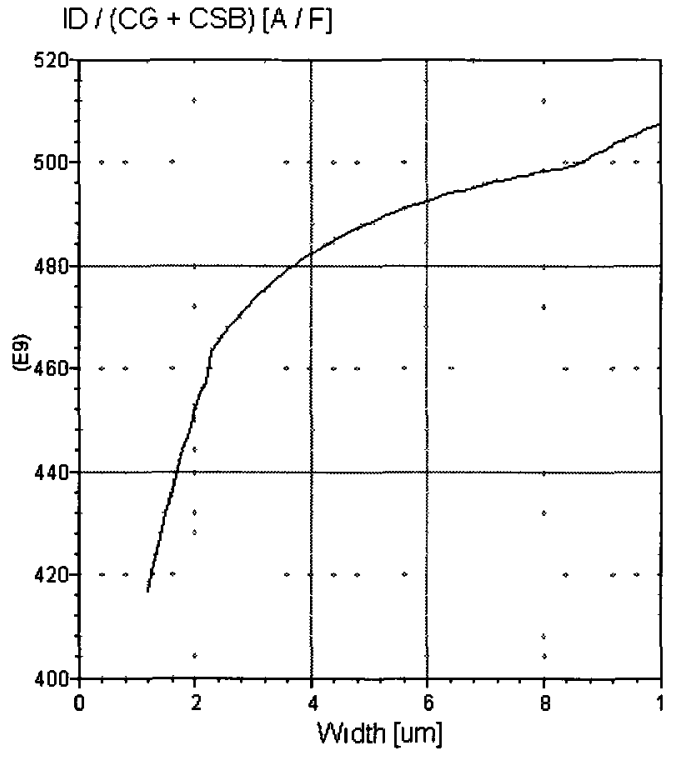

(b)

Figure 13: $90 \mathrm{~nm}, V_{D D}=1.2 \mathrm{~V}$; current-to-capacitance ratio $\left(I_{D} /\left(C_{G}+C_{S B}\right)\right)$ versus width for (a) PMOS and (b) NMOS 


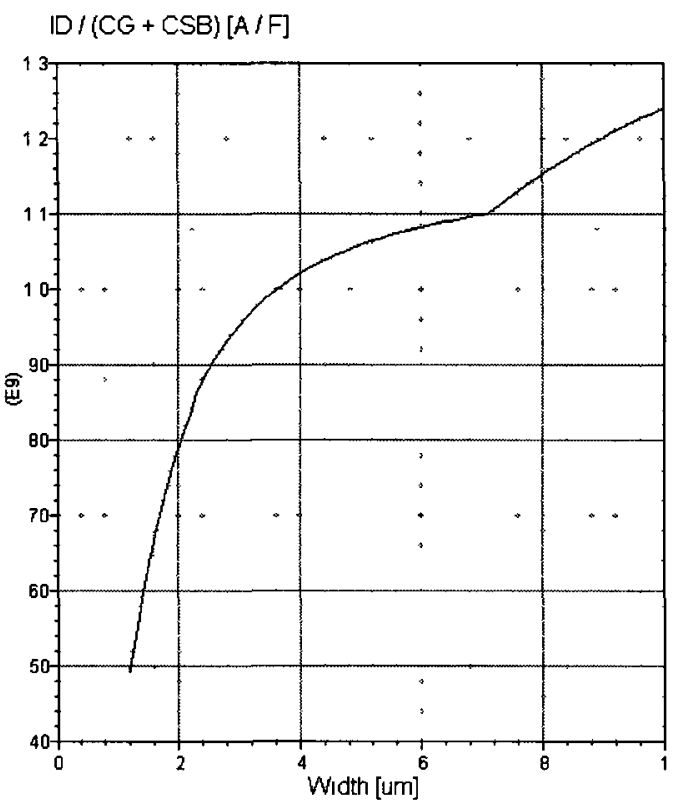

(a)

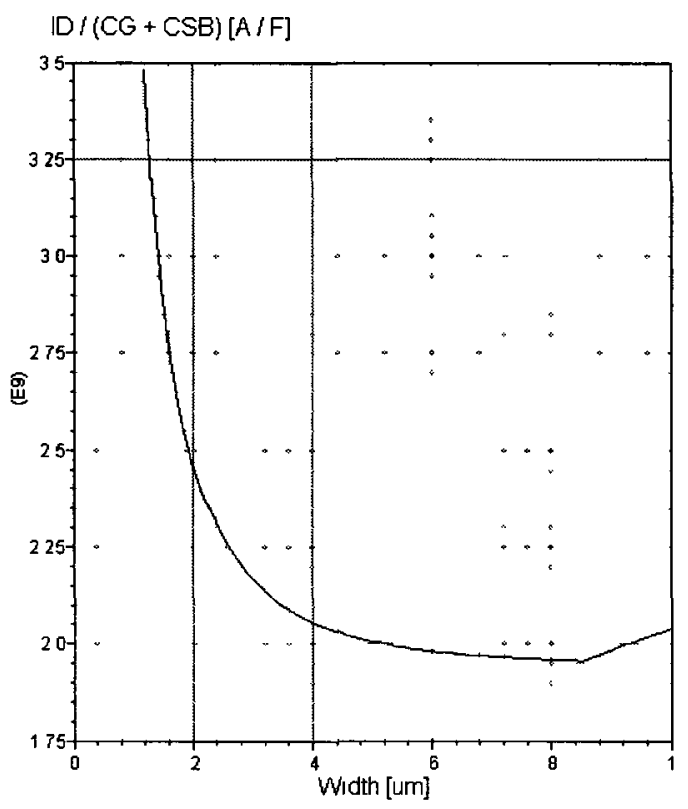

(b)

Figure 14: $90 \mathrm{~nm}, V_{D D}=0.3 \mathrm{~V}$; current-to-capacitance ratio $\left(I_{D} /\left(C_{G}+C_{S B}\right)\right)$ versus width for (a) PMOS and (b) NMOS

\subsection{Subthreshold Optimum Width from Ring Oscillator Circuit}

Ring oscillator circuits are the standard circuits used for comparing the performance of different technologies or circuit styles. They allow for a comparison that isn't affected by parameters such as fan-in and fan-out [15]. From Equation (11), the propagation delay can be seen to be a function of the transistor current and the load capacitance. Both the current and the load capacitance, composed of the gate and drain diffusion capacitances shown in Equations (4) and (7), scale almost linearly with the width. Thus, ideally, if the transistor widths of the inverters are scaled in a ring 
oscillator, any increase in current will be balanced by an increase in capacitance, resulting in identical propagation delays.

The reality is that there are higher order effects that will lead to widths that maximize the oscillation frequency. In superthreshold, the variation in performance between different widths may not be very large. However, given the subthreshold current-to-capacitance ratio results from the preceding section, it would be expected that the highest frequencies are obtained when using the widths that maximized the ratio.

It is not necessary for the inverters in the ring oscillator to have the same width for the PMOS and NMOS transistors. In fact, for a symmetric response in an inverter's output, the PMOS transistor will typically be two to three times wider than the NMOS to compensate for the reduced mobility of holes compared to electrons. The ratio of width between the PMOS and NMOS transistors is called the beta ratio. But if symmetry is not required, a better performance may be achieved from a smaller beta ratio [15].

In order to find the transistor widths that optimize the performance, the oscillation frequency of a ring oscillator circuit consisting of nine inverters is simulated. In Figure 15 , the oscillation frequency is plotted against the PMOS width for a fixed NMOS width of $0.12 \mu \mathrm{m}$ for the $65 \mathrm{~nm}$ LP technology at $1.2 \mathrm{~V}$. There are two local maximums, with the highest frequency of approximately $4.03 \mathrm{GHz}$ occurring at a PMOS width of $0.15 \mu \mathrm{m}$. Figure 16 shows the frequency as a function of the PMOS and NMOS widths. The frequency falls away as the difference in width between the two transistor types grows. 
Frequency $[\mathrm{Hz}]$

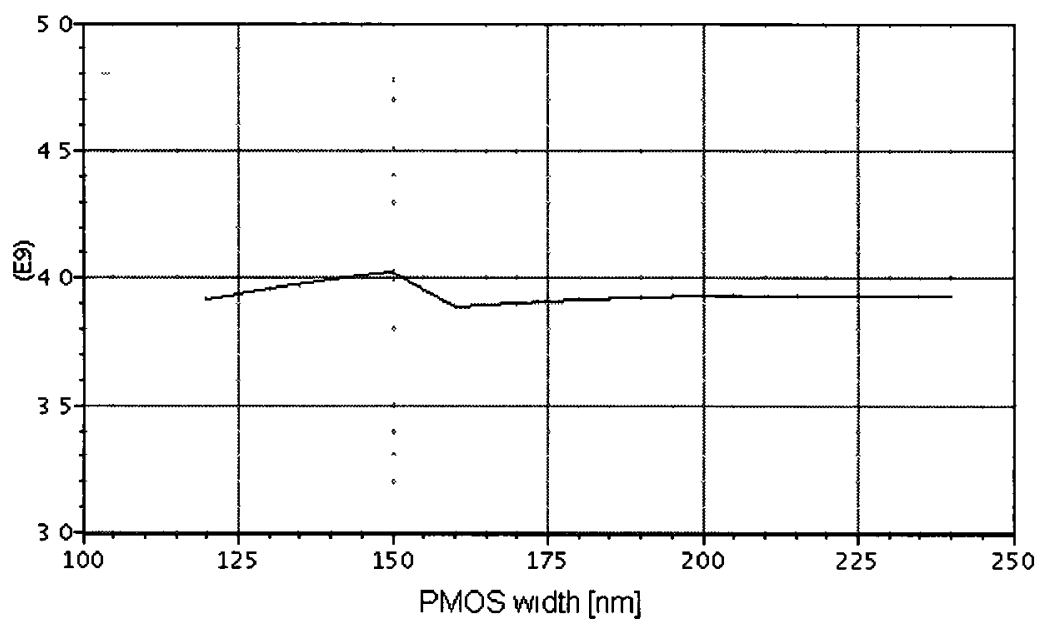

Figure 15: $65 \mathrm{~nm} \mathrm{LP}, V_{D D}=1.2 \mathrm{~V}$; 9-inverter ring oscillator frequency versus PMOS width for $0.12 \mu \mathrm{m} N M O S$ width

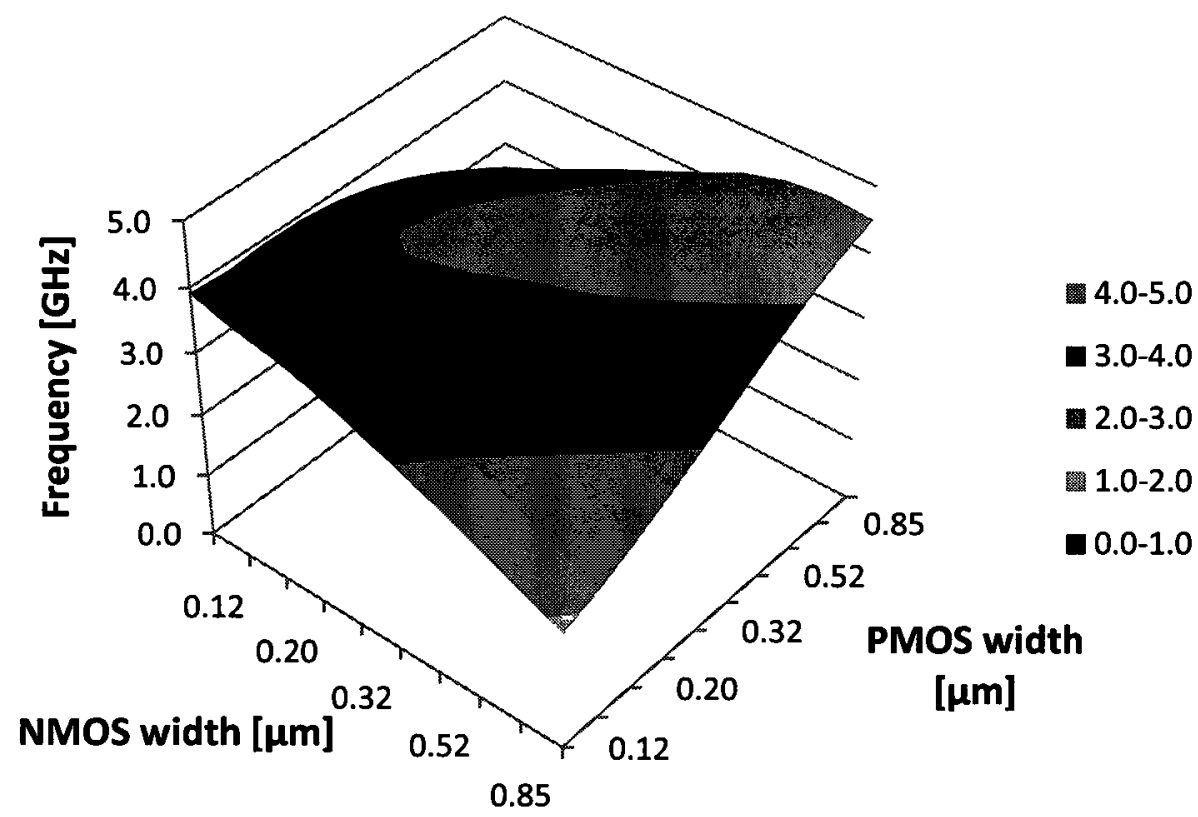

Figure 16: $65 \mathrm{~nm} \mathrm{LP}, \mathrm{V}_{\mathrm{DD}}=1.2 \mathrm{~V}$; 9-inverter ring oscillator frequency as a function of PMOS and NMOS width 
The maximum frequencies for each NMOS width simulated are summarized in

Table 3. The corresponding PMOS widths and beta ratios are included as well. The widest NMOS transistor simulated was $1.08 \mu \mathrm{m}$, which is the width at which the highest frequency occurred for the data obtained. Had wider transistors been simulated, marginally higher frequencies may have been obtained. Regardless, working within the data presented, the drop-off in performance from the highest frequency obtained to the lowest is $13.9 \%$.

Table 3: $65 \mathrm{~nm} \mathrm{LP,} V_{D D}=1.2 \mathrm{~V}$; 9-inverter ring oscillator maximum frequencies for varying NMOS widths

\begin{tabular}{|c|c|c|c|}
\hline $\mathbf{W}_{\mathbf{n}}[\boldsymbol{\mu m}]$ & $\mathbf{f}_{\max }[\mathbf{G H z}]$ & $\mathbf{W}_{\mathbf{p}, \mathbf{p p t}}[\boldsymbol{\mu m}]$ & $\boldsymbol{\beta}_{\mathbf{o p t}}$ \\
\hline 0.12 & 4.028 & 0.15 & 1.25 \\
\hline 0.13 & 4.039 & 0.15 & 1.15 \\
\hline 0.14 & 4.042 & 0.15 & 1.07 \\
\hline 0.15 & 4.040 & 0.15 & 1.00 \\
\hline 0.18 & 3.990 & 0.31 & 1.72 \\
\hline 0.21 & 4.040 & 0.35 & 1.67 \\
\hline 0.24 & 4.080 & 0.39 & 1.63 \\
\hline 0.36 & 4.179 & 0.55 & 1.53 \\
\hline 0.48 & 4.337 & 0.73 & 1.52 \\
\hline 0.60 & 4.443 & 0.90 & 1.50 \\
\hline 0.72 & 4.514 & 1.08 & 1.50 \\
\hline 0.84 & 4.565 & 1.26 & 1.50 \\
\hline 0.96 & 4.603 & 1.43 & 1.49 \\
\hline 1.08 & 4.633 & 1.61 & 1.49 \\
\hline
\end{tabular}

The subthreshold $65 \mathrm{~nm}$ LP oscillation frequency for a fixed $0.12 \mu \mathrm{m}$ NMOS width is shown in Figure 17. The frequency monotonically decreases with increasing PMOS width. Also shown in Figure 18 is the plot of frequency for a fixed NMOS width 
of $1.08 \mu \mathrm{m}$. It can be seen that even with such a wide NMOS transistor, the highest frequency occurs for a minimum-sized PMOS, although the presence of a second maximum around a width of $1.5 \mu \mathrm{m}$ is evident. In Figure 19, the frequency is plotted as a function of PMOS and NMOS width. The frequency is maximized when both transistors are sized at the minimum width of $0.12 \mu \mathrm{m}$, and falls away as the widths are increased.

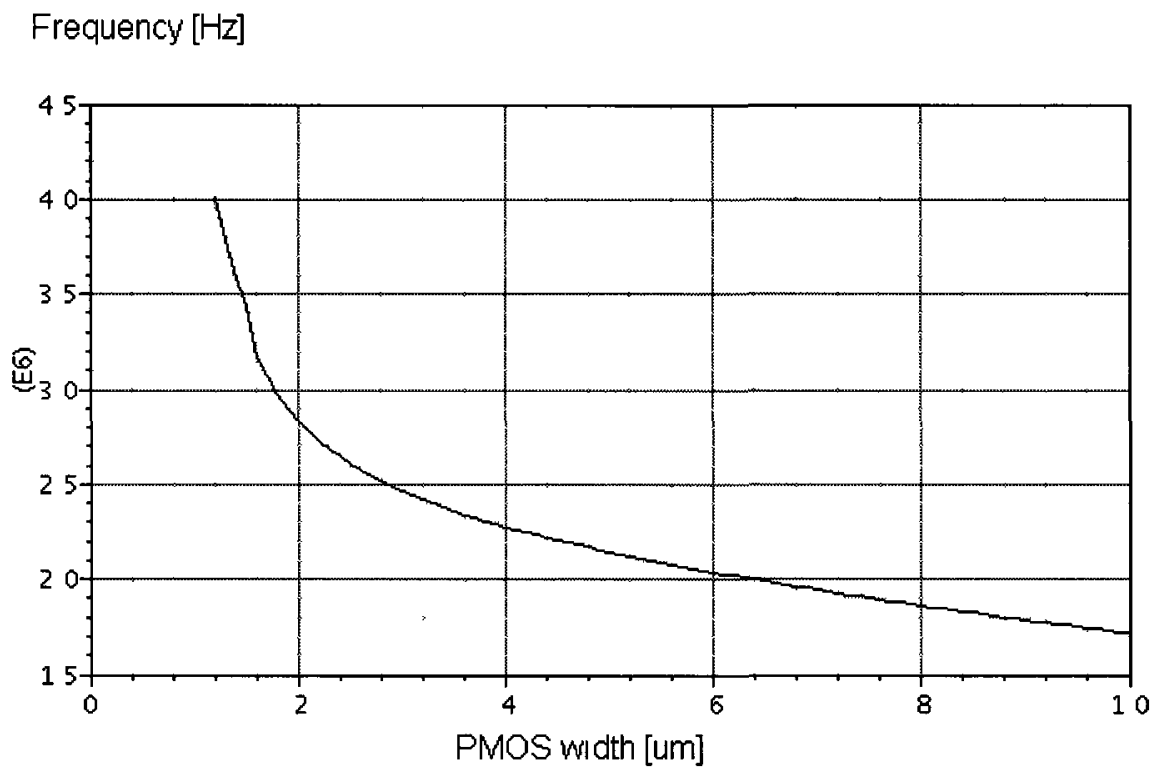

Figure 17: $65 \mathrm{~nm} \mathrm{LP}, V_{D D}=0.3 \mathrm{~V}$; 9-inverter ring oscillator frequency versus PMOS width for $0.12 \mu \mathrm{m}$ NMOS width 
Frequency $[\mathrm{Hz}]$

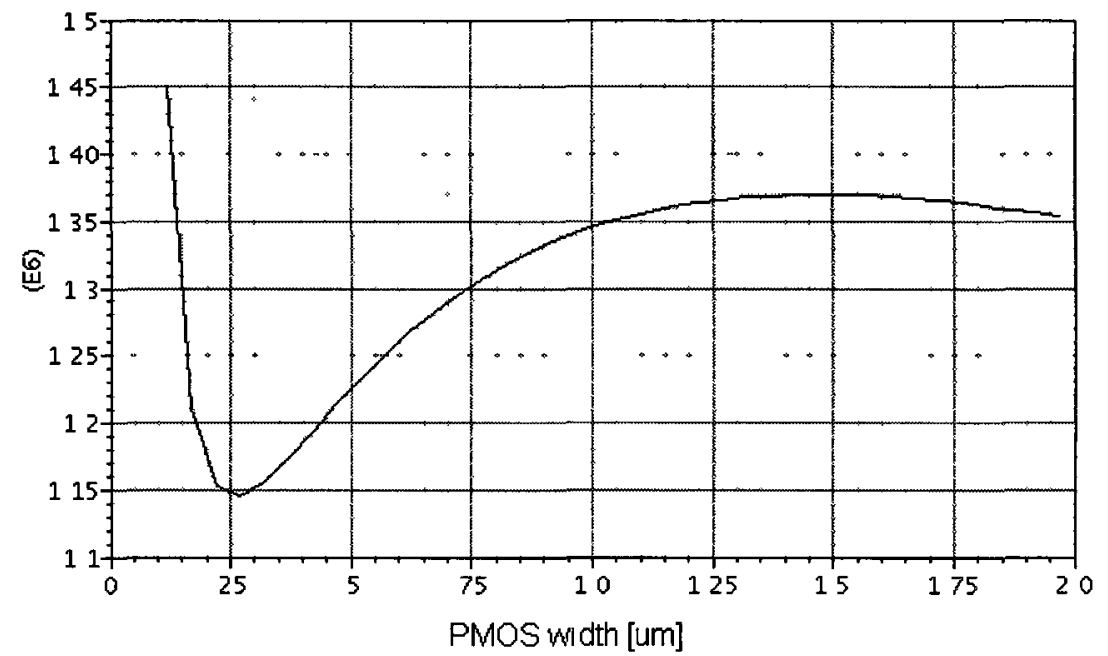

Figure 18: $65 \mathrm{~nm} \mathrm{LP}, V_{D D}=0.3 \mathrm{~V}$; 9-inverter ring oscillator frequency versus PMOS width for $1.08 \mu \mathrm{m}$ NMOS width

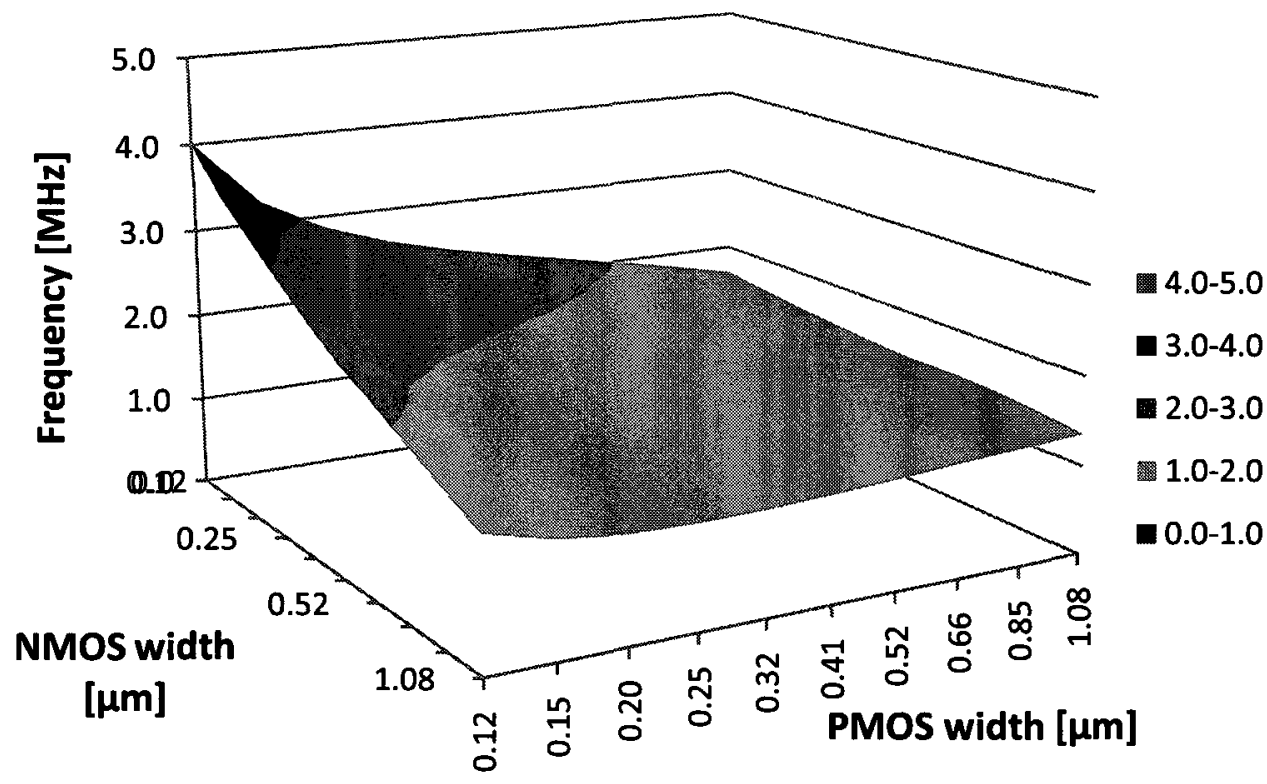

Figure 19: $65 \mathrm{~nm} \mathrm{LP}, \mathrm{V}_{\mathrm{DD}}=0.3 \mathrm{~V}$; 9-inverter ring oscillator frequency as a function of PMOS and NMOS width 
Table 4 summarizes the subthreshold results. The best performance occurs when both transistors are at the minimum width of $0.12 \mu \mathrm{m}$. This matches the subthreshold optimum widths found in the preceding section from the current-to-capacitance ratio when each transistor type was considered individually. Although the subthreshold optimum widths are in agreement here, should a discrepancy between the two methods result, priority should be given to the ring oscillator results since they are obtained in a method that is more in tune with a circuit design context.

Table 4: $65 \mathrm{~nm} \mathrm{LP,} V_{D D}=0.3 \mathrm{~V}$; 9-inverter ring oscillator maximum frequencies for varying NMOS widths

\begin{tabular}{|c|c|c|c|}
\hline $\mathbf{W}_{\mathbf{n}}[\boldsymbol{\mu m}]$ & $\mathbf{f}_{\mathbf{m a x}}[\mathbf{M H z}]$ & $\mathbf{W}_{\mathbf{p}, \mathbf{p p t}}[\boldsymbol{\mu m}]$ & $\boldsymbol{\beta}_{\mathbf{~ o p t}}$ \\
\hline $\mathbf{0 . 1 2}$ & $\mathbf{4 . 0 1 2}$ & $\mathbf{0 . 1 2}$ & $\mathbf{1 . 0 0}$ \\
\hline 0.13 & 3.917 & 0.12 & 0.92 \\
\hline 0.14 & 3.827 & 0.12 & 0.86 \\
\hline 0.15 & 3.740 & 0.12 & 0.80 \\
\hline 0.18 & 3.343 & 0.12 & 0.67 \\
\hline 0.21 & 3.139 & 0.12 & 0.57 \\
\hline 0.24 & 2.965 & 0.12 & 0.50 \\
\hline 0.36 & 2.458 & 0.12 & 0.33 \\
\hline 0.48 & 2.201 & 0.12 & 0.25 \\
\hline 0.60 & 1.994 & 0.12 & 0.20 \\
\hline 0.72 & 1.821 & 0.12 & 0.17 \\
\hline 0.84 & 1.667 & 0.12 & 0.14 \\
\hline 0.96 & 1.555 & 0.12 & 0.13 \\
\hline 1.08 & 1.450 & 0.12 & 0.11 \\
\hline
\end{tabular}

The drop-off from the highest to lowest frequency within the dataset of Table 4 is $63.9 \%$ compared to $13.9 \%$ of the superthreshold case. Thus it is evident that in subthreshold, there may be a huge penalty in performance if the correct widths are not 
used. Coupled with the knowledge that larger transistor sizes result in more energy consumption due to the increase in capacitance, it becomes clear that in the $65 \mathrm{~nm} \mathrm{LP}$ technology kit, there is no justification for using larger transistors.

Post-layout simulations (PLS) were also performed to verify that the subthreshold optimum widths obtained from the schematic simulations are also valid when all the parasitics of a real circuit are considered. A sample 9-inverter ring oscillator layout is shown in Figure 20. Table 5 contains the post-layout simulation oscillation frequencies as well as those from schematic simulations. The post-layout simulation frequencies range from 38.0 to $49.2 \%$ slower compared to those of the schematic simulations, and the results generally follow the same trends. Most importantly, the subthreshold optimum width of $0.12 \mu \mathrm{m}$ for both PMOS and NMOS transistors is confirmed.

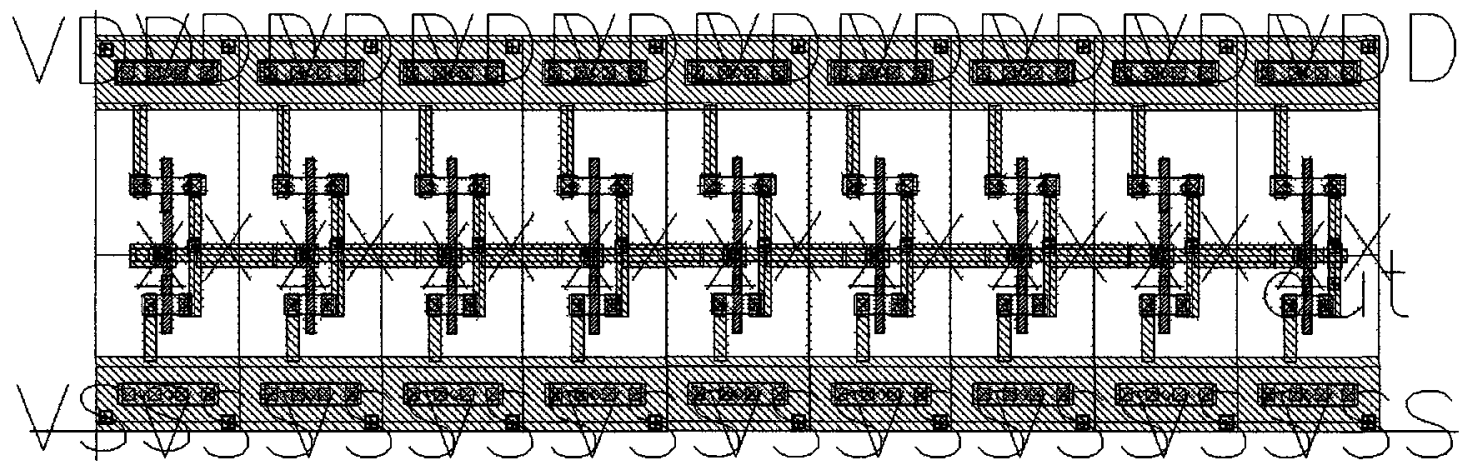

Figure 20: 9-inverter ring oscillator layout where each inverter consists of one $0.15 \mu \mathrm{m}$ width PMOS and one $0.12 \mu \mathrm{m}$ width NMOS 
Table 5: $65 \mathrm{~nm} \mathrm{LP}, V_{D D}=0.3 \mathrm{~V}$; 9-inverter ring oscillator schematic and post-layout simulation frequencies

\begin{tabular}{|c|c|c|c|}
\hline $\mathbf{W}_{\mathbf{p}}[\boldsymbol{\mu m}]$ & $\mathbf{W}_{\mathbf{n}}[\boldsymbol{\mu m}]$ & $\mathbf{f}$-schematic $[\mathbf{M H z}]$ & $\mathbf{f}-\mathbf{P L S}[\mathbf{M H z}]$ \\
\hline 0.12 & 0.12 & 4.012 & 2.355 \\
\hline 0.12 & 0.15 & 3.740 & 2.227 \\
\hline 0.12 & 0.18 & 3.343 & 2.071 \\
\hline 0.12 & 0.21 & 3.139 & 1.908 \\
\hline 0.15 & 0.12 & 3.441 & 1.748 \\
\hline 0.15 & 0.15 & 3.221 & 1.664 \\
\hline 0.18 & 0.12 & 2.977 & 1.709 \\
\hline 0.18 & 0.18 & 2.546 & 1.524 \\
\hline 0.21 & 0.12 & 2.780 & 1.670 \\
\hline 0.21 & 0.21 & 2.277 & 1.389 \\
\hline
\end{tabular}

In Table 6, the $65 \mathrm{~nm}$ GP superthreshold maximum frequencies are summarized. For NMOS widths between 0.12 and $1.08 \mu \mathrm{m}$, the best width $1 \mathrm{~s} 0.84 \mu \mathrm{m}$. There is a $9.0 \%$ drop-off in performance from the highest to lowest frequency. Table 7 contains the subthreshold results. As with the current-to-capacitance ratios, the subthreshold optimum widths are $0.12 \mu \mathrm{m}$ for PMOS and $0.15 \mu \mathrm{m}$ for NMOS. The performance dropoff is $47.5 \%$ from highest to lowest frequency. 
Table 6: $65 \mathrm{~nm} \mathrm{GP,} V_{D D}=1 \mathrm{~V}$; 9-inverter ring oscillator maximum frequencies for varying NMOS widths

\begin{tabular}{|c|c|c|c|}
\hline $\mathbf{W}_{\mathbf{n}}[\boldsymbol{\mu m}]$ & $\mathbf{f}_{\max }[\mathbf{G H z}]$ & $\mathbf{W}_{\mathbf{p}, \mathbf{o p t}}[\boldsymbol{\mu m}]$ & $\boldsymbol{\beta}_{\mathbf{o p t}}$ \\
\hline 0.12 & 7.445 & 0.15 & 1.25 \\
\hline 0.13 & 7.513 & 0.15 & 1.15 \\
\hline 0.14 & 7.561 & 0.15 & 1.07 \\
\hline 0.15 & 7.593 & 0.15 & 1.00 \\
\hline 0.18 & 7.500 & 0.30 & 1.67 \\
\hline 0.21 & 7.624 & 0.34 & 1.62 \\
\hline 0.24 & 7.715 & 0.38 & 1.58 \\
\hline 0.36 & 7.913 & 0.54 & 1.50 \\
\hline 0.48 & 8.006 & 0.70 & 1.46 \\
\hline 0.60 & 8.122 & 0.86 & 1.43 \\
\hline 0.72 & 8.176 & 0.95 & 1.32 \\
\hline $\mathbf{0 . 8 4}$ & $\mathbf{8 . 1 8 0}$ & $\mathbf{1 . 1 0}$ & $\mathbf{1 . 3 1}$ \\
\hline 0.96 & 8.172 & 1.25 & 1.30 \\
\hline 1.08 & 8.152 & 1.39 & 1.29 \\
\hline
\end{tabular}

Table 7: $65 \mathrm{~nm} \mathrm{GP,} V_{D D}=0.3 \mathrm{~V}$; 9-inverter ring oscillator maximum frequencies for varying NMOS widths

\begin{tabular}{|c|c|c|c|}
\hline $\mathbf{W}_{\mathbf{n}}[\boldsymbol{\mu m}]$ & $\mathbf{f}_{\mathbf{m a x}}[\mathbf{M H z}]$ & $\mathbf{W}_{\mathbf{p}, \mathbf{p p t}}[\boldsymbol{\mu m}]$ & $\boldsymbol{\beta}_{\mathbf{p p t}}$ \\
\hline 0.12 & 315.8 & 0.12 & 1.00 \\
\hline 0.13 & 322.1 & 0.12 & 0.92 \\
\hline 0.14 & 326.5 & 0.12 & 0.86 \\
\hline $\mathbf{0 . 1 5}$ & 329.6 & $\mathbf{0 . 1 2}$ & $\mathbf{0 . 8 0}$ \\
\hline 0.16 & 315.9 & 0.12 & 0.75 \\
\hline 0.18 & 315.6 & 0.12 & 0.67 \\
\hline 0.21 & 311.6 & 0.12 & 0.57 \\
\hline 0.24 & 305.2 & 0.12 & 0.50 \\
\hline 0.36 & 274.4 & 0.12 & 0.33 \\
\hline 0.48 & 247.5 & 0.12 & 0.25 \\
\hline 0.60 & 229.0 & 0.12 & 0.20 \\
\hline 0.72 & 212.1 & 0.12 & 0.17 \\
\hline 0.84 & 197.3 & 0.12 & 0.14 \\
\hline 0.96 & 184.4 & 0.12 & 0.13 \\
\hline 1.08 & 173.0 & 0.12 & 0.11 \\
\hline
\end{tabular}


For the $90 \mathrm{~nm}$ superthreshold results, Table 8 shows that the highest frequency for the widths simulated occurs at an NMOS width of $1.08 \mu \mathrm{m}$. The drop-off from highest to lowest frequency is $19.8 \%$. Table 9 contains the subthreshold frequencies. Recall from the preceding section that the subthreshold optimum width for the NMOS transistor was determined to be $0.12 \mu \mathrm{m}$, while no apparent optimum width for the PMOS was found. However, with these ring oscillator results, a PMOS width of $0.41 \mu \mathrm{m}$ can be found that pairs with the $0.12 \mu \mathrm{m}$ NMOS transistor to give the best performance. The drop-off from highest to lowest frequency is only $18.5 \%$, similar to the superthreshold scenario. It is clear that unlike the two $65 \mathrm{~nm}$ technologies, any gains obtained from exploiting the inverse-narrow-width effect will not be as substantial in the $90 \mathrm{~nm}$ technology since only the NMOS transistor has this property.

Table 8: $90 \mathrm{~nm}, V_{D D}=1.2 \mathrm{~V}$; 9-inverter ring oscillator maximum frequencies for varying NMOS widths

\begin{tabular}{|c|c|c|c|}
\hline $\mathbf{W}_{\mathbf{n}}[\boldsymbol{\mu m}]$ & $\mathbf{f}_{\max }[\mathbf{G H z}]$ & $\mathbf{W}_{\mathbf{p}, \mathbf{p p t}}[\boldsymbol{\mu m}]$ & $\boldsymbol{\beta}_{\mathbf{o p t}}$ \\
\hline 0.12 & 5.388 & 0.24 & 2.00 \\
\hline 0.13 & 5.438 & 0.25 & 1.92 \\
\hline 0.14 & 5.484 & 0.27 & 1.93 \\
\hline 0.15 & 5.527 & 0.28 & 1.87 \\
\hline 0.18 & 5.639 & 0.32 & 1.78 \\
\hline 0.21 & 5.728 & 0.36 & 1.71 \\
\hline 0.24 & 5.813 & 0.40 & 1.67 \\
\hline 0.36 & 5.969 & 0.57 & 1.58 \\
\hline 0.48 & 6.158 & 0.92 & 1.92 \\
\hline 0.60 & 6.318 & 1.08 & 1.80 \\
\hline 0.72 & 6.433 & 1.23 & 1.71 \\
\hline 0.84 & 6.519 & 1.39 & 1.65 \\
\hline 0.96 & 6.627 & 1.55 & 1.61 \\
\hline $\mathbf{1 . 0 8}$ & $\mathbf{6 . 7 1 7}$ & $\mathbf{1 . 7 1}$ & $\mathbf{1 . 5 8}$ \\
\hline
\end{tabular}


Table 9: $90 \mathrm{~nm}, V_{D D}=0.3 \mathrm{~V}$; 9-inverter ring oscillator maximum frequencies for varying NMOS widths

\begin{tabular}{|c|c|c|c|}
\hline $\mathbf{W}_{\mathbf{n}}[\boldsymbol{\mu m}]$ & $\mathbf{f}_{\mathbf{m a x}}[\mathbf{M H z}]$ & $\mathbf{W}_{\mathbf{p}, \mathbf{p p t}}[\boldsymbol{\mu m}]$ & $\boldsymbol{\beta}_{\mathbf{p p t}}$ \\
\hline $\mathbf{0 . 1 2}$ & $\mathbf{7 0 . 7 3}$ & $\mathbf{0 . 4 1}$ & $\mathbf{3 . 4 2}$ \\
\hline 0.13 & 68.40 & 0.41 & 3.15 \\
\hline 0.14 & 66.58 & 0.42 & 3.00 \\
\hline 0.15 & 65.11 & 0.42 & 2.80 \\
\hline 0.18 & 62.12 & 0.44 & 2.44 \\
\hline 0.21 & 60.42 & 0.47 & 2.24 \\
\hline 0.24 & 59.52 & 0.49 & 2.04 \\
\hline 0.36 & 57.68 & 0.62 & 1.72 \\
\hline 0.48 & 58.93 & 0.95 & 1.98 \\
\hline 0.60 & 59.99 & 1.08 & 1.80 \\
\hline 0.72 & 60.90 & 1.22 & 1.69 \\
\hline 0.84 & 61.67 & 1.36 & 1.62 \\
\hline 0.96 & 63.30 & 1.51 & 1.57 \\
\hline 1.08 & 64.74 & 1.68 & 1.56 \\
\hline
\end{tabular}

The optimum widths for all technologies in both superthreshold and subthreshold are summarized in Table 10.

Table 10: Ring oscillator optimum widths

\begin{tabular}{|c|c|c|c|c|}
\hline \multirow{2}{*}{ Technology } & \multicolumn{2}{|c|}{ Superthreshold } & \multicolumn{2}{c|}{ Subthreshold } \\
\cline { 2 - 5 } & PMOS $[\mu \mathrm{m}]$ & NMOS $[\mu \mathrm{m}]$ & PMOS $[\mu \mathrm{m}]$ & NMOS $[\mu \mathrm{m}]$ \\
\hline $65 \mathrm{~nm}$ LP & 1.61 & 1.08 & 0.12 & 0.12 \\
\hline $65 \mathrm{~nm}$ GP & 1.10 & 0.84 & 0.12 & 0.15 \\
\hline $90 \mathrm{~nm}$ & 1.71 & 1.08 & 0.41 & 0.12 \\
\hline
\end{tabular}




\subsection{Parallel Transistor Ratio}

The subthreshold optimum widths have been found in the previous two sections. For the ring oscillator simulations, the inverters have consisted of one PMOS and one NMOS transistor. While this may be satisfactory in most cases, there will be times when a larger gate will be required to drive a large load. It has been made clear that scaling the transistor widths is not a good solution in subthreshold due to the resulting dramatic decrease in current-to-capacitance ratio. Instead, we suggest that transistors at the subthreshold optimum width should be used in parallel.

From Table 10, the best inverter for the $65 \mathrm{~nm} \mathrm{LP} \mathrm{technology} \mathrm{in} \mathrm{subthreshold}$ consisted of both the PMOS and NMOS being sized at the minimum of $0.12 \mu \mathrm{m}$, thus having a beta ratio of 1 . Now let us assume that an inverter of five times this size is required. Thus the inverter will have five PMOS transistors in parallel matched with five NMOS transistors in parallel. But is this the best solution?

A slight improvement can be made by finding a parallel transistor ratio, or the ratio of PMOS-to-NMOS transistors, to use. In Figure 21, ring oscillator frequencies for $65 \mathrm{~nm}$ LP $0.3 \mathrm{~V}$ operation are plotted again. This time, the width of the NMOS and PMOS transistors are fixed at $0.12 \mu \mathrm{m}$. Twenty NMOS transistors are placed in parallel (multiplicity of 20) while the PMOS multiplicity is swept. From the plot, it can be seen that 27 PMOS transistors gives the best frequency of $4.098 \mathrm{MHz}$. The resulting parallel transistor ratio is $27 / 20=1.35$. The increase in frequency from using a parallel transistor ratio of 1.35 instead of 1 is only $2.1 \%$ at the cost of a $2.35 / 2=1.175$ times, or $17.5 \%$, increase in energy consumption. 
Frequency $[\mathrm{H} \mathrm{Z}]$

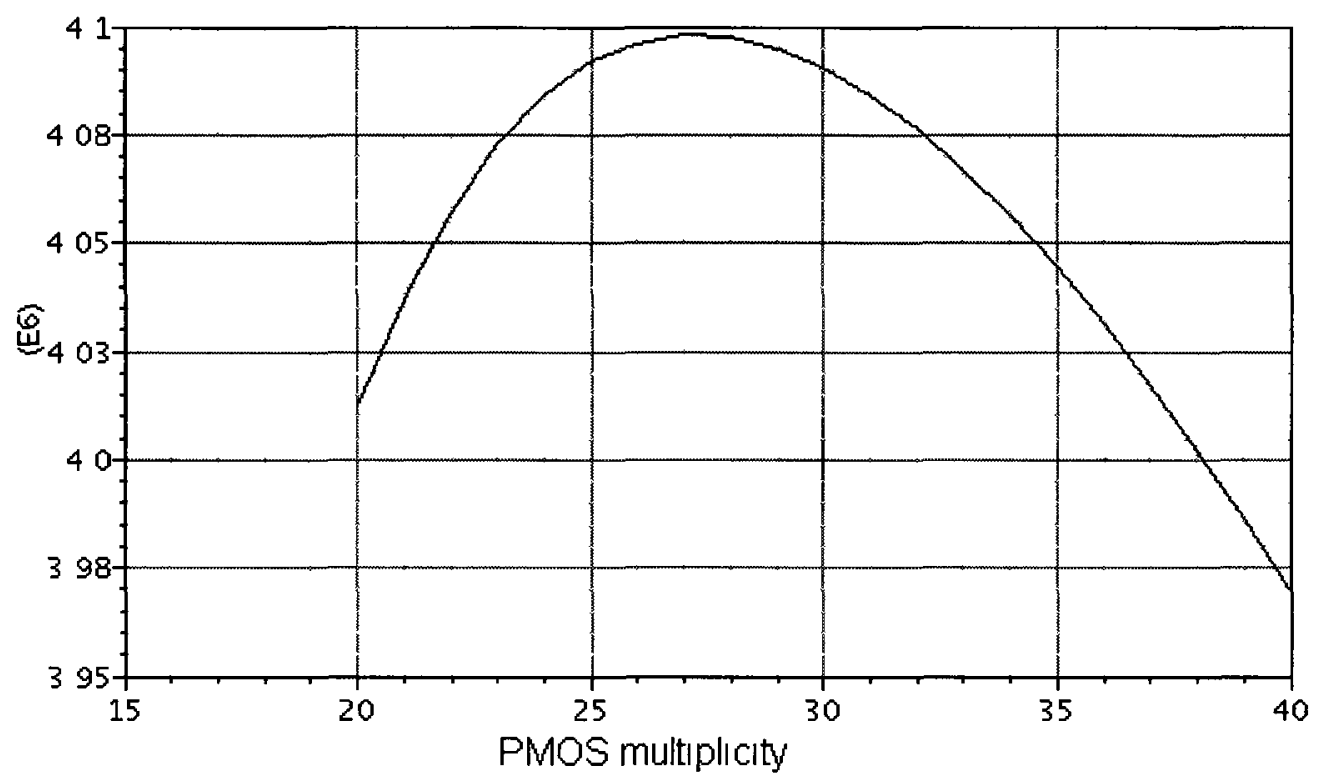

Figure 21: $65 \mathrm{~nm} \mathrm{LP}, V_{D D}=0.3 \mathrm{~V}$; 9-inverter ring oscillator frequency versus PMOS multiplicity for an NMOS multiplicity of 20

For the $65 \mathrm{~nm}$ GP kit, the optımum multiplicity of the PMOS transistor was 19 resulting in a frequency of $329.9 \mathrm{MHz}$. The parallel transistor ratio is thus 0.95 compared to the single transistor subthreshold optimum width ratio of 0.8 . For the $90 \mathrm{~nm} \mathrm{kit,} \mathrm{a}$ frequency of $72.17 \mathrm{MHz}$ was obtained with a PMOS multiplicity of 15 , resulting in a parallel transistor ratio of 0.75 compared to the single transistor subthreshold optimum width ratio of 3.42 . 


\subsection{Effective Beta Ratio}

Many sizing strategies such as Logical Effort reference the total input width, or sum of the PMOS and NMOS widths for a given input, in their calculations. Thus to simplify calculations, the subthreshold optimum widths $\left(W_{p, o p t}\right.$ and $\left.W_{n, o p t}\right)$ and the parallel transistor ratio (PTR) can be combined into an effective beta ratio $\left(\beta_{\text {eff }}\right)$ as

$$
\beta_{\text {eff }}=\frac{W_{p, o p t}}{W_{n, o p t}} \times P T R
$$

The effective beta ratio can also be described as the ratio of the total PMOS width to the total NMOS width. Now when using the sizing strategies, by employing the effective beta ratio, there is no need to consider the subthreshold optimum widths or the number of parallel transistors. Simply follow the same procedures as superthreshold where a transistor in a logic gate can have any width, with the only constraint being the beta ratio between the PMOS and NMOS transistors.

Table 11 summarizes the subthreshold optimum widths, the parallel transistor ratio and the effective beta ratio for the three technologies.

Table 11: Subthreshold optimum widths, parallel transistor ratios and effective beta ratios

\begin{tabular}{|c|c|c|c|c|c|}
\hline Technology & $\mathbf{W}_{\mathrm{p}, \mathbf{p p t}}[\boldsymbol{\mu m}]$ & $\mathbf{W}_{\mathrm{n}, \mathrm{opt}}[\boldsymbol{\mu m}]$ & $\boldsymbol{\beta}$ & $\mathbf{P T R}$ & $\boldsymbol{\beta}_{\text {eff }}$ \\
\hline $65 \mathrm{~nm}$ LP & 0.12 & 0.12 & 1 & 1.35 & 1.35 \\
\hline $65 \mathrm{~nm}$ GP & 0.12 & 0.15 & 0.8 & 0.95 & 0.76 \\
\hline $90 \mathrm{~nm}$ & 0.41 & 0.12 & 3.42 & 0.75 & 2.56 \\
\hline
\end{tabular}


As an example, suppose that the total width of an inverter is calculated to be 3.5 $\mu \mathrm{m}$ in the $90 \mathrm{~nm}$ technology. The PMOS portion of the width is $3.5 \mu \mathrm{m} \times(2.56 / 3.56)=$ $2.52 \mu \mathrm{m}$ while the NMOS portion is $3.5 \mu \mathrm{m} \times(1 / 3.56)=0.98 \mu \mathrm{m}$. The number of PMOS transistors is calculated as $2.52 \mu \mathrm{m} / 0.41 \mu \mathrm{m}=6$ while the number of NMOS is $0.98 \mu \mathrm{m} / 0.12 \mu \mathrm{m}=8$. The 6 PMOS and 8 NMOS transistors satisfy the parallel transistor ratio of 0.75 . This is illustrated in Figure 22 .

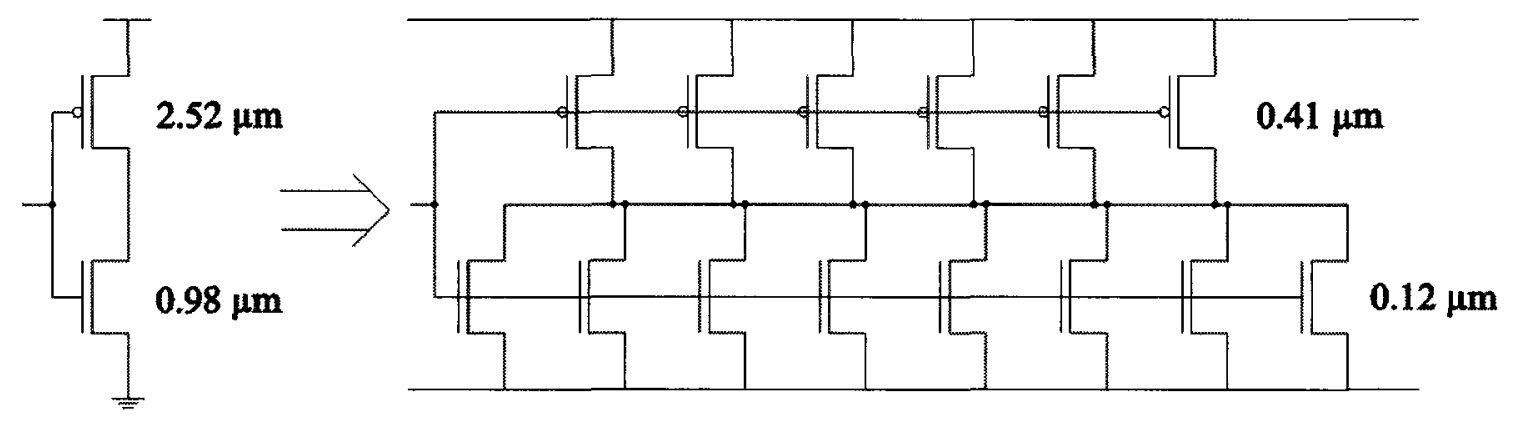

Figure 22: Inverter split into parallel transistors

\subsection{Transistor Stacks}

Logic circuits require more than just inverters. Gates such as NAND and NOR require the series connection of transistors. Typically, the widths of two transistors in series, or in a double stack, are doubled, while in the case of a triple stack, the widths are tripled. This approximates the current drive of a single transistor, although the specific scaling factor required is a function of factors such as the body effect and velocity saturation $[17,18]$. 
An equation developed in $[17,18]$ that describes the required scaled width when two transistors are in series is

$$
W=(1+\alpha) W_{e f f}
$$

where $W$ is the width of each transistor in the stack and $W_{\text {eff }}$ is the effective width if there was only a single transistor. The factor $\alpha$ is

$$
\alpha=e^{\frac{-\lambda_{d} V_{D D}}{m v_{T}}}
$$

where $\lambda_{d}$ is the drain-induced barrier lowering (DIBL) coefficient (a negative number), $m$ is an empirical parameter and $v_{T}$ is the thermal voltage. From $[17,18]$, the theoretical and simulated scaling factors from predictive technology models for $65 \mathrm{~nm} 0.3 \mathrm{~V}$ are 3.28 and 3.20 for PMOS and 2.93 and 2.64 for NMOS. While Equation (19) may be useful for larger transistors, it does not reflect the reality of narrow transistors in actual technology kits. This will be illustrated in the simulation results that follow.

In Table 12, the data for $65 \mathrm{~nm} \mathrm{LP}$ at $1.2 \mathrm{~V}$ is shown. The left side of the table displays the configuration (single, double stack or triple stack) of the transistors at the subthreshold optimum width with their corresponding current. The right side of the table displays the simulated scaled width required to achieve the current of the single transistor. Thus, for PMOS, the transistors have to be scaled by 2.42 and 3.92 times for double and triple stacks, while for NMOS, scaling factors of 2.08 and 3.25 are required. These values seem well within expectations. 
Table 12: $65 \mathrm{~nm} \mathrm{LP,} V_{D D}=1.2 \mathrm{~V}$; stack currents and scaled widths

\begin{tabular}{|c|c|c|c|}
\hline Configuration & $\mathbf{I}[\mu \mathrm{A}]$ & Scaled W $[\mu \mathrm{m}]$ & Scaled $\mathbf{I}[\boldsymbol{\mu A}]$ \\
\hline $1 \times 0.12 \mu \mathrm{m}$ PMOS & 48.34 & N/A & N/A \\
\hline $2 \times 0.12 \mu \mathrm{m}$ PMOS & 25.04 & $0.29(2.42 \mathrm{x})$ & 49.68 \\
\hline $3 \times 0.12 \mu \mathrm{m}$ PMOS & 16.56 & $0.47(3.92 \mathrm{x})$ & 49.17 \\
\hline $1 \times 0.12 \mu \mathrm{m}$ NMOS & 84.11 & N/A & N/A \\
\hline $2 \times 0.12 \mu \mathrm{m}$ NMOS & 47.65 & $0.25(2.08 \mathrm{x})$ & 85.86 \\
\hline $3 \times 0.12 \mu \mathrm{m}$ NMOS & 32.27 & $0.39(3.25 \mathrm{x})$ & 85.27 \\
\hline
\end{tabular}

Things are very different in subthreshold, as seen in Table 13. Here, a double stack of PMOS transistors must be scaled 16.83 times to achieve the current of a single transistor, while a triple stack requires a scaling factor of 30.75 . For NMOS, the scaling factors are 11.17 and 21.50 for double and triple stacks, respectively. These values are impractical to implement, and thus it can be concluded that should additional current drive be required from a stack of transistors, they should be implemented as parallel stacks. The simulated double stack scaling factors of 16.83 and 11.17 for PMOS and NMOS, respectively, are much higher than the scaling factors calculated using Equations (19) and (20): 2.455 and 4.776.

Table 13: $65 \mathrm{~nm} \mathrm{LP,} V_{D D}=0.3 \mathrm{~V}$; stack currents and scaled widths

\begin{tabular}{|c|c|c|c|}
\hline Configuration & I [nA] & Scaled W $[\mu \mathrm{m}]$ & Scaled I [nA] \\
\hline $1 \times 0.12 \mu \mathrm{m}$ PMOS & 18.74 & N/A & N/A \\
\hline $2 \times 0.12 \mu \mathrm{m}$ PMOS & 6.299 & $2.02(16.83 \times)$ & 18.80 \\
\hline $3 \times 0.12 \mu \mathrm{m}$ PMOS & 3.822 & $3.69(30.75 \mathrm{x})$ & 18.77 \\
\hline $1 \times 0.12 \mu \mathrm{m}$ NMOS & 36.32 & N/A & N/A \\
\hline $2 \times 0.12 \mu \mathrm{m}$ NMOS & 12.24 & $1.34(11.17 \times)$ & 36.41 \\
\hline $3 \times 0.12 \mu \mathrm{m}$ NMOS & 7.34 & $2.58(21.50 \mathrm{x})$ & 36.40 \\
\hline
\end{tabular}


The data for $65 \mathrm{~nm}$ GP is shown in Tables 14 and 15. While the scaling factors in subthreshold are less extreme than those of the $65 \mathrm{~nm}$ LP technology, they are still large enough to be impractical. The data for $90 \mathrm{~nm}$ is shown in Tables 16 and 17 . The thing to note here is that the subthreshold scaling factors for the PMOS transistor are reasonable, right in line with those of superthreshold. This is not surprising since the subthreshold optimum width is relatively large and as we have already seen from Figure 8 that the PMOS transistor displays narrow-width effect and thus does not have a larger than expected current at the narrower widths.

Table 14: $65 \mathrm{~nm}$ GP, $V_{D D}=1 \mathrm{~V}$; stack currents and scaled widths

\begin{tabular}{|c|c|c|c|}
\hline Configuration & $\mathbf{I}[\boldsymbol{\mu A}]$ & Scaled W $[\boldsymbol{\mu m}]$ & Scaled I $[\boldsymbol{\mu A}]$ \\
\hline $1 \times 0.12 \mu \mathrm{m}$ PMOS & 60.42 & N/A & N/A \\
\hline $2 \times 0.12 \mu \mathrm{m}$ PMOS & 31.75 & $0.28(2.33 \mathrm{x})$ & 60.51 \\
\hline $3 \times 0.12 \mu \mathrm{m}$ PMOS & 21.26 & $0.46(3.83 \mathrm{x})$ & 61.06 \\
\hline $1 \times 0.15 \mu \mathrm{m}$ NMOS & 130.8 & N/A & N/A \\
\hline $2 \times 0.15 \mu \mathrm{m}$ NMOS & 72.97 & $0.29(1.93 \mathrm{x})$ & 131.2 \\
\hline $3 \times 0.15 \mu \mathrm{m}$ NMOS & 50.14 & $0.45(3.00 \mathrm{x})$ & 133.1 \\
\hline
\end{tabular}

Table 15: $65 \mathrm{~nm}$ GP, $V_{D D}=0.3 \mathrm{~V}$; stack currents and scaled widths

\begin{tabular}{|c|c|c|c|}
\hline Configuration & I [nA] & Scaled W $[\mu \mathbf{m}]$ & Scaled I [nA] \\
\hline $1 \times 0.12 \mu \mathrm{m}$ PMOS & 2029 & N/A & N/A \\
\hline $2 \times 0.12 \mu \mathrm{m}$ PMOS & 803.6 & $1.33(11.08 \mathrm{x})$ & 2035 \\
\hline $3 \times 0.12 \mu \mathrm{m}$ PMOS & 502.7 & $2.45(20.42 \mathrm{x})$ & 2033 \\
\hline $1 \times 0.15 \mu \mathrm{m}$ NMOS & 1409 & N/A & N/A \\
\hline $2 \times 0.15 \mu \mathrm{m}$ NMOS & 542.6 & $0.65(4.33 \times)$ & 1425 \\
\hline $3 \times 0.15 \mu \mathrm{m}$ NMOS & 341.0 & $1.20(8.00 \mathrm{x})$ & 1415 \\
\hline
\end{tabular}


Table 16: $90 \mathrm{~nm}, V_{D D}=1.2 \mathrm{~V}$; stack currents and scaled widths

\begin{tabular}{|c|c|c|c|}
\hline Configuration & $\mathbf{I}[\boldsymbol{\mu A}]$ & Scaled W $[\boldsymbol{\mu m}]$ & Scaled $\mathbf{I}[\boldsymbol{\mu A}]$ \\
\hline $1 \times 0.41 \mu \mathrm{m}$ PMOS & 157.3 & N/A & N/A \\
\hline $2 \times 0.41 \mu \mathrm{m}$ PMOS & 75.86 & $0.85(2.07 \times)$ & 159.3 \\
\hline $3 \times 0.41 \mu \mathrm{m}$ PMOS & 50.25 & $1.21(2.95 \times)$ & 157.7 \\
\hline $1 \times 0.12 \mu \mathrm{m}$ NMOS & 111.8 & N/A & N/A \\
\hline $2 \times 0.12 \mu \mathrm{m}$ NMOS & 68.01 & $0.22(1.83 \times)$ & 115.3 \\
\hline $3 \times 0.12 \mu \mathrm{m}$ NMOS & 48.07 & $0.31(2.58 \mathrm{x})$ & 112.3 \\
\hline
\end{tabular}

Table 17: $90 \mathrm{~nm}, V_{D D}=0.3 \mathrm{~V}$; stack currents and scaled widths

\begin{tabular}{|c|c|c|c|}
\hline Configuration & I [nA] & Scaled W [ $\mu \mathrm{m}]$ & Scaled I [nA] \\
\hline $1 \times 0.41 \mu \mathrm{m}$ PMOS & 527.0 & N/A & N/A \\
\hline $2 \times 0.41 \mu \mathrm{m}$ PMOS & 222.6 & $0.90(2.20 \mathrm{x})$ & 532.9 \\
\hline $3 \times 0.41 \mu \mathrm{m}$ PMOS & 142.5 & $1.29(3.15 \mathrm{x})$ & 527.8 \\
\hline $1 \times 0.12 \mu \mathrm{m}$ NMOS & 811.0 & N/A & N/A \\
\hline $2 \times 0.12 \mu \mathrm{m}$ NMOS & 294.2 & $0.97(8.08 \mathrm{x})$ & 817.2 \\
\hline $3 \times 0.12 \mu \mathrm{m}$ NMOS & 180.1 & $1.52(12.67 \mathrm{x})$ & 812.1 \\
\hline
\end{tabular}

The subthreshold optimum widths found in Sections 4.1 and 4.2 were for a single transistor or an inverter configuration. If two or more transistors are required in series, from the subthreshold current of Equation (1), it stands to reason that while $V_{G S}$ and $V_{D S}$ will drop, the other parameters, mainly $V_{T}$, will remain largely unaffected. Thus while the absolute value of the current may decrease, it will retain the same behavior. From the circuit configurations shown in Figure 23, Figure 24 plots the current-to-capacitance ratio for the $65 \mathrm{~nm}$ LP transistors in subthreshold. The subthreshold optimum widths are 0.12 $\mu \mathrm{m}$ for both PMOS and NMOS, as was the case with a single transistor. 


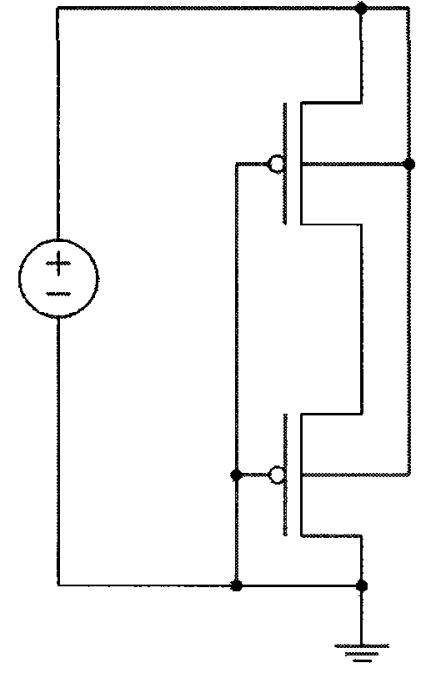

(a)

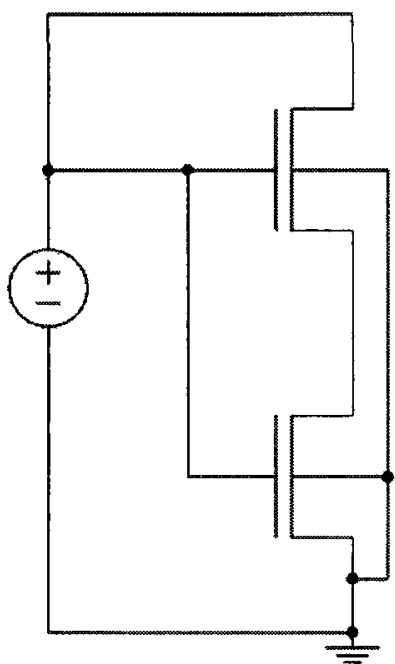

(b)

Figure 23: Double stack configurations for (a) PMOS and (b) NMOS

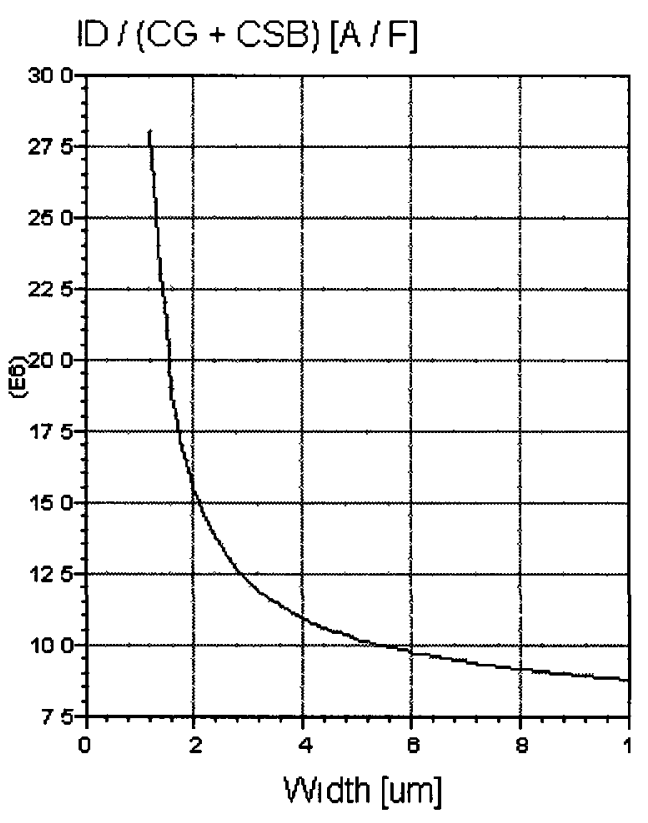

(a)

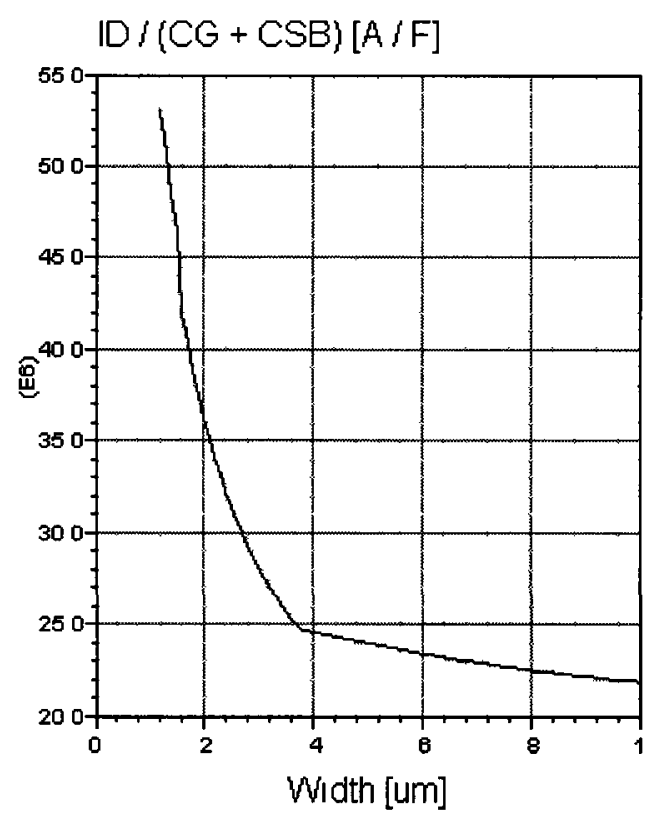

(b)

Figure 24: $65 \mathrm{~nm} \mathrm{LP,} V_{D D}=0.3 \mathrm{~V}$; current-to-capacitance ratio $\left(I_{D} /\left(C_{G}+C_{S B}\right)\right)$ versus width for two series (a) PMOS and (b) NMOS 
Likewise, Figure 25 confirms the $65 \mathrm{~nm}$ GP subthreshold optimum widths of 0.12 and $0.15 \mu \mathrm{m}$ for PMOS and NMOS, respectively. The subthreshold behavior for the 90 $\mathrm{nm}$ technology also is the same, as seen in Figure 26. The NMOS transistor has a subthreshold optimum width of $0.12 \mu \mathrm{m}$, while the PMOS transistor's current-tocapacitance ratio keeps increasing with the width. These results indicate that the same subthreshold optimum widths obtained when simulating a single transistor apply when considering multiple transistors in a stack.

$\mathrm{ID} /(\mathrm{CG}+\mathrm{CSB})[\mathrm{A} / \mathrm{F}]$

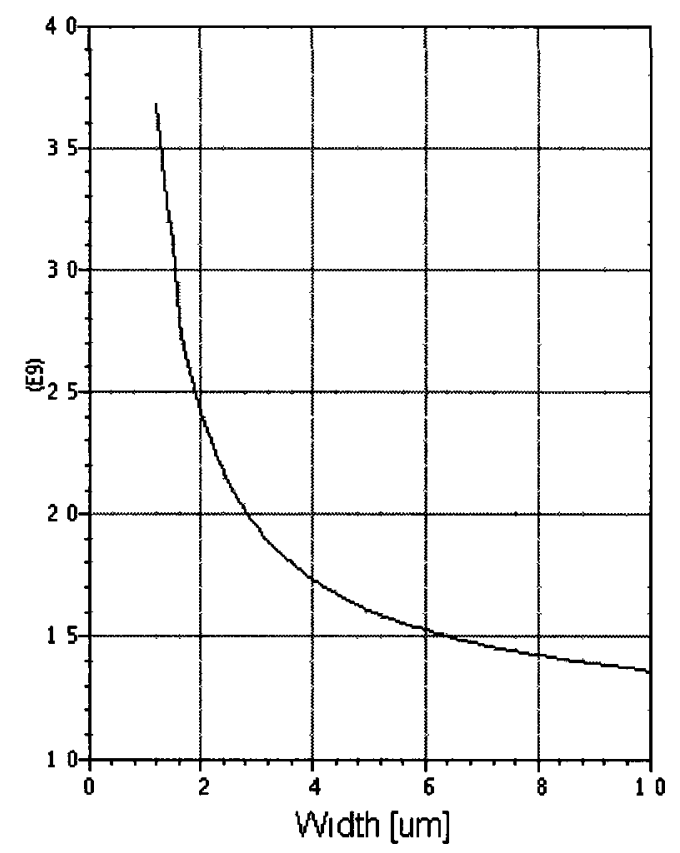

(a)
$\mathrm{ID} /(\mathrm{CG}+\mathrm{CSB})[\mathrm{A} / \mathrm{F}]$

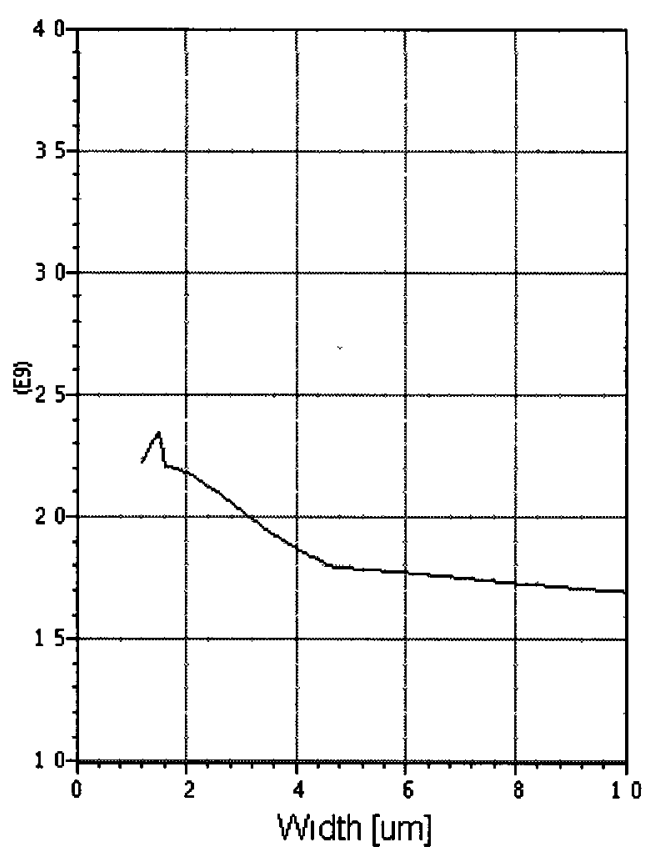

(b)

Figure 25: $65 \mathrm{~nm}$ GP, $V_{D D}=0.3 \mathrm{~V}$; current-to-capacitance ratio $\left(I_{D} /\left(C_{G}+C_{S B}\right)\right)$ versus width for two series (a) PMOS and (b) NMOS 


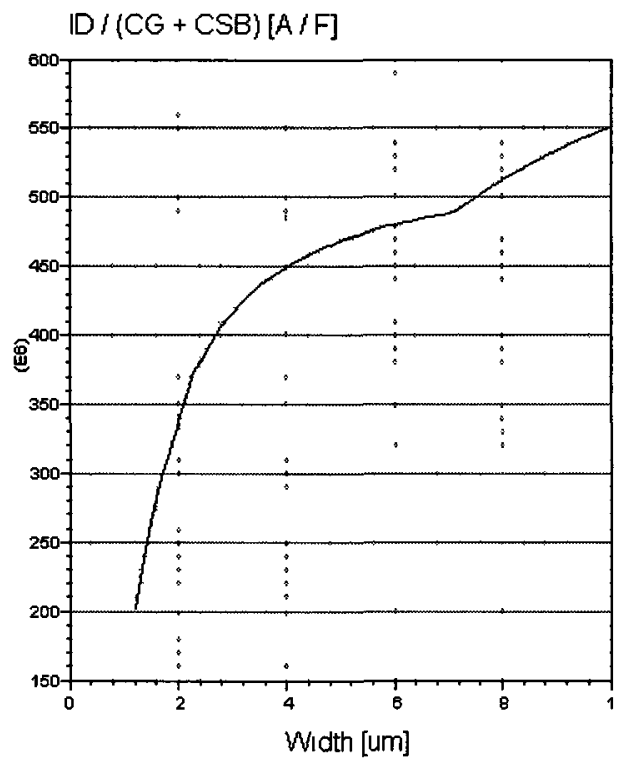

(a)

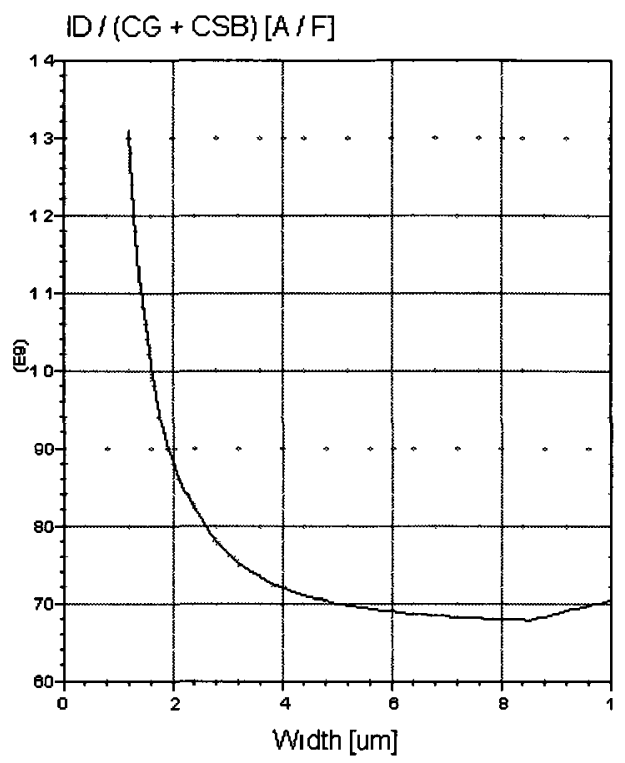

(b)

Figure 26: $90 \mathrm{~nm}, V_{D D}=0.3 \mathrm{~V}$; current-to-capacitance ratio $\left(I_{D} /\left(C_{G}+C_{S B}\right)\right)$ versus width for two series (a) PMOS and (b) NMOS

\subsection{Ring Oscillator Tests for Parallel Transistor Stacks}

As a test of the effectiveness of splitting a large transistor or a stack of large transistors into parallel transistor stacks, a 9-inverter ring oscillator will be simulated at $0.3 \mathrm{~V}$ in the $65 \mathrm{~nm}$ LP technology according to the two configurations shown in Figure 27. The standard inverter consists of large transistors with a beta ratio of 1.35 , the subthreshold effective beta ratio for this technology. The parallel inverter configuration splits the widths for the PMOS and NMOS transistors into parallel transistors sized at the subthreshold optimum width of $0.12 \mu \mathrm{m}$. Similarly, the two 3-input NAND gate configurations shown in Figure 28 were simulated in a 9-gate ring oscillator. Input $\mathrm{C}$ was used for the loop path while inputs $\mathrm{A}$ and $\mathrm{B}$ were tied to $V_{D D}$. 


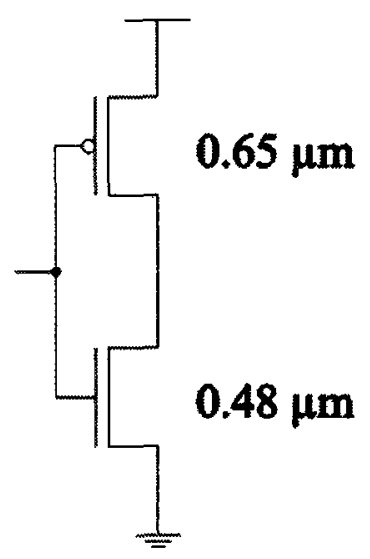

(a)

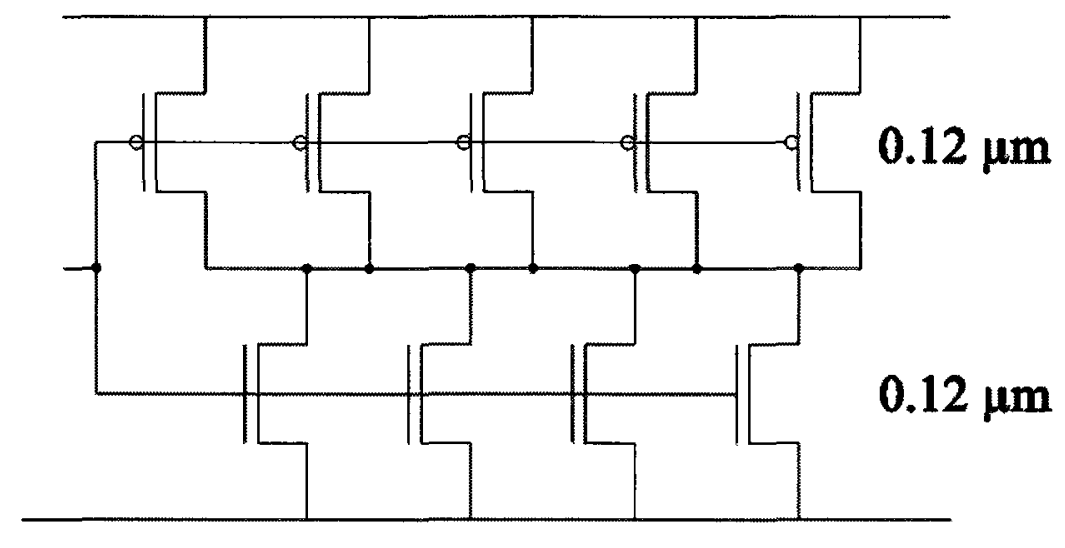

(b)

Figure 27: (a) Standard and (b) parallel inverter configurations for 9-inverter ring oscillator

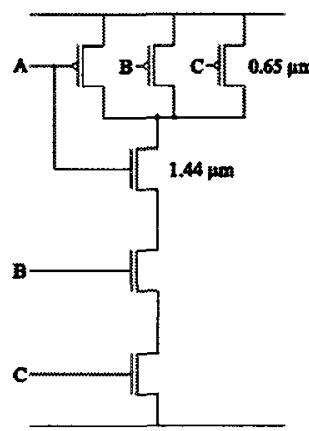

(a)

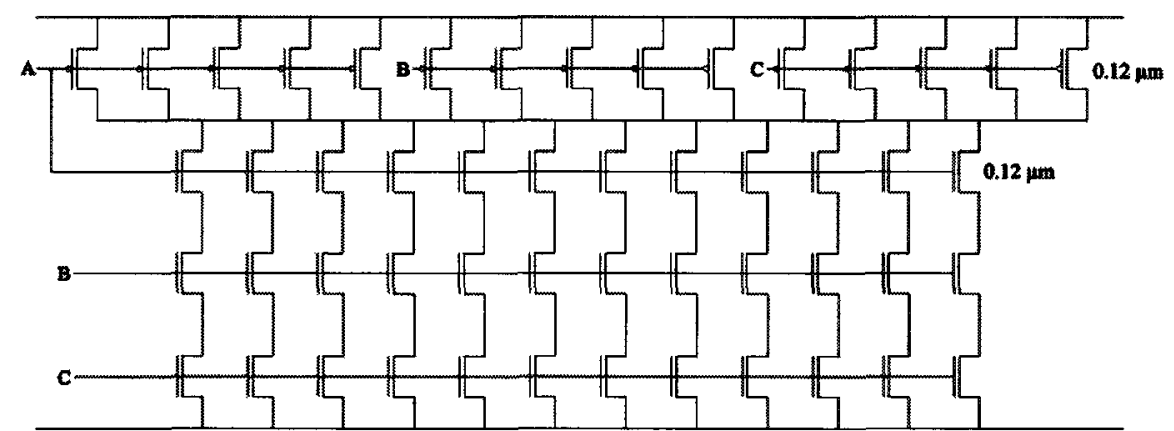

(b)

Figure 28: (a) Standard and (b) parallel 3-input NAND gate configurations for 9-gate ring oscillator

Table 18 contains the simulation results. The parallel configuration inverter is 2.63 times faster than its standard counterpart. The speed increase of the parallel 3-input NAND gate over its standard counterpart is 2.85 times. The reduction in energy-delay 
product is $49.2 \%$ and $56.2 \%$ for the inverter and 3-input NAND gate, respectively. This is approximately a doubling of the efficiency.

Table 18: $65 \mathrm{~nm} \mathrm{LP}, V_{D D}=0.3 \mathrm{~V}$; standard versus parallel configuration ring oscillator simulation results

\begin{tabular}{|l|c|c|c|c|c|}
\hline \multicolumn{1}{|c|}{ Gate } & $\mathbf{f}[\mathbf{M H z}]$ & $\mathbf{t}_{\mathrm{p}}[\mathbf{n s}]$ & $\mathbf{P}_{\text {avg }}[\mathbf{p W}]$ & $\mathbf{P D P}[\mathbf{a J}]$ & $\mathbf{E D P}[\mathbf{J} \cdot \mathbf{s}]$ \\
\hline INV - standard & 1.555 & 35.73 & 384.3 & 13.73 & $4.906 \mathrm{e}-25$ \\
\hline INV - parallel & 4.092 & 13.58 & 1351 & 18.35 & $2.491 \mathrm{e}-25$ \\
\hline NAND3 - standard & 0.4824 & 115.2 & 388.3 & 44.72 & $5.150 \mathrm{e}-24$ \\
\hline NAND3 - parallel & 1.374 & 40.43 & 1545 & 62.47 & $2.256 \mathrm{e}-24$ \\
\hline
\end{tabular}

\subsection{Subthreshold Logic Design Methodology}

All the steps of the developed design methodology from this chapter will now be numerated. For a given technology:

1. Determine the subthreshold optimum width for each transistor type. This can be done either through the current-to-capacitance ratio or from the ring oscillator simulations.

2. Find the parallel transistor ratio through ring oscillator simulations. Note that all transistors should be sized at their subthreshold optimum width.

3. Calculate the effective beta ratio by multiplying the parallel transistor ratio with the ratio of the PMOS-to-NMOS subthreshold optimum widths.

4. Design the circuit with the sizing strategy of choice such as Logical Effort using the effective beta ratio. 
5. Convert the transistor sizes into number of parallel transistors by dividing the designed size by the subthreshold optimum width and rounding to the nearest integer.

6. Create the circuit using parallel transistor stacks. The sizes of all transistors are fixed to the subthreshold optimum width including those of stacked transistors. The number of transistors in parallel was determined in step 5 .

As an example using Logical Effort, suppose a NAND gate in the $90 \mathrm{~nm}$ technology kit is being designed. The effective beta ratio is 2.56 , thus a normalized unit inverter will have PMOS and NMOS widths of 2.56 and 1, respectively. Assuming a doubling of the width for two series transistors, both of a unit NAND gate's PMOS transistors will have widths of 2.56 , while the two NMOS transistors will have widths of 2. Now suppose that Logical Effort has resulted in a total width of $2 \mu \mathrm{m}$ for a given input of the NAND gate. This will be divided as $1.12 \mu \mathrm{m}$ for PMOS and $0.88 \mu \mathrm{m}$ for the NMOS. The subthreshold optimum width for the PMOS transistor is $0.41 \mu \mathrm{m}$, thus 1.12 $\mu \mathrm{m}$ divided by $0.41 \mu \mathrm{m}$, rounded to the nearest integer, results in 3 parallel stacks of PMOS transistors. Similarly, given the subthreshold optimum width of $0.12 \mu \mathrm{m}$ for the NMOS transistor, $0.88 \mu \mathrm{m}$ divided by $0.12 \mu \mathrm{m}$ results in 7 parallel stacks. The division of the gate into parallel transistors is shown in Figure 29. 

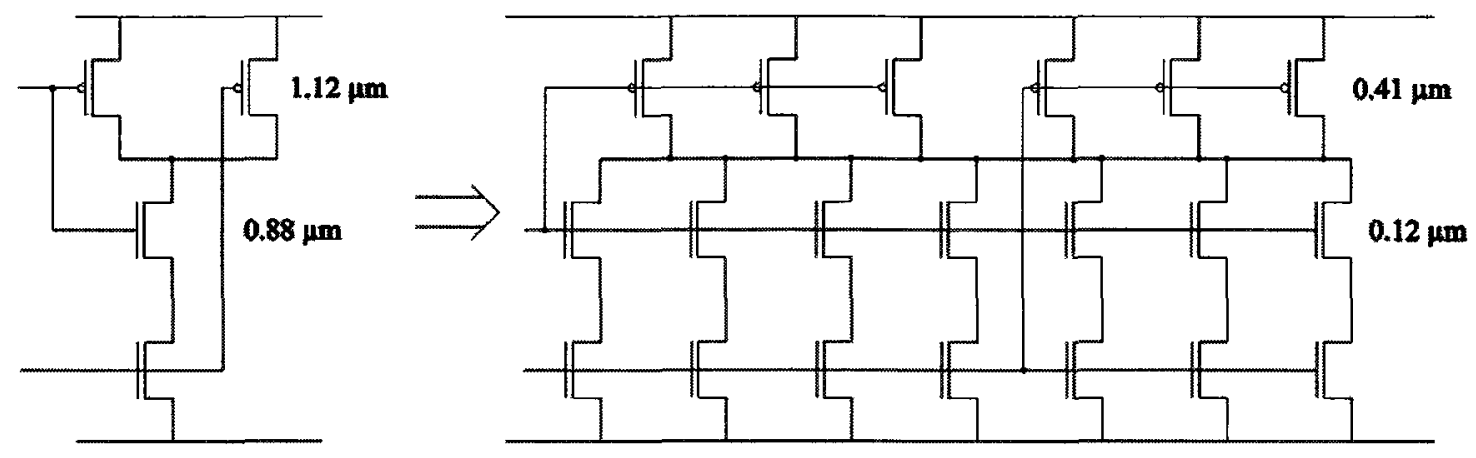

Figure 29: NAND split into parallel transistors 


\section{CHAPTER 5 - APPLICATION CIRCUITS}

The design methodology developed in the preceding chapter will be put to the test in two application circuits. Comparisons with a standard design methodology are provided. The first test circuit is a chain of five gates with two different loads. The second circuit is a 32-bit carry-lookahead adder where both schematic and post-layout simulations are performed.

\subsection{Test Circuit}

The propagation delays of a custom test circuit composed of five logic gates have been measured. The two propagation delays $t_{p H L}\left(\mathrm{~V}_{\mathrm{in}}\right.$ rising, $\mathrm{V}_{\text {out }}$ falling $)$ and $t_{p L H}\left(\mathrm{~V}_{\mathrm{in}}\right.$ falling, $V_{\text {out }}$ rising) are measured from $V_{1 n}$ to $V_{\text {out }}$, as shown in Figure 30 . The propagation delay $t_{p}$ is the average of $t_{p H L}$ and $t_{p L H}$. The circuit consists of the following gates: INV $\rightarrow$ NAND2 $\rightarrow$ NOR2 $\rightarrow$ NOR3 $\rightarrow$ NAND3. The chain has been constructed for worst case performance such that the input excited in the chain path is that furthest from the output of a given gate. In order to allow the circuit to function, the unused inputs of the NAND gates are tied to $V_{D D}$, while those of the NOR gates are tied to GND. The order of the gates was chosen in order to avoid having both either the pull-up or pulldown networks of the NAND or NOR gates active during one simulation. For example, should $V_{1 n}$ be rising, the pull-up network of the NAND2 and the pull-down network of the NAND3 will be active. This is to help balance any performance discrepancy should any gate type be better at conducting in its pull-up or pull-down networks. 


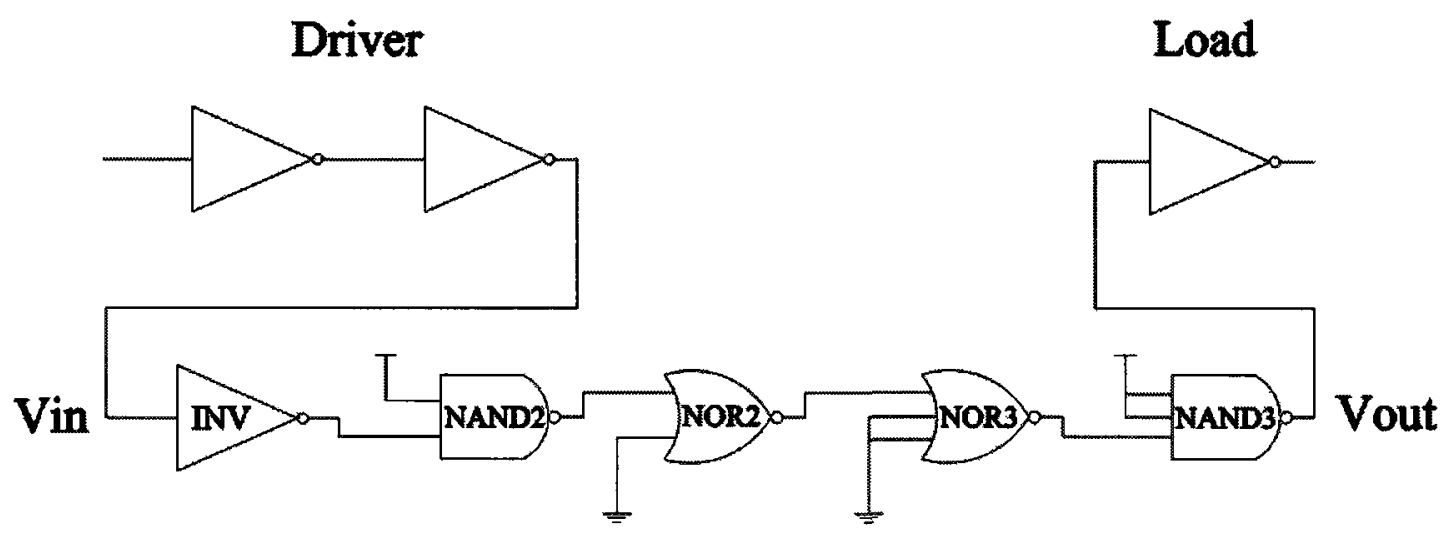

Figure 30: Test circuit schematic

$V_{1 n}$ is generated through a driver circuit consisting of two inverters. The circuit has been designed to ensure that the rise and fall times of $V_{\mathrm{In}}$ are well within a $10 \%$ difference of each other.

Two loading conditions have been tested: a single inverter composed of PMOS and NMOS widths of 0.24 and $0.12 \mu \mathrm{m}$, respectively, and 32 of these inverters in parallel. The application of Logical Effort for the gate sizing in the case of a single inverter load may have resulted in transistor widths below the minimum of $0.12 \mu \mathrm{m}$. In these cases, the minimum width was used.

Four beta ratio values have been chosen for the Logical Effort sizing. A value of 2 is being used since this is a typical value used in textbooks when teaching Logical Effort that approximates a symmetric inverter response. The second beta ratio chosen was the value that maximized the superthreshold ring oscillator frequency. From Tables 3, 6 and 8 , it can be seen that the ratio varied depending upon the NMOS width. As such, 
the ratio value of the highest frequency was used as a representative value for the technology. Third, a beta ratio calculated from the ratio of the PMOS-to-NMOS subthreshold optimum widths. These simulations will show the effect of neglecting the parallel transistor ratio. Finally, the effective beta ratios of Table 11 which were found for the subthreshold design methodology were simulated. For each beta ratio, two configurations were tested. The first is a standard configuration where the resulting widths from Logical Effort were simulated as a single transistor. The second configuration, parallel, splits this width into parallel transistors all sized at the subthreshold optimum width. Since only an integer number of transistors can be used, the total width of the transistors may be different from the calculated width. This is partially the reason that the sum of the widths of the transistors of the five gates in the chain are provided. Note that this width is for one input only. For example, a standard NAND3 gate with $0.24 \mu \mathrm{m}$ PMOS and $0.36 \mu \mathrm{m}$ NMOS transistors would contribute $0.6 \mu \mathrm{m}$ to the total width, not the $1.8 \mu \mathrm{m}$ for all the transistors of all three inputs.

One important note is how the logical effort values of the gates have been determined. As is typical, the logical effort of all gates is normalized to that of the unit inverter, thus the inverter, regardless of the sizing of the PMOS and NMOS transistors, has a logical effort of 1 . If considering the NAND2 gate, the two series NMOS transistors would be scaled to provide the same current drive as the NMOS transistor of the inverter. However, since the beta ratios and thus inverter sizes have been determined from both superthreshold and subthreshold operation, the question becomes which operating voltage to use to determine the required scaling. In order to keep the focus on 
comparing the beta ratios only and for simplicity, a convention of doubling the width of two and tripling the width of three series transistors will be used.

Also simulated are the propagation delays of a chain of gates composed of minimum sized transistors.

Before examining the simulation results, the design for the $65 \mathrm{~nm}$ GP parallel configuration with subthreshold effective beta ratio under the large load conditions will be detailed. This will demonstrate the proper application of Logical Effort with the subthreshold design methodology. The first step is to find the subthreshold optimum widths for both transistors. From the prior simulations and Table 11, we know that these widths are $0.12 \mu \mathrm{m}$ for PMOS and $0.15 \mu \mathrm{m}$ for NMOS. The parallel transistor ratio is 0.95. Thus the effective beta ratio is 0.76 . Next, we apply Logical Effort to the test circuit shown in Figure 30.

The calculations are summarized in Table 19. A normalized unit width will be equivalent to $0.12 \mu \mathrm{m}$. Thus INV, which will be considered a unit inverter, will have PMOS and NMOS widths of 1 and 1.32, respectively. By definition, the logical effort of this first gate is 1 . After finding the sizes of the other unit gates, their logical efforts can be calculated. The path logical effort is found by multiplying all the gate logical efforts together. The path input, i.e., the INV gate, has a total width of 2.32 . The path output, or load, has a total width of 3 for one inverter multiplied by 32 inverters in parallel, thus 96 . The path electrical effort can then be calculated as $96 / 2.32=41.4$. The path effort is then found by multiplying the path logical effort with the path electrical effort. Next, the best stage effort is calculated. With this, the total widths per input for the five gates are 
found. This is then split between the PMOS and NMOS transistors, and then converted into microns. Now, the widths are divided by the subthreshold optimum widths to calculate the required number of transistors in parallel.

Table 19: $65 \mathrm{~nm}$ GP; parallel configuration with effective beta ratio under large load design example

\begin{tabular}{|c|c|c|c|c|c|}
\hline & INV & NAND2 & NOR2 & NOR3 & NAND3 \\
\hline $\begin{array}{l}\text { Normalized unit gate } \\
\text { PMOS / NMOS width }\end{array}$ & $1 / 1.32$ & $1 / 2.64$ & $2 / 1.32$ & $3 / 1.32$ & $1 / 3.96$ \\
\hline Logical effort & 1 & 1.57 & 1.43 & 1.86 & 2.14 \\
\hline Path logical effort & \multicolumn{5}{|c|}{8.94} \\
\hline Path electrical effort & \multicolumn{5}{|c|}{$(32 \times 3) /(1+1.32)=41.4$} \\
\hline Path effort & \multicolumn{5}{|c|}{$8.94 \times 41.4=370$} \\
\hline Best stage effort & \multicolumn{5}{|c|}{$370^{1 / 5}=3.26$} \\
\hline $\begin{array}{l}\text { Total normalized } \\
\text { width per input }\end{array}$ & 2.32 & 7.57 & 15.7 & 35.9 & 62.9 \\
\hline $\begin{array}{l}\text { Normalized } \\
\text { PMOS / NMOS width }\end{array}$ & $1 / 1.32$ & $2.08 / 5.49$ & $9.46 / 6.24$ & $24.9 / 11.0$ & $12.7 / 50.2$ \\
\hline $\begin{array}{l}\text { PMOS / NMOS width } \\
{[\mu \mathrm{m}]}\end{array}$ & $0.12 / 0.16$ & $0.25 / 0.66$ & $1.14 / 0.75$ & $2.99 / 1.32$ & $1.52 / 6.03$ \\
\hline $\begin{array}{l}\text { \# of PMOS / NMOS } \\
\text { transistors in parallel }\end{array}$ & $1 / 1$ & $2 / 4$ & $10 / 5$ & $25 / 9$ & $13 / 40$ \\
\hline
\end{tabular}

Table 20 contains the simulation results for the $65 \mathrm{~nm} \mathrm{LP}$ technology at $1.2 \mathrm{~V}$. For the small load, a beta ratio of 2 using a standard configuration was best, although the difference to the standard configuration with a beta ratio of 1.49 (superthreshold beta ratio) was only $0.9 \%$. The parallel configurations were only slightly slower than their standard counterparts. The performance of the minimum sized gates was in line with those of the other configurations. 
For the large load, the minimum sized gates were much slower. The 1.49 beta ratio standard configuration was best, although the difference to the other beta ratios was slight. The parallel configurations again lagged behind their standard counterparts.

Table 20: $65 \mathrm{~nm} L P, V_{D D}=1.2 \mathrm{~V}$; test circuit propagation delays

\begin{tabular}{|c|c|c|c|c|c|}
\hline $\boldsymbol{\beta}$ & Configuration & Width [um] & $\mathbf{t}_{\mathrm{pHL}}$ [ps] & $\mathbf{t}_{\mathrm{pLH}}$ [ps] & $t_{p}[p s]$ \\
\hline \multicolumn{6}{|c|}{ Small load - 1 inverter } \\
\hline \multirow[t]{2}{*}{2} & Standard & 2.58 & 146.2 & 134.5 & 140.4 \\
\hline & Parallel & 2.4 & 149.5 & 148.0 & 148.8 \\
\hline \multirow[t]{2}{*}{1.49} & Standard & 2.3 & 143.9 & 139.3 & 141.6 \\
\hline & Parallel & 2.28 & 142.4 & 150.8 & 146.6 \\
\hline \multirow[t]{2}{*}{1} & Standard & 2.04 & 146.6 & 144.8 & 145.7 \\
\hline & Parallel & 2.04 & 157.7 & 155.9 & 156.8 \\
\hline \multirow[t]{2}{*}{1.35} & Standard & 2.21 & 144.2 & 140.6 & 142.4 \\
\hline & Parallel & 2.16 & 139.1 & 160.7 & 149.9 \\
\hline \multicolumn{2}{|c|}{ Min. size transistors $(0.12 \mu \mathrm{m})$} & 1.2 & 158.2 & 128.6 & 143.4 \\
\hline \multicolumn{6}{|c|}{ Large load -32 inverters } \\
\hline \multirow[t]{2}{*}{2} & Standard & 14.98 & 174.4 & 184.8 & 179.6 \\
\hline & Parallel & 15.12 & 201.0 & 204.7 & 202.9 \\
\hline \multirow[t]{2}{*}{1.49} & Standard & 14.35 & 172.0 & 185.1 & 178.6 \\
\hline & Parallel & 14.52 & 200.7 & 195.7 & 198.2 \\
\hline \multirow[t]{2}{*}{1} & Standard & 13.77 & 176.2 & 197.0 & 186.6 \\
\hline & Parallel & 13.8 & 207.0 & 214.8 & 210.9 \\
\hline \multirow[t]{2}{*}{1.35} & Standard & 14.18 & 172.0 & 187.4 & 179.7 \\
\hline & Parallel & 14.28 & 194.4 & 217.9 & 206.2 \\
\hline \multicolumn{2}{|c|}{ Min. size transistors $(0.12 \mu \mathrm{m})$} & 1.2 & 530.4 & 405.2 & 467.8 \\
\hline
\end{tabular}

Table 21 contains the subthreshold results. Here, the parallel configurations completely dominate their standard counterparts. For the large load, the propagation delays for the standard configurations were more than double those of the parallel configurations. A beta ratio of 1.49 proved best for both loads, although the differences 
to the other beta ratios were small. The performance of the minimum sized gates was quite good for the small load. This shouldn't be too surprising since in the $65 \mathrm{~nm}$ LP technology, the minimum width is the same as the subthreshold optimum.

For the 1.35 parallel configurations, designed according to the methodology of this thesis, the reduction in delay compared to the fastest standard configurations were $41.0 \%$ and $61.4 \%$ for the small and large loads, respectively.

Table 21: $65 \mathrm{~nm} \mathrm{LP}, V_{D D}=0.3 \mathrm{~V}$; test circuit propagation delays

\begin{tabular}{|c|c|c|c|c|c|}
\hline$\beta$ & Configuration & Width [ $\mu \mathrm{m}]$ & $t_{\mathrm{pHL}}[\mathrm{ns}]$ & $\mathbf{t}_{\mathrm{pLH}}[\mathrm{ns}]$ & $\mathbf{t}_{\mathrm{p}}[\mathrm{ns}]$ \\
\hline \multicolumn{6}{|c|}{ Small load -1 inverter } \\
\hline \multirow[t]{2}{*}{2} & Standard & 2.58 & 297.7 & 231.3 & 264.5 \\
\hline & Parallel & 2.4 & 150.5 & 133.6 & 142.1 \\
\hline \multirow[t]{2}{*}{1.49} & Standard & 2.3 & 291.3 & 218.8 & 255.1 \\
\hline & Parallel & 2.28 & 144.9 & 136.0 & 140.5 \\
\hline \multirow[t]{2}{*}{1} & Standard & 2.04 & 283.0 & 202.4 & 242.7 \\
\hline & Parallel & 2.04 & 160.3 & 137.5 & 148.9 \\
\hline \multirow[t]{2}{*}{1.35} & Standard & 2.21 & 289.0 & 214.5 & 251.8 \\
\hline & Parallel & 2.16 & 141.3 & 145.1 & 143.2 \\
\hline \multicolumn{2}{|c|}{ Min. size transistors $(0.12 \mu \mathrm{m})$} & 1.2 & 174.0 & 129.8 & 151.9 \\
\hline \multicolumn{6}{|c|}{ Large load -32 inverters } \\
\hline \multirow[t]{2}{*}{2} & Standard & 14.98 & 535.6 & 491.3 & 513.5 \\
\hline & Parallel & 15.12 & 201.8 & 186.6 & 194.2 \\
\hline \multirow[t]{2}{*}{1.49} & Standard & 14.35 & 536.6 & 489.3 & 513.0 \\
\hline & Parallel & 14.52 & 202.9 & 177.9 & 190.4 \\
\hline \multirow[t]{2}{*}{1} & Standard & 13.77 & 549.6 & 502.5 & 526.1 \\
\hline & Parallel & 13.8 & 212.2 & 194.5 & 203.4 \\
\hline \multirow[t]{2}{*}{1.35} & Standard & 14.18 & 538.4 & 491.2 & 514.8 \\
\hline & Parallel & 14.28 & 197.4 & 198.2 & 197.8 \\
\hline \multicolumn{2}{|c|}{ Min. size transistors $(0.12 \mu \mathrm{m})$} & 1.2 & 465.7 & 293.6 & 379.7 \\
\hline
\end{tabular}


The $65 \mathrm{~nm}$ GP superthreshold results are shown in Table 22. Many of the same conclusions from the $65 \mathrm{~nm}$ LP superthreshold results still hold. A beta ratio of 2 proved best for the small load, while beta ratios of 2 and 1.31 proved almost equal for the large load.

Table 22: $65 \mathrm{~nm}$ GP, $V_{D D}=1 \mathrm{~V}$; test circuit propagation delays

\begin{tabular}{|c|c|c|c|c|c|}
\hline$\beta$ & Configuration & Width [ $\mu \mathrm{m}]$ & $\mathbf{t}_{\mathrm{pHL}}$ [ps] & $\mathbf{t}_{\mathrm{pLH}}[\mathrm{ps}]$ & $t_{p}[p s]$ \\
\hline \multicolumn{6}{|c|}{ Small load -1 inverter } \\
\hline \multirow[t]{2}{*}{2} & Standard & 2.58 & 80.41 & 74.67 & 77.54 \\
\hline & Parallel & 2.61 & 79.28 & 81.57 & 80.43 \\
\hline \multirow[t]{2}{*}{1.31} & Standard & 2.21 & 78.57 & 78.80 & 78.69 \\
\hline & Parallel & 2.37 & 74.14 & 89.58 & 81.86 \\
\hline \multirow[t]{2}{*}{0.8} & Standard & 2.22 & 81.05 & 82.58 & 81.82 \\
\hline & Parallel & 2.01 & 81.28 & 84.22 & 82.75 \\
\hline \multirow[t]{2}{*}{0.76} & Standard & 2.23 & 82.13 & 83.57 & 82.85 \\
\hline & Parallel & 2.16 & 81.53 & 93.04 & 87.29 \\
\hline \multicolumn{2}{|c|}{ Min. size transistors $(0.12 \mu \mathrm{m})$} & 1.2 & 88.66 & 71.20 & 79.93 \\
\hline \multicolumn{6}{|c|}{ Large load -32 inverters } \\
\hline \multirow[t]{2}{*}{2} & Standard & 14.98 & 100.9 & 105.6 & 103.3 \\
\hline & Parallel & 15.18 & 106.0 & 111.2 & 108.6 \\
\hline \multirow[t]{2}{*}{1.31} & Standard & 14.13 & 99.61 & 106.9 & 103.3 \\
\hline & Parallel & 14.34 & 99.99 & 118.3 & 109.1 \\
\hline \multirow[t]{2}{*}{0.8} & Standard & 14.69 & 104.1 & 117.1 & 110.6 \\
\hline & Parallel & 14.67 & 105.9 & 121.4 & 113.7 \\
\hline \multirow[t]{2}{*}{0.76} & Standard & 14.92 & 104.8 & 119.0 & 111.9 \\
\hline & Parallel & 14.97 & 106.8 & 120.3 & 113.6 \\
\hline \multicolumn{2}{|c|}{ Min. size transistors $(0.12 \mu \mathrm{m})$} & 1.2 & 310.5 & 217.5 & 264.0 \\
\hline
\end{tabular}


The subthreshold results for $65 \mathrm{~nm}$ GP are in Table 23. The gains from the parallel configurations compared to the standard configurations are not as great as in the $65 \mathrm{~nm}$ LP technology, however, they are still significant. A beta ratio of 0.8 was best for the small load, while a beta ratio of 0.76 proved best for the large load.

For the 0.76 parallel configurations, designed according to the methodology of this thesis, the reduction in delay compared to the fastest standard configurations were $19.8 \%$ and $45.3 \%$ for the small and large loads, respectively.

Table 23: $65 \mathrm{~nm} \mathrm{GP}, V_{D D}=0.3 \mathrm{~V}$; test circuit propagation delays

\begin{tabular}{|c|c|c|c|c|c|}
\hline $\boldsymbol{\beta}$ & Configuration & Width $[\mu \mathrm{m}]$ & $\mathbf{t}_{\mathrm{pHL}}[\mathrm{ns}]$ & $t_{\mathrm{pLH}}[\mathrm{ns}]$ & $t_{p}[n s]$ \\
\hline \multicolumn{6}{|c|}{ Small load -1 inverter } \\
\hline \multirow[t]{2}{*}{2} & Standard & 2.58 & 3.022 & 2.381 & 2.702 \\
\hline & Parallel & 2.61 & 2.143 & 1.898 & 2.021 \\
\hline \multirow[t]{2}{*}{1.31} & Standard & 2.21 & 2.774 & 2.193 & 2.484 \\
\hline & Parallel & 2.37 & 1.984 & 1.973 & 1.979 \\
\hline \multirow[t]{2}{*}{0.8} & Standard & 2.22 & 2.696 & 2.058 & 2.377 \\
\hline & Parallel & 2.01 & 1.977 & 1.752 & 1.865 \\
\hline \multirow[t]{2}{*}{0.76} & Standard & 2.23 & 2.680 & 2.063 & 2.372 \\
\hline & Parallel & 2.16 & 1.947 & 1.857 & 1.902 \\
\hline \multicolumn{2}{|c|}{ Min. size transistors $(0.12 \mu \mathrm{m})$} & 1.2 & 2.239 & 1.640 & 1.940 \\
\hline \multicolumn{6}{|c|}{ Large load -32 inverters } \\
\hline \multirow[t]{2}{*}{2} & Standard & 14.98 & 5.108 & 4.601 & 4.855 \\
\hline & Parallel & 15.18 & 3.081 & 2.809 & 2.945 \\
\hline \multirow[t]{2}{*}{1.31} & Standard & 14.13 & 4.803 & 4.306 & 4.555 \\
\hline & Parallel & 14.34 & 2.602 & 2.636 & 2.619 \\
\hline \multirow[t]{2}{*}{0.8} & Standard & 14.69 & 4.713 & 4.293 & 4.503 \\
\hline & Parallel & 14.67 & 2.512 & 2.449 & 2.481 \\
\hline \multirow[t]{2}{*}{0.76} & Standard & 14.92 & 4.733 & 4.323 & 4.528 \\
\hline & Parallel & 14.97 & 2.531 & 2.394 & 2.463 \\
\hline \multicolumn{2}{|c|}{ Min. size transistors $(0.12 \mu \mathrm{m})$} & 1.2 & 12.64 & 2.729 & 7.685 \\
\hline
\end{tabular}


Table 24 contains the $1.2 \mathrm{~V}$ simulations for the $90 \mathrm{~nm}$ technology. For the small load, three of the parallel configurations resulted in the same circuit considering the wide subthreshold optimum width of the PMOS transistor. Ultimately, these parallel configurations proved marginally better than the standard configurations.

For the large load, the standard configurations for beta ratios of 2 and 1.58 proved best, while all the standard configurations were reliably faster than the parallel configurations.

Table 24: $90 \mathrm{~nm}, V_{D D}=1.2 \mathrm{~V}$; test circuit propagation delays

\begin{tabular}{|c|c|c|c|c|c|}
\hline$\beta$ & Configuration & Width [ $\mu \mathrm{m}]$ & $t_{\mathrm{pHL}}[\mathrm{ps}]$ & $t_{\mathrm{pLH}}[\mathrm{ps}]$ & $t_{p}$ [ps] \\
\hline \multicolumn{6}{|c|}{ Small load -1 inverter } \\
\hline \multirow{2}{*}{2} & Standard & 2.58 & 107.0 & 107.5 & 107.3 \\
\hline & Parallel & 2.89 & 114.8 & 95.13 & 105.0 \\
\hline \multirow[t]{2}{*}{1.58} & Standard & 2.36 & 106.4 & 113.2 & 109.8 \\
\hline & Parallel & 2.89 & 114.8 & 95.13 & 105.0 \\
\hline \multirow[t]{2}{*}{3.42} & Standard & 3.24 & 113.7 & 102.5 & 108.1 \\
\hline & Parallel & 3.59 & 132.4 & 104.4 & 118.4 \\
\hline \multirow[t]{2}{*}{2.56} & Standard & 2.85 & 110.1 & 103.8 & 107.0 \\
\hline & Parallel & 2.89 & 114.8 & 95.13 & 105.0 \\
\hline \multicolumn{2}{|c|}{ Min. size transistors $(0.12 \mu \mathrm{m})$} & 1.2 & 131.9 & 112.7 & 122.3 \\
\hline \multicolumn{6}{|c|}{ Large load - 32 inverters } \\
\hline \multirow[t]{2}{*}{2} & Standard & 14.98 & 119.6 & 138.0 & 128.8 \\
\hline & Parallel & 15.07 & 150.3 & 145.5 & 147.9 \\
\hline \multirow[t]{2}{*}{1.58} & Standard & 14.45 & 118.3 & 139.3 & 128.8 \\
\hline & Parallel & 14.56 & 141.8 & 151.4 & 146.6 \\
\hline \multirow[t]{2}{*}{3.42} & Standard & 16.79 & 129.4 & 151.6 & 140.5 \\
\hline & Parallel & 16.74 & 146.0 & 163.7 & 154.9 \\
\hline \multirow[t]{2}{*}{2.56} & Standard & 15.71 & 122.8 & 141.6 & 132.2 \\
\hline & Parallel & 16.11 & 140.6 & 153.4 & 147.0 \\
\hline \multicolumn{2}{|c|}{ Min. size transistors $(0.12 \mu \mathrm{m})$} & 1.2 & 332.5 & 416.1 & 374.3 \\
\hline
\end{tabular}


In Table 25, the subthreshold $90 \mathrm{~nm}$ results are shown. In all cases, the parallel configurations proved faster than their standard counterparts, although not always by the greatest of margins. As mentioned previously, for the small load, three of the parallel configurations resulted in the same circuit. For the large load, a beta ratio of 2.56 proved best.

For the 2.56 parallel configurations, designed according to the methodology of this thesis, the reduction in delay compared to the fastest standard configurations were $7.4 \%$ and $16.0 \%$ for the small and large loads, respectively.

Table 25: $90 \mathrm{~nm}, V_{D D}=0.3 \mathrm{~V}$; test circuit propagation delays

\begin{tabular}{|c|c|c|c|c|c|}
\hline$\beta$ & Configuration & 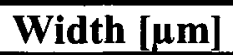 & $t_{\mathrm{pHL}}[\mathrm{ns}]$ & $t_{\mathrm{pLH}}[\mathrm{ns}]$ & $t_{p}[n s]$ \\
\hline \multicolumn{6}{|c|}{ Small load -1 inverter } \\
\hline \multirow[t]{2}{*}{2} & Standard & 2.58 & 9.847 & 9.511 & 9.679 \\
\hline & Parallel & 2.89 & 9.530 & 7.221 & 8.376 \\
\hline \multirow[t]{2}{*}{1.58} & Standard & 2.36 & 10.40 & 10.47 & 10.44 \\
\hline & Parallel & 2.89 & 9.530 & 7.221 & 8.376 \\
\hline \multirow[t]{2}{*}{3.42} & Standard & 3.24 & 9.312 & 8.788 & 9.050 \\
\hline & Parallel & 3.59 & 9.951 & 7.313 & 8.632 \\
\hline \multirow[t]{2}{*}{2.56} & Standard & 2.85 & 9.597 & 8.970 & 9.284 \\
\hline & Parallel & 2.89 & 9.530 & 7.221 & 8.376 \\
\hline \multicolumn{2}{|c|}{ Min. size transistors $(0.12 \mu \mathrm{m})$} & 1.2 & 18.71 & 14.43 & 16.57 \\
\hline \multicolumn{6}{|c|}{ Large load - 32 inverters } \\
\hline \multirow[t]{2}{*}{2} & Standard & 14.98 & 11.87 & 13.39 & 12.63 \\
\hline & Parallel & 15.07 & 12.03 & 10.13 & 11.08 \\
\hline \multirow[t]{2}{*}{1.58} & Standard & 14.45 & 11.77 & 13.51 & 12.64 \\
\hline & Parallel & 14.56 & 11.78 & 10.87 & 11.33 \\
\hline \multirow[t]{2}{*}{3.42} & Standard & 16.79 & 12.89 & 14.79 & 13.84 \\
\hline & Parallel & 16.74 & 10.97 & 10.63 & 10.80 \\
\hline \multirow[t]{2}{*}{2.56} & Standard & 15.71 & 12.20 & 13.80 & 13.00 \\
\hline & Parallel & 16.11 & 10.79 & 10.42 & 10.61 \\
\hline \multicolumn{2}{|c|}{ Min. size transistors $(0.12 \mu \mathrm{m})$} & 1.2 & 30.40 & 48.52 & 39.46 \\
\hline
\end{tabular}


For all three technologies, there was a benefit to using parallel stacks of transistors of the subthreshold optimum width. In the $65 \mathrm{~nm}$ LP and GP technologies, the increase in performance when using parallel stacks instead of the standard configuration was greater for the larger load. This can be attributed to the fact that the standard gates in the large load scenario have transistors whose widths are much larger than the subthreshold optimum width. That is one reason why the gains between the small and large loads in the $90 \mathrm{~nm}$ technology are comparable. Also, the $90 \mathrm{~nm}$ PMOS transistor exhibits narrow-width effect and thus does not benefit from being narrow in subthreshold.

The effective beta ratios for subthreshold design also proved best in two of the three technologies. Although it did not give the best results in $65 \mathrm{~nm} \mathrm{LP}$, the effective beta ratio of 1.35 performed reasonably when compared to the superthreshold optimum beta ratio of 1.49 . These variations can be expected since there is a broad minimum for the delay with respect to the beta ratio.

\subsection{Carry-Lookahead Adder}

A 32-bit carry-lookahead adder produces a sum $S[32: 1]$ and carry-out $\mathrm{C}_{\text {out }}$ from inputs $A[32: 1], B[32: 1]$ and carry-in $C_{10}$. The adder being designed will be made from eight valency-4 blocks. One block is shown in Figure 31. Bitwise propagate and generate (PG) signals are evaluated for the four bits of A and B. Propagate and generate refer to the carry; the carry-in may be propagated to or a carry-out generated for the next part of the circuit. The bitwise PG signals are then combined with $C_{\text {nn }}$ to produce four 
output bits of $S . C_{n}$ refers to either the $C_{1 n}$ input to the adder if this is the first block or is otherwise the signal $\mathrm{C}_{\text {out }}$ from a previous block. The bitwise PG signals are also used to generate group PG signals. The group PG signals are then combined with $C_{1 n}$ in the carry logic to determine if there is a $C_{\text {out }}$. The $C_{\text {out }}$ of the block then becomes the $C_{n}$ of the next block, or is the $\mathrm{C}_{\text {out }}$ of the adder if considering the last block. By evaluating the carry-out of a 4-bit group in parallel with the sum bits and passing it on to the next block, the addition process is sped up compared to a carry-ripple adder where each sum bit must wait on receiving the carry from the previous full adder [13].

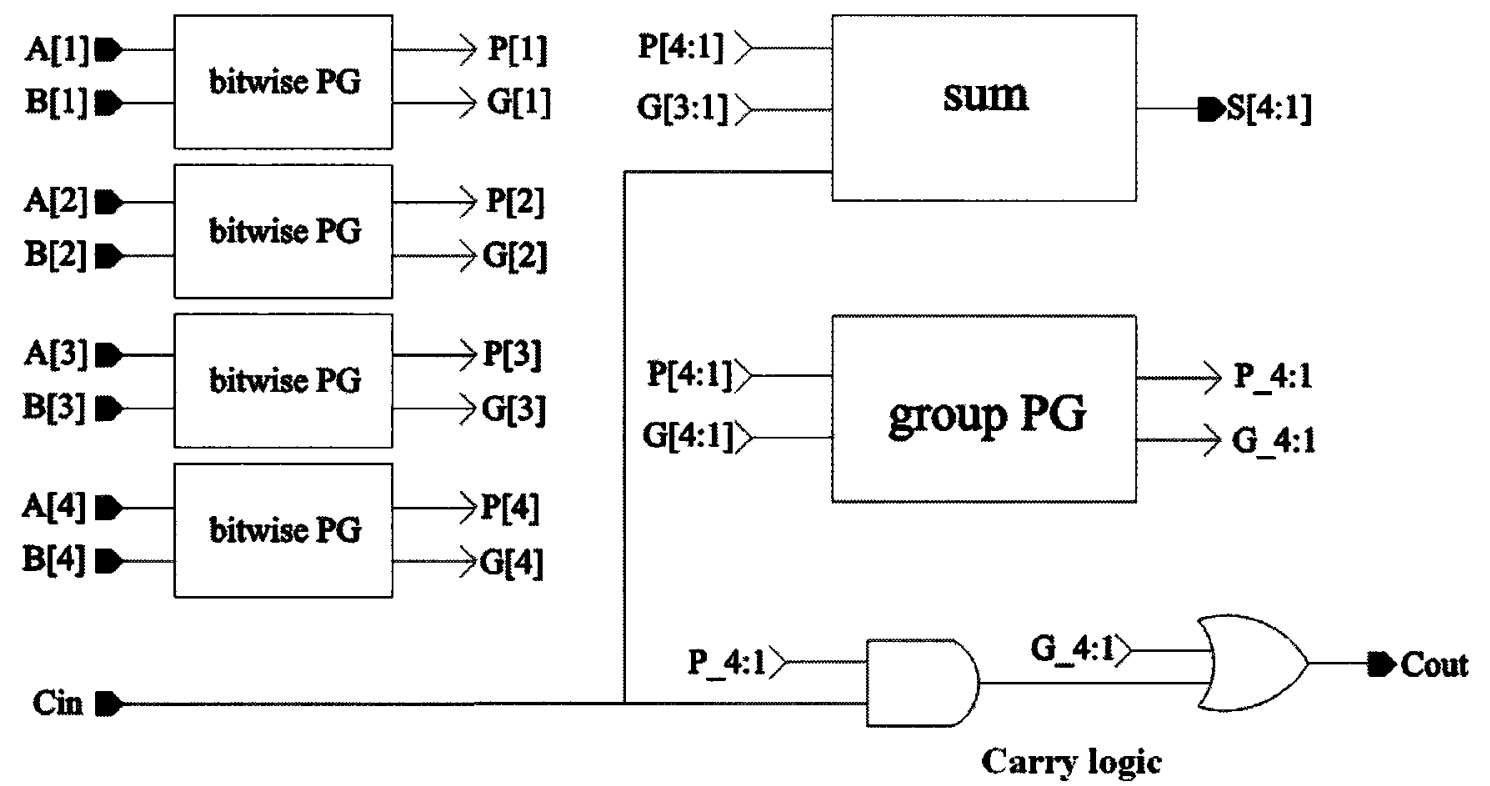

Figure 31: Valency-4 carry-lookahead adder block

Three versions of the carry-lookahead adder were designed, all with the $65 \mathrm{~nm} \mathrm{LP}$ technology. The first was a standard design where the superthreshold optimal beta of 
1.49 is used. This represents the typical superthreshold design. The second circuit was made from parallel transistor stacks composed of transistors of the subthreshold optimum width, which for this technology is $0.12 \mu \mathrm{m}$ for both transistor types. The associated effective beta ratio of 1.35 was used. This design represents the subthreshold design methodology of this thesis. The third circuit was a minimum design where all gates are made from minimum-sized transistors. This design would occupy the least area and also consume the least energy since the capacitance would be the smallest. Note that for the $65 \mathrm{~nm} \mathrm{LP}$ technology, minimum-sized transistors are best for subthreshold, so it is expected that this design will perform favorably.

The carry-lookahead adder will be designed using Logical Effort. Each of the e1ght blocks will be 1dentical. Within a block, each of the paths (bitwise PG, group PG, sum, carry) will be sized as a critical path with the appropriate accounting for load and branching.

The layouts will make use of a standard cell methodology, thus no optimization for area is made. Note that while the standard cell height of the kit ( $2.44 \mu \mathrm{m}$ rail-to-rail) was appropriate for the minimum design, this height had to be extended to $2.655 \mu \mathrm{m}$ for the standard design and $4.15 \mu \mathrm{m}$ for the parallel stacks design. A sample NAND gate from the parallel stacks design with four PMOS transistors in parallel per input and six NMOS stacks in parallel is shown in Figure 32. It is clear that should a library of cells be desıgned for subthreshold logic gates, running the polysilicon gate horizontally must be considered in order to keep the heights of the cells within a limit. 


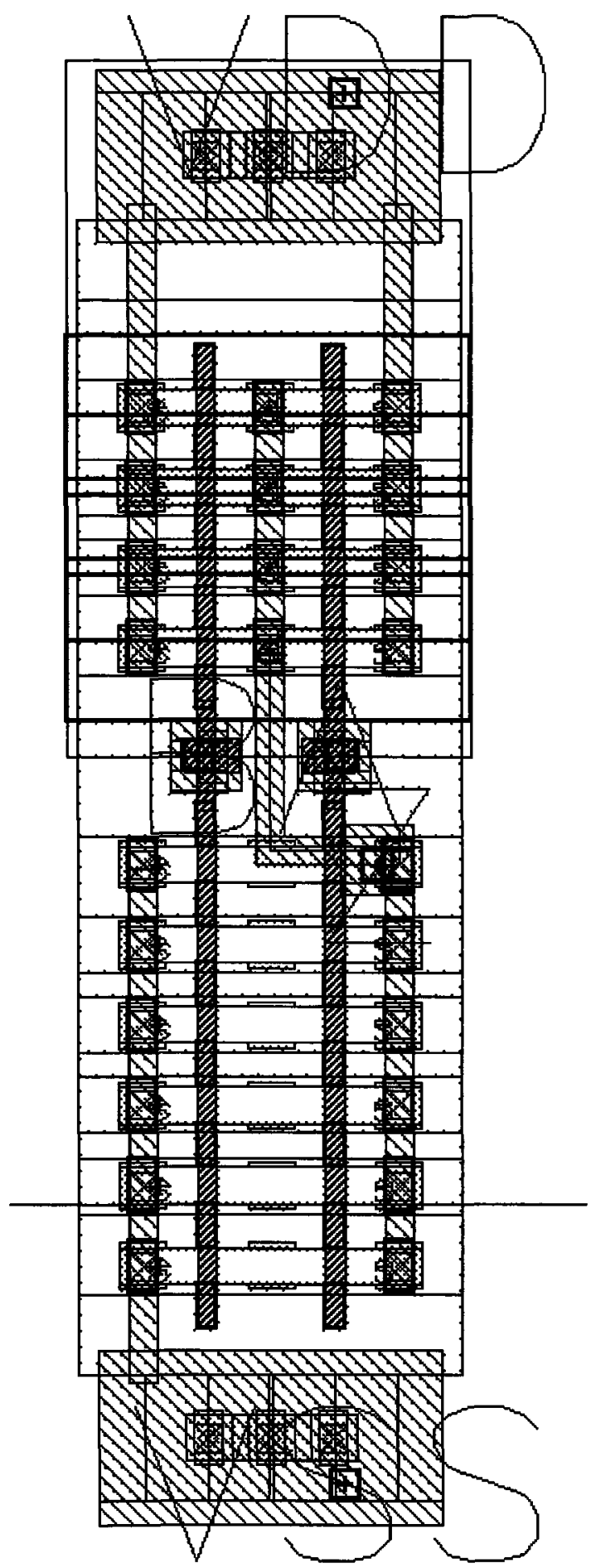

Figure 32: NAND gate from parallel stacks design 
With the exception of the height of the cells, the placement of gates and routing of interconnect was identical for the three designs to provide the fairest comparison of performance. The full layout of the carry-lookahead adder for the standard design is shown in Figure 33. The area is $1997 \mu \mathrm{m}^{2}$. For the minimum design, the area was $1869 \mu \mathrm{m}^{2}$. The parallel stacks design occupied $2887 \mu \mathrm{m}^{2}$. These figures are provided for completeness and should only be used as a rough guide to the relative area requirements of the designs, keeping in mind the caveats from the preceding paragraph.

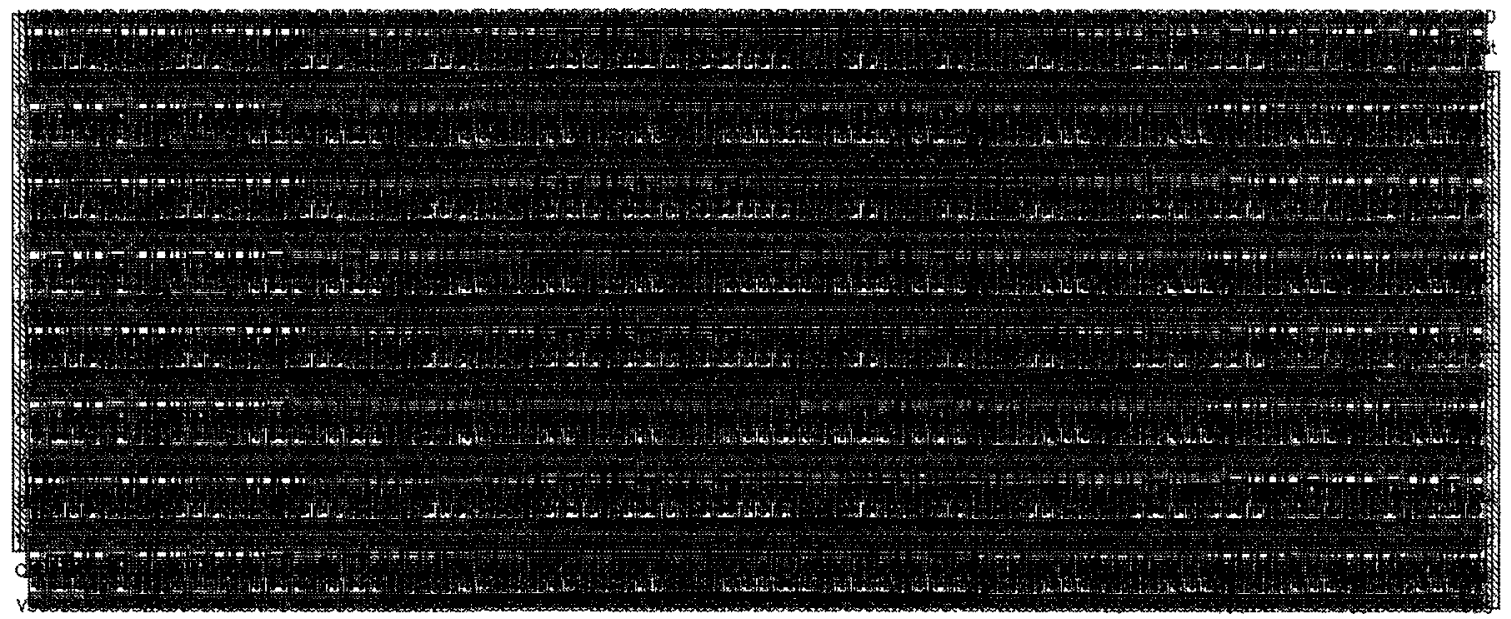

Figure 33: Standard design carry-lookahead adder layout

Schematic and post-layout simulations were done at both 1.2 and $0.3 \mathrm{~V}$. The test cases are described below:

- All bits of $\mathrm{A}, \mathrm{B}$ and $\mathrm{C}_{\mathrm{n}}$ go from 0 to 1 .

- All bits of A, B and $C_{1 n}$ go from 1 to 0 .

- A is fixed at 0 ; all bits of $B$ are fixed at $1 ; C_{1 n}$ goes from 0 to 1 . 
- A is fixed at 0 ; all bits of $B$ are fixed at $1 ; C_{n n}$ goes from 1 to 0 .

- Arbitrary case (a): A goes from 0 to 3642924507 ; B goes from 0 to $1232768243 ; \mathrm{C}_{1 \mathrm{n}}$ goes from 0 to 1.

- Arbitrary case (b): A goes from 0 to 2704529484; B goes from 0 to $3126743191 ; C_{1 n}$ is fixed at 0 .

All inputs are driven through a driver circuit consisting of two inverters whose output rise and fall times are roughly equal. All outputs are loaded with an inverter with PMOS and NMOS widths of 0.24 and $0.12 \mu \mathrm{m}$, respectively.

Table 26 contains the superthreshold results with the post-layout simulation results presented in brackets. Other results besides the propagation delay are reported. The static current with all inputs at 0 and at 1 are shown. The average of these two values will be considered the static current. The energy of operation is found as follows: (i) calculate the total energy for the entire simulation by multiplying the average current by $V_{D D}$ and the simulation time; (ii) find the total static energy by multiplying the static current by $V_{D D}$ and the simulation time; (iii) find the dynamic energy by subtracting the total static energy from the total energy; and (iv) the energy of operation is then the sum of the dynamic energy and the static energy for the time of operation, found by multiplying the static current by $V_{D D}$ and the propagation delay. The energy-delay product, a measure of efficiency, is found by multiplying the energy of operation by the propagation delay. 
Table 26: $65 \mathrm{~nm} \mathrm{LP,} V_{D D}=1.2 \mathrm{~V}$; carry-lookahead adder results (post-layout simulation results in brackets)

\begin{tabular}{|c|c|c|c|c|}
\hline \multicolumn{5}{|c|}{ Standard $1.2 \mathrm{~V}($ sim time $=10 \mathrm{~ns})$} \\
\hline \multicolumn{5}{|c|}{$I_{\text {static }}$ with all inputs $0=43.74(42.69) \mathrm{nA}$} \\
\hline \multicolumn{5}{|c|}{$\mathrm{I}_{\text {static }}$ with all inputs $1=43.32(38.38) \mathrm{nA}$} \\
\hline Test case & $\mathbf{I}_{\mathrm{avg}}[\boldsymbol{\mu} \mathbf{A}]$ & $t_{p}[p s]$ & Energy [fJ]] & EDP $[\mathbf{J} \cdot \mathbf{s}]$ \\
\hline $\mathrm{A}, \mathrm{B}, \mathrm{C} 0 \rightarrow 1$ & $30.47(91.05)$ & $184.5(374.4)$ & $365(1090)$ & $6.74 \mathrm{e}-23(4.09 \mathrm{e}-22)$ \\
\hline $\mathrm{A}, \mathrm{B}, \mathrm{C} 1 \rightarrow 0$ & $45.92(68.88)$ & $174.0(323.3)$ & $551(826)$ & $9.58 \mathrm{e}-23(2.67 \mathrm{e}-22)$ \\
\hline $\begin{array}{l}\mathrm{A}=0, \mathrm{~B}=1, \\
\mathrm{C}, \rightarrow 1\end{array}$ & $20.33(43.41)$ & $889.8(1943.1)$ & $243(521)$ & $2.17 \mathrm{e}-22(1.01 \mathrm{e}-21)$ \\
\hline $\begin{array}{l}\mathrm{A}=0, \mathrm{~B}=1, \\
\mathrm{C} 1 \rightarrow 0\end{array}$ & $23.00(40.96)$ & $934.5(1934.8)$ & $276(491)$ & $2.57 \mathrm{e}-22(9.50 \mathrm{e}-22)$ \\
\hline Case (a) & $23.99(65.63)$ & $262.4(511.8)$ & $287(787)$ & $7.54 e-23(4.03 e-22)$ \\
\hline Case (b) & $26.44(70.82)$ & $338.9(686.3)$ & $317(849)$ & $1.07 e-22(5.83 e-22)$ \\
\hline \multicolumn{5}{|c|}{ Parallel stacks 1.2 V $($ sim time $=10 \mathrm{~ns})$} \\
\hline \multicolumn{5}{|c|}{$\mathrm{I}_{\text {static }}$ with all inputs $0=51.69(56.05) \mathrm{nA}$} \\
\hline \multicolumn{5}{|c|}{$\mathrm{I}_{\text {static }}$ with all inputs $1=52.02(57.86) \mathrm{nA}$} \\
\hline Test case & $\mathbf{I}_{\mathrm{avg}}[\boldsymbol{\mu} \mathbf{A}]$ & $t_{p}[p s]$ & Energy [fJ] & EDP [J·s] \\
\hline $\mathrm{A}, \mathrm{B}, \mathrm{C} 0 \rightarrow 1$ & $35.29(99.97)$ & $189.6(348.5)$ & $423(1200)$ & $8.02 \mathrm{e}-23(4.18 \mathrm{e}-22)$ \\
\hline $\mathrm{A}, \mathrm{B}, \mathrm{C} 1 \rightarrow 0$ & $50.17(76.02)$ & $187.9(318.9)$ & $601(912)$ & $1.13 \mathrm{e}-22(2.91 \mathrm{e}-22)$ \\
\hline $\begin{array}{l}\mathrm{A}=0, \mathrm{~B}=1, \\
\mathrm{C} 0 \rightarrow 1\end{array}$ & $21.96(47.51)$ & $906.7(1880.6)$ & $263(570)$ & $2.38 \mathrm{e}-22(1.07 \mathrm{e}-21)$ \\
\hline $\begin{array}{l}\mathrm{A}=0, \mathrm{~B}=1, \\
\mathrm{C} 1 \rightarrow 0\end{array}$ & $24.27(43.54)$ & $1003.8(1969.2)$ & $291(522)$ & $2.92 \mathrm{e}-22(1.03 \mathrm{e}-21)$ \\
\hline Case (a) & $27.48(72.45)$ & $262.3(478.5)$ & $329(869)$ & $8.63 e-23(4.16 e-22)$ \\
\hline Case (b) & $30.07(77.76)$ & $350.6(677.2)$ & $360(932)$ & $1.26 \mathrm{e}-22(6.31 \mathrm{e}-22)$ \\
\hline \multicolumn{5}{|c|}{ Minimum 1.2 V $($ sim time $=10 \mathrm{~ns})$} \\
\hline \multicolumn{5}{|c|}{$\mathrm{I}_{\text {static }}$ with all inputs $0=30.45(32.85) \mathrm{nA}$} \\
\hline \multicolumn{5}{|c|}{$\mathrm{I}_{\text {static }}$ with all inputs $1=25.68(27.63) \mathrm{nA}$} \\
\hline Test case & $\mathbf{I}_{\mathrm{avg}}[\boldsymbol{\mu} \mathbf{A}]$ & $t_{p}[p s]$ & Energy [fJ] & EDP [J·s] \\
\hline $\mathrm{A}, \mathrm{B}, \mathrm{C} 0 \rightarrow 1$ & $17.42(79.93)$ & $181.5(660.8)$ & $209(959)$ & $3.79 \mathrm{e}-23(6.34 \mathrm{e}-22)$ \\
\hline $\mathrm{A}, \mathrm{B}, \mathrm{C} 1 \rightarrow 0$ & $26.41(52.84)$ & $193.4(519.1)$ & $317(634)$ & $6.12 \mathrm{e}-23(3.29 \mathrm{e}-22)$ \\
\hline $\begin{array}{l}\mathrm{A}=0, \mathrm{~B}=1, \\
\mathrm{C} 0 \rightarrow 1\end{array}$ & $12.81(37.85)$ & $995.9(3543.4)$ & $153(454)$ & $1.53 \mathrm{e}-22(1.61 \mathrm{e}-21)$ \\
\hline $\begin{array}{l}A=0, B=1, \\
C 1 \rightarrow 0\end{array}$ & $15.46(34.51)$ & $1037.8(3000.1)$ & $185(414)$ & $1.92 \mathrm{e}-22(1.24 \mathrm{e}-21)$ \\
\hline Case (a) & $13.36(56.05)$ & $268.9(853.7)$ & $160(672)$ & $4.30 \mathrm{e}-23(5.74 \mathrm{e}-22)$ \\
\hline Case (b) & $14.60(61.02)$ & $352.8(1099.2)$ & $175(732)$ & $6.17 \mathrm{e}-23(8.05 \mathrm{e}-22)$ \\
\hline
\end{tabular}


Considering the schematic simulation results, the standard design was fastest four times, while the parallel stacks and minimum designs were fastest once each. In terms of efficiency, the minimum design swept the results. The post-layout simulation results showed the parallel stacks design as fastest five times with the standard design coming out on top once. In terms of efficiency, the standard design had a clean sweep.

The subthreshold results are shown in Table 27. For the schematic simulations, the parallel stacks design was always fastest while the minimum design was always the most efficient. For the post-layout simulation results, the parallel stacks design was always the fastest and most efficient. Consider the second test case where all input bits fall from 1 to 0 : the parallel stacks design resulted in a $64.5 \%$ reduction in propagation delay compared to the standard design.

The reduction in propagation delay of the parallel stacks design compared to the minimum design is $41.6 \%$. However, for the schematic simulation, the reduction is only $5.8 \%$. It appears that when the parasitic resistances and capacitances are accounted for, the minimum design does not have the adequate current drive to compensate. A better design for all configurations may have been possible if wire capacitance estimates were included during the Logical Effort design, particularly since parasitics play a greater role in the deep submicron technologies. Logical Effort also tends to yield better designs when driving a larger load versus the small inverter considered here. 
Table 27: $65 \mathrm{~nm}$ LP, $V_{D D}=0.3 \mathrm{~V}$; carry-lookahead adder results (post-layout simulation results in brackets)

\begin{tabular}{|c|c|c|c|c|}
\hline \multicolumn{5}{|c|}{ Standard $0.3 \mathrm{~V}(\mathrm{sim}$ time $=10 \mu \mathrm{s})$} \\
\hline \multicolumn{5}{|c|}{$\mathrm{I}_{\text {static }}$ with all inputs $0=8.834(8.853) \mathrm{nA}$} \\
\hline \multicolumn{5}{|c|}{$\mathrm{I}_{\text {static }}$ with all inputs $1=7.690(6.920) \mathrm{nA}$} \\
\hline Test case & $\mathbf{I}_{\text {avg }}[\mathbf{n} \mathbf{A}]$ & $\mathbf{t}_{\mathbf{p}}[\mathbf{n s}]$ & Energy [fJ] & EDP $[\mathbf{J} \cdot \mathbf{s}]$ \\
\hline $\mathrm{A}, \mathrm{B}, \mathrm{C} 0 \rightarrow 1$ & $15.09(28.68)$ & $333.1(1007.0)$ & $21.3(64.8)$ & $7.10 \mathrm{e}-21(6.52 \mathrm{e}-20)$ \\
\hline $\mathrm{A}, \mathrm{B}, \mathrm{C} 1 \rightarrow 0$ & $19.61(25.29)$ & $395.6(869.9)$ & $35.0(54.3)$ & $1.39 \mathrm{e}-20(4.72 \mathrm{e}-20)$ \\
\hline $\begin{array}{l}A=0, B=1, \\
C 0 \rightarrow 1\end{array}$ & $13.65(18.78)$ & $1815.2(4908.5)$ & $20.7(44.3)$ & $3.75 \mathrm{e}-20(2.17 \mathrm{e}-19)$ \\
\hline $\begin{array}{l}\mathrm{A}=0, \mathrm{~B}=1, \\
\mathrm{C} 1 \rightarrow 0\end{array}$ & $14.57(18.41)$ & $1993.8(4605.7)$ & $23.9(42.5)$ & $4.76 \mathrm{e}-20(1.96 \mathrm{e}-19)$ \\
\hline Case (a) & $13.82(23.48)$ & $487.9(1322.7)$ & $17.9(49.9)$ & $8.73 e-21(6.60 e-20)$ \\
\hline Case (b) & $14.42(24.98)$ & $664.0(1629.7)$ & $20.1(55.1)$ & $1.34 \mathrm{e}-20(8.99 \mathrm{e}-20)$ \\
\hline \multicolumn{5}{|c|}{ Parallel stacks $0.3 \mathrm{~V}($ sim time $=10 \mu \mathrm{s})$} \\
\hline \multicolumn{5}{|c|}{$\mathrm{I}_{\text {static }}$ with all inputs $0=13.82(14.92) \mathrm{nA}$} \\
\hline \multicolumn{5}{|c|}{$I_{\text {static }}$ with all inputs $1=13.50(14.97) \mathrm{nA}$} \\
\hline Test case & $\mathbf{I}_{\text {avg }}[\mathbf{n} \mathbf{A}]$ & $t_{p}[n s]$ & Energy [fJ] & EDP $[\mathbf{J} \cdot \mathbf{s}]$ \\
\hline $\mathrm{A}, \mathrm{B}, \mathrm{C} 0 \rightarrow 1$ & $22.08(39.15)$ & $178.2(380.8)$ & $26.0(74.3)$ & $4.63 e-21(2.83 e-20)$ \\
\hline $\mathrm{A}, \mathrm{B}, \mathrm{C} 1 \rightarrow 0$ & $25.63(33.30)$ & $195.2(308.5)$ & $36.7(56.4)$ & $7.17 \mathrm{e}-21(1.74 \mathrm{e}-20)$ \\
\hline $\begin{array}{l}\mathrm{A}=0, \mathrm{~B}=1, \\
\mathrm{C} 0 \rightarrow 1\end{array}$ & $20.45(27.93)$ & $924.9(2078.8)$ & $24.2(48.3)$ & $2.23 \mathrm{e}-20(1.00 \mathrm{e}-19)$ \\
\hline $\begin{array}{l}\mathrm{A}=0, \mathrm{~B}=1, \\
\mathrm{C} 1 \rightarrow 0\end{array}$ & $21.10(26.71)$ & $1004.4(1807.6)$ & $26.4(43.4)$ & $2.66 \mathrm{e}-20(7.84 \mathrm{e}-20)$ \\
\hline Case (a) & $20.44(32.79)$ & $254.2(521.3)$ & $21.4(55.9)$ & $5.44 \mathrm{e}-21(2.91 \mathrm{e}-20)$ \\
\hline Case (b) & $21.27(34.30)$ & $340.2(703.6)$ & $24.2(61.2)$ & $8.24 \mathrm{e}-21(4.31 \mathrm{e}-20)$ \\
\hline \multicolumn{5}{|c|}{ Minimum $0.3 \mathrm{~V}($ sim time $=10 \mu \mathrm{s})$} \\
\hline \multicolumn{5}{|c|}{$\mathrm{I}_{\text {static }}$ with all inputs $0=8.000(8.624) \mathrm{nA}$} \\
\hline \multicolumn{5}{|c|}{$\mathrm{I}_{\text {static }}$ with all inputs $1=6.670(7.170) \mathrm{nA}$} \\
\hline Test case & $\mathbf{I}_{\text {avg }}[\mathbf{n A}]$ & $t_{p}[\mathbf{n s}]$ & Energy [fJ] & EDP [J·s] \\
\hline $\mathrm{A}, \mathrm{B}, \mathrm{C} 0 \rightarrow 1$ & $10.99(26.88)$ & $181.2(799.6)$ & $11.4(58.8)$ & $2.06 \mathrm{e}-21(4.71 \mathrm{e}-20)$ \\
\hline $\mathrm{A}, \mathrm{B}, \mathrm{C} 1 \rightarrow 0$ & $14.24(21.46)$ & $207.3(528.1)$ & $21.2(41.9)$ & $4.39 \mathrm{e}-21(2.21 \mathrm{e}-20)$ \\
\hline $\begin{array}{l}A=0, B=1, \\
C 0 \rightarrow 1\end{array}$ & $10.68(17.31)$ & $1025.5(4092.7)$ & $12.3(37.9)$ & $1.26 \mathrm{e}-20(1.55 \mathrm{e}-19)$ \\
\hline $\begin{array}{l}\mathrm{A}=0, \mathrm{~B}=1, \\
\mathrm{C} 1 \rightarrow 0\end{array}$ & $11.69(16.70)$ & $1084.9(2826.5)$ & $15.5(33.1)$ & $1.68 \mathrm{e}-20(9.36 \mathrm{e}-20)$ \\
\hline Case (a) & $10.48(21.62)$ & $267.4(1021.3)$ & $10.0(43.6)$ & $2.68 \mathrm{e}-21(4.45 \mathrm{e}-20)$ \\
\hline Case (b) & $10.83(22.83)$ & $352.3(1248.4)$ & $11.3(47.8)$ & $3.97 \mathrm{e}-21(5.96 \mathrm{e}-20)$ \\
\hline
\end{tabular}




\section{CHAPTER 6 - CONCLUSIONS AND FUTURE WORK}

The conclusions of this thesis will be described in this chapter. Also, possible future work to expand upon this research will be described.

\subsection{Conclusions}

In subthreshold, the current of a transistor may not increase linearly with its width. In fact, if the transistor exhibits the inverse-narrow-width effect, where the threshold voltage drops as the width narrows, the current's exponential dependence on the threshold voltage can result in surprising increases in current as the width is narrowed. Since a transistor's capacitances still continue to increase linearly with the width, it may be possible to find a width near the minimum size that clearly maximizes the current-to-capacitance ratio of a transistor, giving rise to what we call a subthreshold optimum width.

The subthreshold optimum width is the key component of the design methodology of this thesis to improve the subthreshold performance of logic circuits. Whether a gate requires transistors stacked in series or more current drive, different configurations of transistors locked to the subthreshold optimum width should be used. To complete a design for these cases, further refinements such as the parallel transistor ratio, describing the ideal ratio of PMOS-to-NMOS transistors sized at the subthreshold optimum width, have been developed. The subthreshold optimum widths and the parallel transistor ratio are then combined into an effective beta ratio to use with transistor sizing 
strategies such as Logical Effort to design subthreshold circuits geared for maximum speed.

Technologies where both transistors exhibit the inverse-narrow-width effect such as $65 \mathrm{~nm} \mathrm{LP}$ and GP both showed significant gains in subthreshold performance with the proposed design methodology. Even in the $90 \mathrm{~nm}$ technology, where the PMOS transistor exhibited the narrow-width effect, an increase in threshold voltage with narrowing widths, benefits could be seen from the proposed sizing for subthreshold operation. A 9-inverter ring oscillator, split into parallel transistors, had a 2.63 times increase in frequency and a $49.2 \%$ reduction in energy-delay product, a near doubling of the efficiency, compared to its standard counterpart. Similarly, a ring oscillator composed of nine NAND3 gates with parallel transistor stacks was 2.85 times faster and reduced the energy-delay product by $56.2 \%$. A test circuit, composed of five gates of differing types, demonstrated clear performance improvements using the design methodology. In particular, large load conditions showed the most improvement, in one case resulting in a propagation delay reduction of $61.4 \%$ compared to a standard design. In post-layout simulations of a 32-bit carry-lookahead adder done in the $65 \mathrm{~nm}$ LP kit, a reduction of $64.5 \%$ in propagation delay when compared to a standard superthreshold design was demonstrated.

\subsection{Future Work}

The work performed in this thesis centered on a static CMOS logic style. The benefits of using the subthreshold optimum width with other logic styles such as cascode 
voltage switch logic or pass-transistor could be explored. If work were to be pursued in this direction, consideration to the switching threshold and noise margins, and the overall robustness of the gates, would need to be given since these logic styles are not as robust as static CMOS.

The subthreshold supply voltage was fixed to $0.3 \mathrm{~V}$ due to previous work [17, 18]. However, optimizing this parameter for minimum energy operation could be a topic of further investigation.

The effects of process, temperature and voltage variations could be explored. Comparisons of the degradation in performance in the subthreshold case compared to superthreshold could be examined.

The design methodology developed in this thesis was geared towards performance. Should another measure be the ultimate goal, such as efficiency or least energy, it would be worthwhlle to investigate what modifications to the methodology are necessary to meet those criteria. It has already been shown that the minimum energy operation can fall in the subthreshold region [2]. 


\section{BIBLIOGRAPHY}

[1] S. Fisher, A. Teman, D. Vaysman, A. Gertsman, O. Yadid-Pecht and A. Fish, “Digital Subthreshold Logic Design - Motivation and Challenges," IEEE 25th Convention of Electrical and Electronics Engineers in Israel, IEEEI 2008, pp. 702-706, Dec. 2008.

[2] A. Wang and A. Chandrakasan, "A 180-mV Subthreshold FFT Processor Using a Minimum Energy Design Methodology," IEEE Journal of Solid-State Circuits, vol. 40, no. 1, pp. 310-319, Jan. 2005.

[3] I. E. Sutherland, "Micropipelines," Communications of the ACM, vol. 32, no. 6, pp. 720-738, Jun. 1989.

[4] I. Sutherland and S. Fairbanks, "GasP: A Minimal FIFO Control," Seventh International Symposium on Asynchronous Circuits and Systems, ASYNC 2001, pp. 46-53, Mar. 2001.

[5] L. A. Akers, "The Inverse-Narrow-Width Effect," IEEE Electron Device Letters, vol. 7, no. 7, pp. 419-421, Jul. 1986.

[6] B. Agrawal, V. K. De and J. D. Meindl, "Three-Dimensional Analytical Subthreshold Models for Bulk MOSFET's," IEEE Transactions on Electron Devices, vol. 42, no. 12, pp. 2170-2180, Dec. 1995.

[7] Y. Kim, S. Sridhar and A. Chatterjee, "Trench Isolation Step-Induced (TRISI) Narrow Width Effect on MOSFET," IEEE Electron Device Letters, vol. 23, no. 10, pp. 600-602, Oct. 2002. 
[8] S. S.-S. Chung and T.-C. Li, “An Analytical Threshold-Voltage Model of TrenchIsolated MOS Devices and Nonuniformly Doped Substrates," IEEE Transactions on Electron Devices, vol. 39, no. 3, pp. 614-622, Mar. 1992.

[9] K. K.-L. Hsueh, J. J. Sanchez, T. A. Demassa and L. A. Akers, "Inverse-NarrowWidth Effects and Small-Geometry MOSFET Threshold Voltage Model", IEEE Transactions on Electron Devices, vol. 35, no. 3, pp. 325-338, Mar. 1988.

[10] L. Xinfu, L. Kheeyong, W. Zhihua, X. Zhibin, D. Yongping, N. Hao, W. Yanping, S. Yanping, T. Bin, L. Louis, C. Sally, Y. Xing, H. Feng and S. Yang, “A Study of Inverse Narrow Width Effect of 65nm Low Power CMOS Technology," 9th International Conference on Solid-State and Integrated-Circuit Technology, 2008, ICSICT 2008, pp. 1138-1141, Oct. 2008.

[11] Y. Cheng, T. Sugii, K. Chen, Z. Liu, M.-C. Jeng and C. Hu, "Modeling Reverse Short Channel and Narrow Width Effects in Small Size MOSFET's for Circuit Simulation," 1997 International Conference on Simulation of Semiconductor Processes and Devices, SISPAD '97, pp. 249-252, Sep. 1997.

[12] W. S. Lau, K. S. See, C. W. Eng, W. K. Aw, K. H. Jo, K. C. Tee, J. Y. M. Lee, E. K. B. Quek, H. S. Kim, S. T. H. Chan and L. Chan, “Anomalous Narrow Width Effect in NMOS and PMOS Surface Channel Transistors Using Shallow Trench Isolation," 2005 IEEE Conference on Electron Devices and Solid-State Circuits, pp. 773-776, Dec. 2005.

[13] N. H. E. Weste and D. Harris, CMOS VLSI Design: A Circuits and Systems Perspective, 3rd ed. Toronto: Pearson Addison-Wesley, 2005. 
[14] T. Sakurai and A. R. Newton, "Alpha-Power Law MOSFET Model and its Applications to CMOS Inverter Delay and Other Formulas," IEEE Journal of Solid-State Circuits, vol. 25, no. 2, pp. 584-594, Apr. 1990.

[15] J. M. Rabaey, A. Chandrakasan and B. Nikolic, Digital Integrated Circuits: A Design Perspective, 2nd ed. Toronto: Pearson Education, 2003.

[16] T.-H. Kim, J. Keane, H. Eom and C. H. Kim, "Utilizing Reverse Short-Channel Effect for Optimal Subthreshold Circuit Design," IEEE Transactions on Very Large Scale Integration (VLSI) Systems, vol. 15, no. 7, pp. 821-829, Jul. 2007.

[17] J. Keane, H. Eom, T.-H. Kim, S. Sapatnekar and C. Kim, "Subthreshold Logical Effort: A Systematic Framework for Optimal Subthreshold Device Sizing," 2006 43rd ACM/IEEE Design Automation Conference, pp. 425-428, 2006.

[18] J. Keane, H. Eom, T.-H. Kim, S. Sapatnekar and C. Kim, "Stack Sizing for Optimal Current Drivability in Subthreshold Circuits," IEEE Transactions on Very Large Scale Integration (VLSI) Systems, vol. 16, no. 5, pp. 598-602, May 2008. 